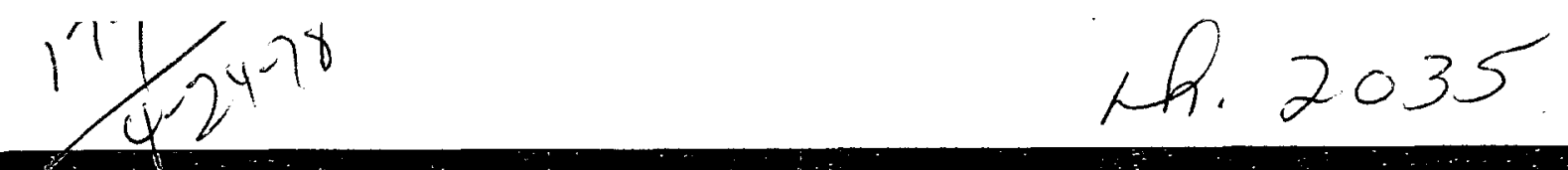

\title{
COCO \\ A COMPUTER PROGRAM \\ FOR SEISMIC ANALYSIS OF A SINGLE COLUMN OF THE HTGR CORE
}

\author{
by \\ N. D. RICKARD
}

Prepared in part under

Contract EY-76-C-03-0167

Project Agreement No. 51

for the San Francisco Operations Office

Department of Energy

DATE PUBLISHED: FEBRUARY 1978

\section{GENERAL ATOMIC COMPANY}




\section{DISCLAIMER}

This report was prepared as an account of work sponsored by an agency of the United States Government. Neither the United States Government nor any agency Thereof, nor any of their employees, makes any warranty, express or implied, or assumes any legal liability or responsibility for the accuracy, completeness, or usefulness of any information, apparatus, product, or process disclosed, or represents that its use would not infringe privately owned rights. Reference herein to any specific commercial product, process, or service by trade name, trademark, manufacturer, or otherwise does not necessarily constitute or imply its endorsement, recommendation, or favoring by the United States Government or any agency thereof. The views and opinions of authors expressed herein do not necessarily state or reflect those of the United States Government or any agency thereof. 


\section{DISCLAIMER}

Portions of this document may be illegible in electronic image products. Images are produced from the best available original document. 
This report was prepared as an account of work sponsored by the United States Government. Neither the United States nor the Department of Energy, nor any of their employees, nor any of their contractors, subcontractors, or their employees, makes any warranty, express or implied, or assumes any legal liability or responsibility for the accuracy, completeness or usefulness of any information, apparatus, product or process disclosed, or represents that its use would not infringe privately owned rights.

Printed in the United States of America Available from

National Technical Information Service

U.S. Department of Commerce

5285 Port Royal Road

Springfield, Virginia 22161

Price: Printed Copy $\$ 5.25$; Microfiche $\$ 3.00$ 
GA-A14600

UC-77

\title{
COCO \\ A COMPUTER PROGRAM \\ FOR SEISMIC ANALYSIS OF A \\ SINGLE COLUMN OF THE HTGR CORE
}

\author{
by \\ N. D. RICKARD
}

This report was prepared as an account of work
sponsored by the United States Government. Neither the
United States nor the United States Department of
Energy, nor any of their employees, nor any of theit
contractors, subcontractors, or their employees, makes
any warranty, express or implied, or assumes any legal
liability or responsibility for the accuracy, completeness
or usefulness of any information, apparatus, product or
process disclosed, or represents that its use would not
infringe privately owned rights.

Prepared in part under

Contract EY-76-C-03-0167

Project Agreement No. 51

for the San Francisco Operations Office

Department of Energy

GENERAL ATOMIC PROJECT 3219

DATE PUBLISHED: FEBRUARY 1978

\section{GENERAL ATOMIC COMPANY}


$\Theta$

$\varepsilon$

. 
ABSTRACT

This document serves as a user's manual and theoretical manual for the COCO code. COCO is a nonlinear numerical integration program designed to analyze a single column of the HTGR core for seismic excitation. Output of the code includes dowel forces, collision forces and a time history of the motion of the blocks. 


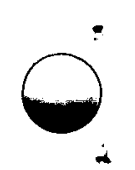

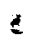

$n$

$\theta$ 
TABLE OF CONTENTS

ABSTRACT . . . . . . . . . . . . . . . . . . . .

1. INTRODUCTION . . . . . . . . . . . . . . . . . . . 1

2. MATHEMAtical MODEL ................... . . . . . . 4

2.1. Sign Convention .................. 4

2.2. Equations of Motion ............... . 4

2.3. Collision Forces . . . . . . . . . . . . . 6

2.4. Dowel Forces . . . . . . . . . . . . . . . . 8

2.5. Friction Forces . . . . . . . . . . . . 12

2.6. Initial Conditions . . . . . . . . . . . . . 12

2.7. Locked Degrees of Freedom . . . . . . . . . . . 14

2.8. PCRV Motion ................. 14

2.8.1. Sinusoidal Dwell . . . . . . . . . 15

2.8.2. Displacement Time History ......... 15

2.8.3. Acceleration Time Histories . . . . . . 16

2.9. Sma11 Angle Approximations . . . . . . . . . . . . 16

3. NUMERICAL INTEGRATION METHODS . . . . . . . . . . . . . 17

3.1. Central Difference . . . . . . . . . . . . . 17

3.2. Second-Order Runge-Kutta . . . . . . . . . . . 18

4. DESCRIPTION OF INPUT . . . . . . . . . . . . . . . . 20

5. DESCRIPTION OF OUTPUT . . . . . . . . . . . . . . 27

5.1. Input Data . . . . . . . . . . . . . . 27

5.2. Time History Output . . . . . . . . . . . 27

5.3. Output Summary . . . . . . . . . . . . . . 28

6. SAMPLE PROBLEM . . . . . . . . . . . . . . . . . . . 29

REFERENCES . . . . . . . . . . . . . . . . . . . 33

APPENDIX A: INPUT DATA FORMS . . . . . . . . . . . . A-1

APPENDIX B: SAMPLE PROBLEM OUTPUT . . . . . . . . . . . . B-1 
1. Typical COCO model . . . . . . . . . . . . . . . 2

2. Coco spring-damper model . . . . . . . . . . . . . . 3

3. Forces acting on a fuel element . . . . . . . . . . . 5

4. Tapered dowel . . . . . . . . . . . . . . . 8

5. Friction force . . . . . . . . . . . . . . . . . 13

6. Sampling problem . . . . . . . . . . . . . . . . 30

7. Block properties . . . . . . . . . . . . . . . 31

TABLES

1. Collision force coordinates . . . . . . . . . . . . . 10

2. Sample problem data . . . . . . . . . . . . . . 32 
SYMBOLS

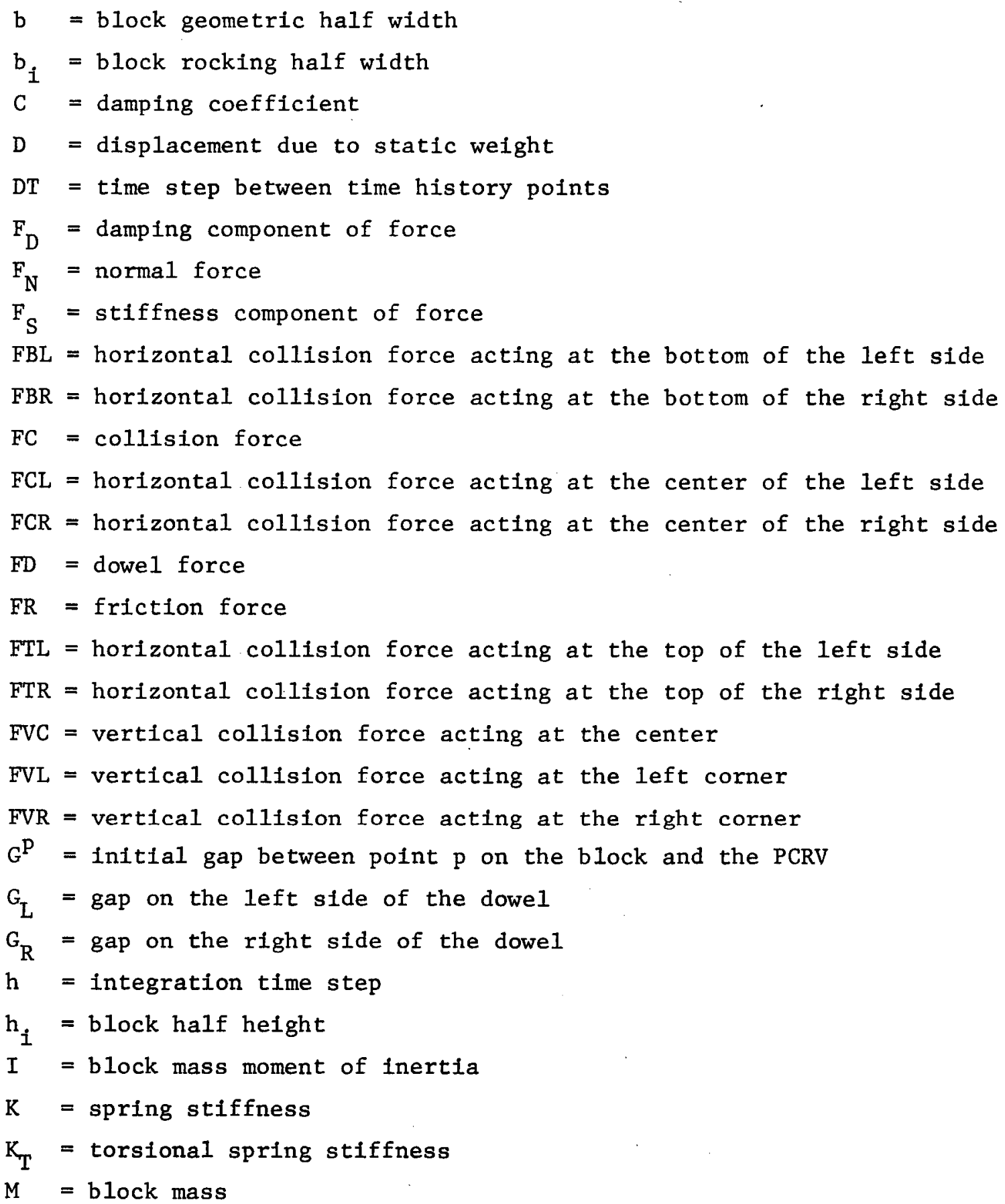




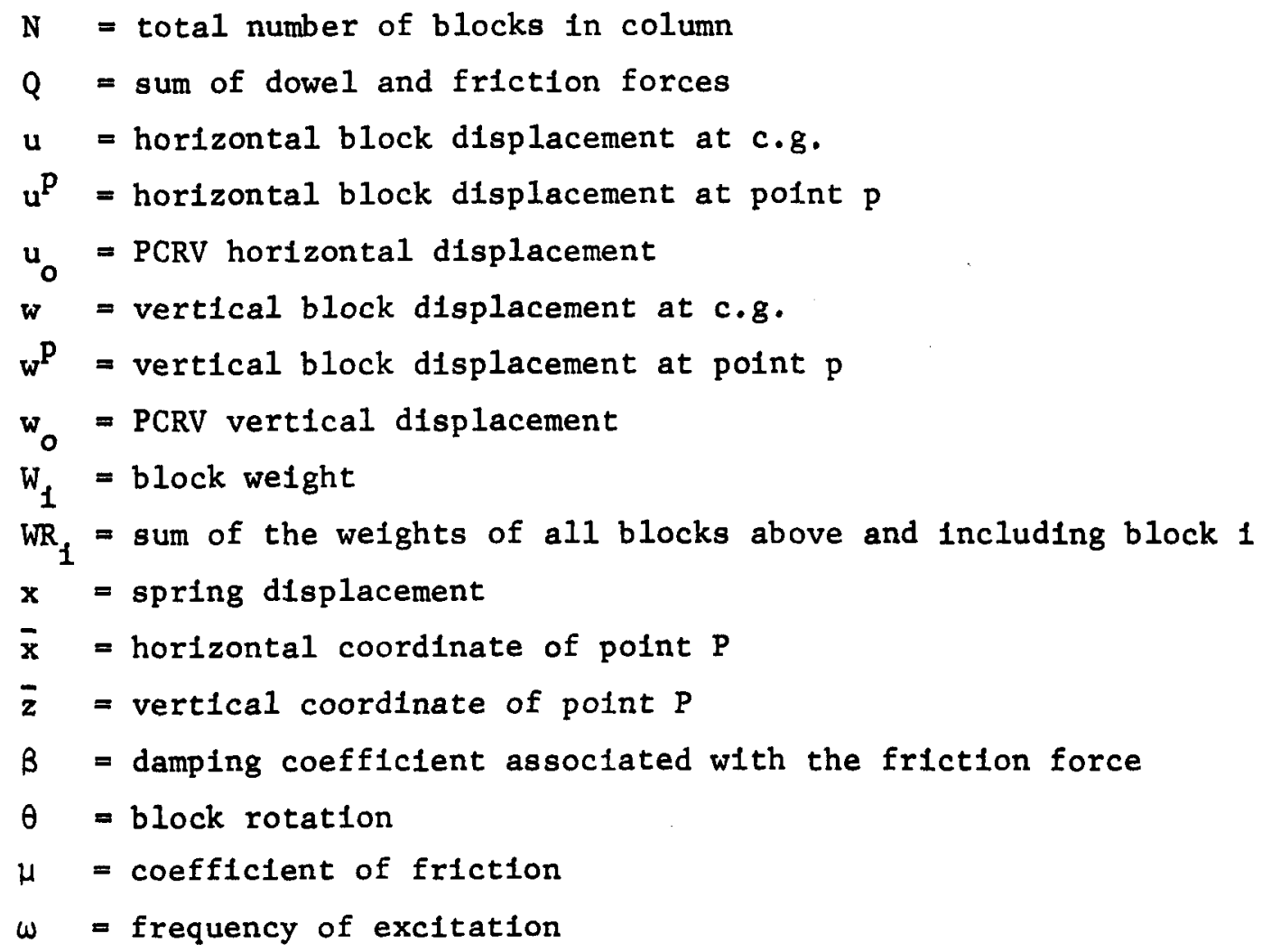




\section{INTRODUCTION}

COCO is designed to analyze single vertical column of the HTGR core constrained within a rigid boundary (Fig. 1). The gaps between the blocks and the boundary are input variables. The column is excited by defining the vertical and horizontal motion of the boundary. The boundary is not permitted to rotate.

Each block is represented by three degrees of freedom; vertical, displacement, horizontal displacement, and rotational about the block's center of gravity. Collision forces are represented by springs and dampers located at the corners and centers of each side of each block (Fig. 2). Dowel forces and friction forces are also computed.

The program has an optional restart capability which, if selected, prints and punches a restart deck. At the termination of each run, a summary of maximum and minimum output values is printed. 


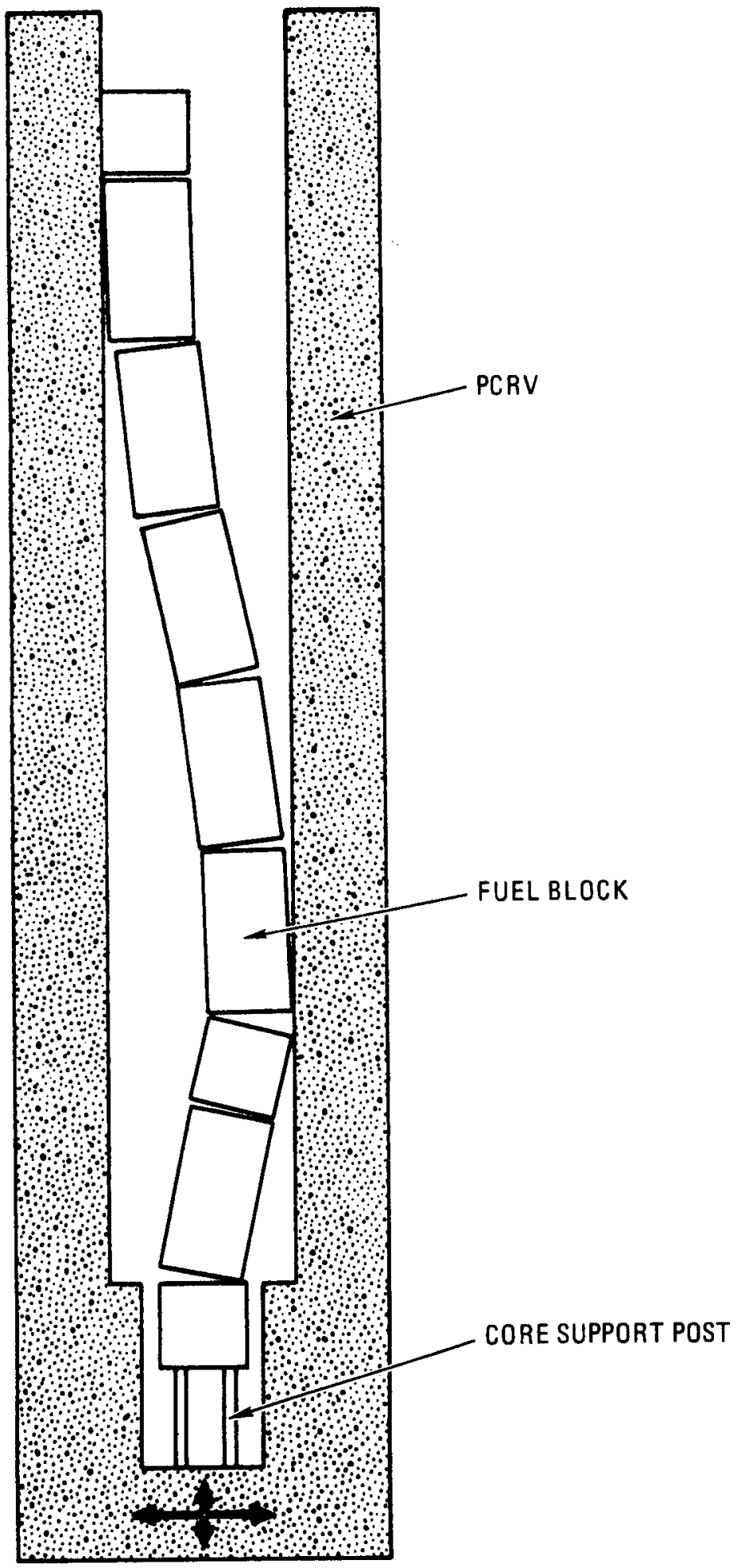

F1g. 1. Typical coco model 


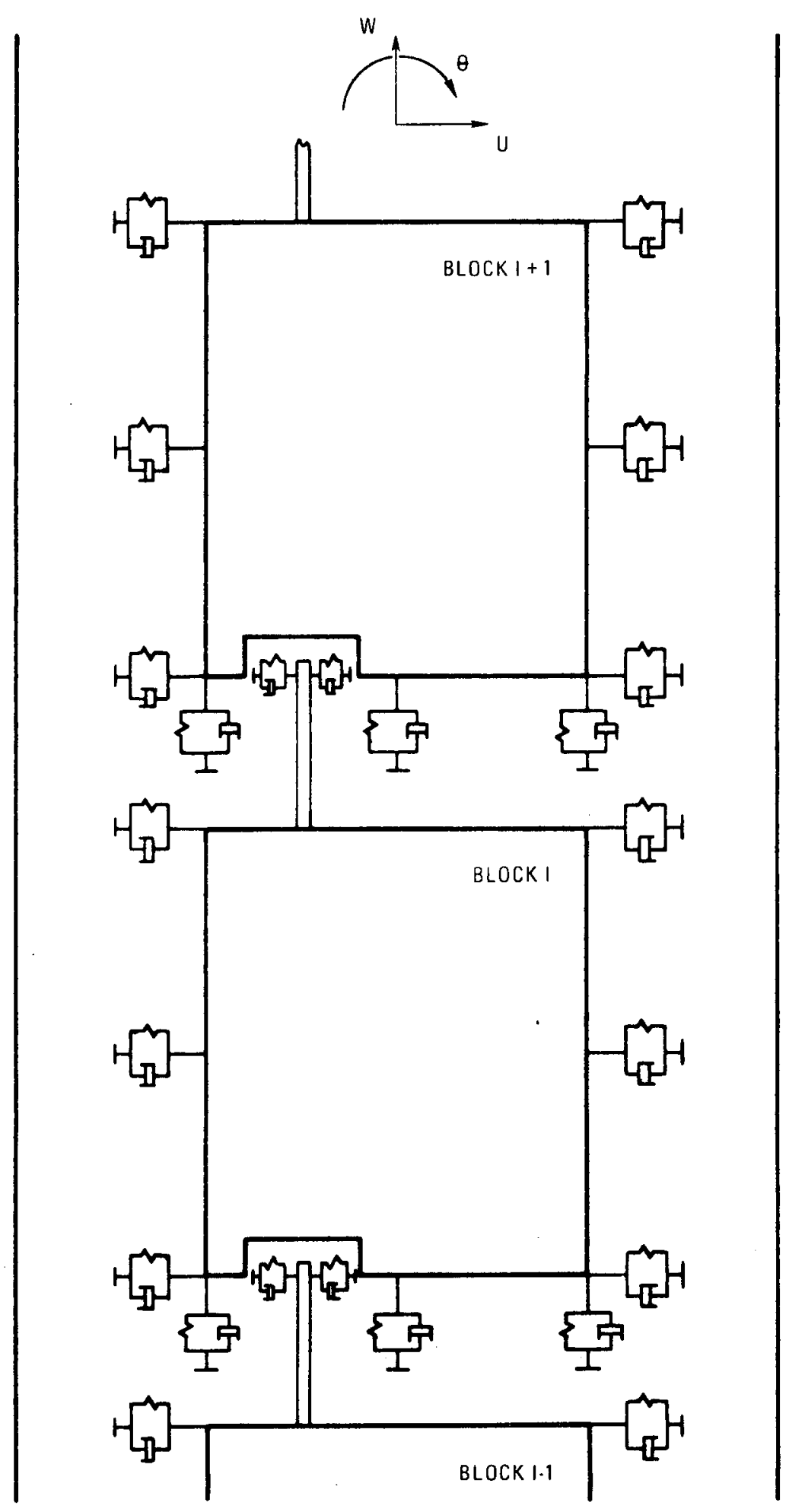

Fig. 2. COCO spring-damper mode1 
2. MATHEMATICAL MODEL

coco models each block as a rigid mass with springs and dampers attached to model the elasticity and damping of the blocks and the connecting dowels. Friction forces are computed at the horizontal interfaces between the blocks.

The following sections discuss in detall the various aspects of the coco mathematical model.

\subsection{SIGN CONVENTION}

The sign convention for the blocks is shown in Fig. 2; where $u$ is positive to the right, $w$ is positive up, and $\theta$ is positive when the top of the block rotates to the right. The sign convention for the PCRV Is the same except that there is no PCRV rotation.

All collision forces are positive. Friction forces and dowel forces acting at the top of a block are positive to the right and at the bottom of a block they are positive to the left.

The blocks are numbered from the bottom up. The PCRV is block 0 .

\subsection{EQUATIONS OF MOTION}

Fig. 3 shows the forces that act upon an individual block. The equations of motion for the block (Ref. 1) are:

$$
m_{1} \ddot{u}_{1}=F T L_{1}+F C L_{1}+F B L_{1}-F T R_{1}-F C R_{1}-F B L_{1}-Q_{1}+Q_{1+1}
$$




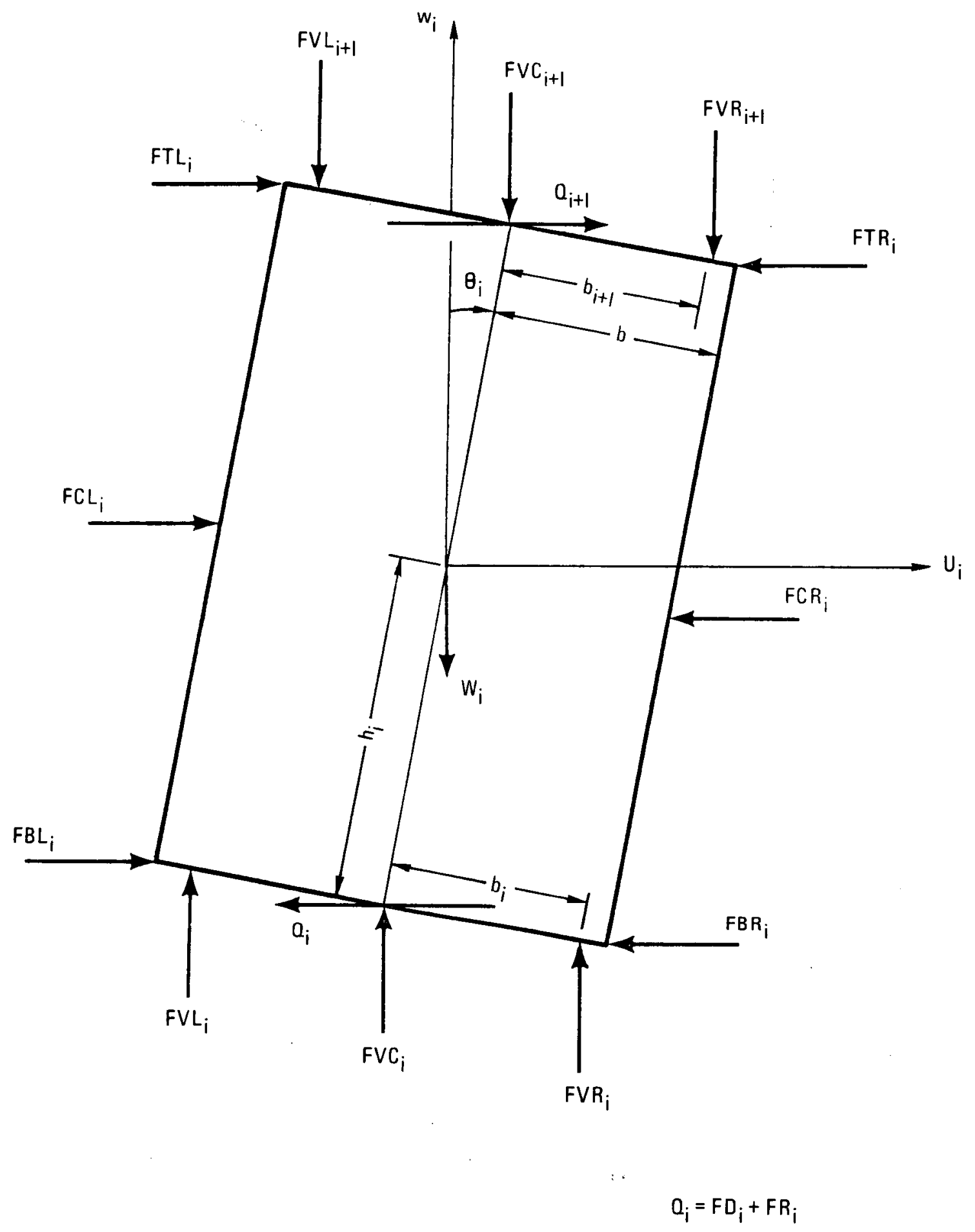

Fig. 3. Forces acting on a fuel element 


$$
\begin{aligned}
& \mathrm{m}_{1} \ddot{\mathrm{w}}_{1}=\mathrm{FVL}_{1}+\mathrm{FVC}_{1}+\mathrm{FVR}_{1}-\mathrm{FVL}_{1+1}-\mathrm{FVC}_{1+1}-\mathrm{FVR}_{1+1}-\mathrm{w}_{1} \\
& I_{1} \ddot{\theta}_{1}=+F V R_{1+1}\left(b_{1+1} \cos \theta_{1}+h_{1} \sin \theta_{1}\right)+F V C_{1+1}\left(h_{1} \sin \theta_{1}\right) \\
& -\mathrm{FVL}_{1+1}\left(\mathrm{~b}_{1+1} \cos \theta_{1}-\mathrm{h}_{1} \sin \theta_{1}\right) \\
& -F V R_{1}\left(b_{1} \cos \theta_{1}-h_{1} \sin \theta_{1}\right)+F V C_{1}\left(h_{1} \sin \theta_{1}\right) \\
& +\mathrm{FVL}_{1}\left(\mathrm{~b}_{1} \cos \theta_{1}+\mathrm{h}_{1} \sin \theta_{1}\right) \\
& -\operatorname{FTR}_{1}\left(h_{1} \cos \theta_{1}-b \sin \theta_{1}\right)+\operatorname{FCR}_{1}\left(b \sin \theta_{1}\right) \\
& +\mathrm{FBR}_{1}\left(\mathrm{~h}_{1} \cos \theta_{1}+b \sin \theta_{1}\right) \\
& -\mathrm{FBL}_{1}\left(h_{1} \cos \theta_{1}-b \sin \theta_{1}\right)+F C L_{1}\left(b \sin \theta_{1}\right) \\
& +\mathrm{FTL}_{1}\left(\mathrm{~h}_{1} \cos \theta_{1}+b \sin \theta_{1}\right)+Q_{1}\left(h_{1} \cos \theta_{1}\right) \\
& +Q_{1+1}\left(h_{1} \cos \theta_{1}\right)
\end{aligned}
$$

Note that $b$ is the geometric half width and $b_{1}$ is the rocking half width.

The bottom block of the column has an optional rotational spring connecting $1 t$ to the PCRV. This spring produces a moment equal to $\mathrm{K}_{\mathrm{T}} \theta_{1}$, which is subtracted from the right side of Eq. 3 for block 1 .

\subsection{COLLISION FORCES}

Colliston forces are modeled at the corners and center of each face of the block. The collision model is a linear spring and damper in series. Forces are computed using the following equations:

$$
F_{C}=F_{S}+F_{D}
$$


where

$$
\begin{aligned}
& \mathrm{F}_{\mathrm{S}}=\mathrm{Kx} \\
& \mathrm{F}_{\mathrm{D}}=\mathrm{C} \dot{\mathrm{x}} .
\end{aligned}
$$

If the deflection of the collision spring $x$ is computed to be less than zero, the collision springs are not in contact and no collision force is calculated.

Collision spring deflections are computed as follows:

right side

$$
x=u_{i}^{p}-G_{i}^{p}-u_{0}
$$

left side

$$
x=u_{0}-u_{i}^{p}-G_{i}^{P}
$$

bottom

$$
x=w_{1-1}^{p}-w_{i}^{p}
$$

The horizontal and vertical translation of each point is evaluated using the following equations (Ref. 2):

$$
\begin{aligned}
& u_{1}^{p}=u_{1}+\bar{z}_{i} \sin \theta_{1}-\bar{x}_{1}\left(1-\cos \theta_{1}\right) \\
& w_{1}^{p}=w_{1}-\bar{x}_{1} \sin \theta_{1}-\bar{z}_{i}(1-\cos \theta) .
\end{aligned}
$$

The equations for computing $\dot{x}$ are generated by differentiating $\mathbf{x}$ with respect to $t$. The results are as follows: 
for the right side,

$$
\dot{x}=\dot{u}_{1}^{p}-\dot{u}_{o}
$$

for the left side,

$$
\dot{x}=\dot{u}_{0}-\dot{u}_{1}^{p}
$$

for the bottom,

$$
\dot{\mathbf{x}}=\dot{\mathrm{w}}_{1-1}^{\mathrm{p}}-\dot{\mathrm{w}}_{1}^{\mathrm{p}}
$$

where

$$
\begin{aligned}
& \dot{u}_{1}^{p}=\dot{u}_{1}+\dot{\theta}_{1}\left(\bar{z} \cos \theta_{1}-\bar{x} \sin \theta_{1}\right) \\
& \dot{w}_{1}^{p}=\dot{w}_{1}-\dot{\theta}_{1}\left(\bar{x} \cos \theta_{1}+z \sin \theta_{1}\right) .
\end{aligned}
$$

Table 1 summarizes the values of $\bar{x}$ and $\bar{z}$ required to compute the various collision forces.

\subsection{DOWEL FORCES}

The dowels are modeled as shown in F1g. 2. Gaps are provided on both the left and right side of the dowel. The dowels are assumed to be tapered such that two blocks can freely rotate even if the dowel gaps are zero (Fig. 4). If cylindrical dowels were used, a block with zero dowel gaps could not rotate.

The following equations define the tapered dowel forces:

$$
F=F_{S}+F_{D}
$$




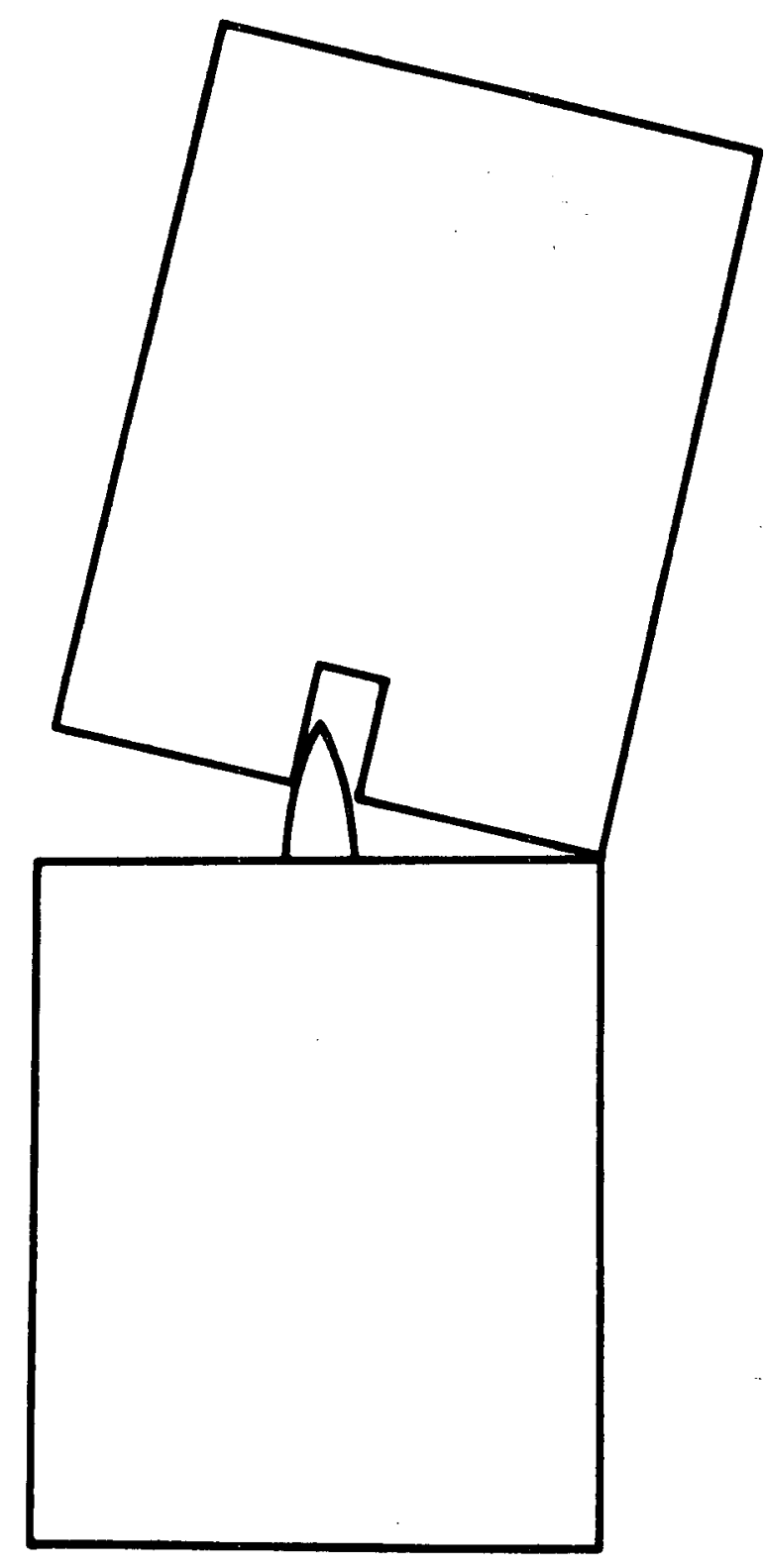

Fig. 4. Tapered dowel

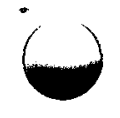

9 
COLLISION FORCE COORDINATES

\begin{tabular}{l|c|c}
\hline Force & $\bar{x}$ & $\bar{z}$ \\
\hline FTL $_{1}$ & $-b$ & $h_{1}$ \\
FCL $_{1}$ & $-b$ & 0 \\
FBL $_{1}$ & $-b$ & $-h_{1}$ \\
FTR $_{1}$ & $b$ & $h_{i}$ \\
FCR $_{1}$ & $b$ & 0 \\
FBR $_{1}$ & $b$ & $-h_{1}$ \\
FVL $_{i}$ & $-b_{1}$ & $-h_{i}$ \\
FVC $_{1}$ & 0 & $-h_{1}$ \\
FVR $_{i}$ & $b_{1}$ & $-h_{1}$ \\
\hline
\end{tabular}


where

$$
\begin{aligned}
& F_{s}=K x \\
& F_{D}=C \dot{x}
\end{aligned}
$$

The deflections are defined as follows: if the top block has a relative displacement to the right,

$$
x=u_{i}^{p}-u_{i-1}^{p}-G_{L}+b_{i}\left(\cos \theta_{i}-\cos \theta_{i-1}\right)
$$

If $x<0$, then $x$ is set to zero, and for a relative displacement to the left

$$
x=u_{i}^{p}-u_{i-1}+G_{R}-b_{i}\left(\cos \theta_{i}-\cos \theta_{i-1}\right)
$$

if $x>0$, then $x$ is set to zero. The $b_{1}$ terms occur due to the model having tapered dowels.

If the dowel deflection is computed to be greater than zero, the dowel damping force is computed using the following value for relative velocity

$$
\dot{x}=\dot{u}_{1}^{p}-\dot{u}_{1-1}^{p}
$$

The terms associated with the tapered dowels have been dropped from Eq. 22 . The velocities $\dot{u}_{i}^{p}$ and $\dot{u}_{i-1}^{p}$ are evaluated by using the following coordinates in Eq. 15

$$
\begin{aligned}
& \bar{z}_{1}=-h_{i} \\
& \bar{z}_{i-1}=h_{i-1} \\
& \bar{x}_{i}=\bar{x}_{i-1}=0 .
\end{aligned}
$$


Substituting these values into Eq. 22 yields

$$
\dot{x}=\dot{u}_{i}-\dot{\theta}_{i} h_{i} \cos \theta_{i}-\dot{u}_{i-1}-\dot{\theta}_{i-1} h_{i-1} \cos \theta_{i-1} .
$$

\subsection{FRICTION FORCES}

Friction forces are computed at the horizontal interfaces between the blocks. The friction force is computed to be the minimum of the following expressions

$$
F R_{1}=\mu\left(F V L_{1}+F V C_{1}+F V R_{i}\right) \operatorname{sign}(\dot{x})
$$

or

$$
\mathrm{FR}_{1}=\dot{\beta \dot{x}}
$$

where $\dot{x}$ is as defined by Eq. 16 and $\mu$ and $\beta$ are input values. If only the first expression were used to define $F R$, the friction force as a function of velocity would be as shown in Fig. 5a. The discontinuity of FR at $\dot{x}=0$ can be a major numerical integration problem. By defining the friction force to be the minimum value defined by the two equations, the discontinuity of the friction force is eliminated as shown in Fig. 5b.

\subsection{INITIAL CONDITIONS}

The code automatically computes the inftial conditions required for static equilibrium. The equilibrium position is determined by letting the blocks settle to a position such that the vertical spring forces at a surface fust balance the static weight of the blocks above the surface. The code automatically computes this position and sets the inftial vertical displacements.

In addition, the code will compute the initial conditions for the case where the blocks have inftlal angular displacements. The following equations are used to compute these Initial displacements: 


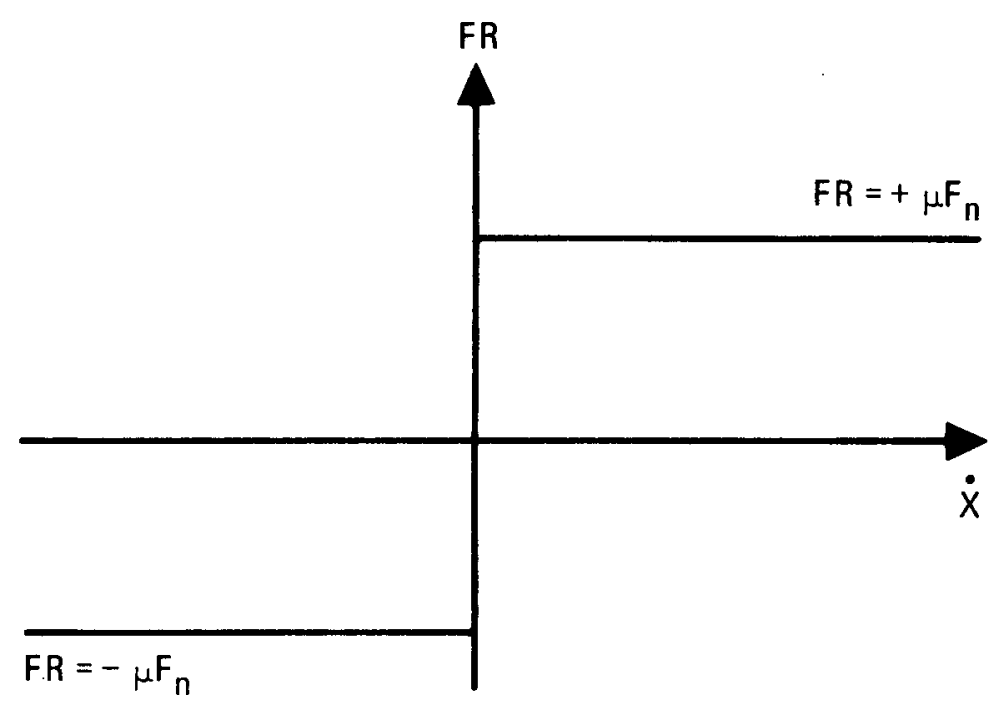

A) FRICTION FORCE AS DEFINED BY E0. 17a

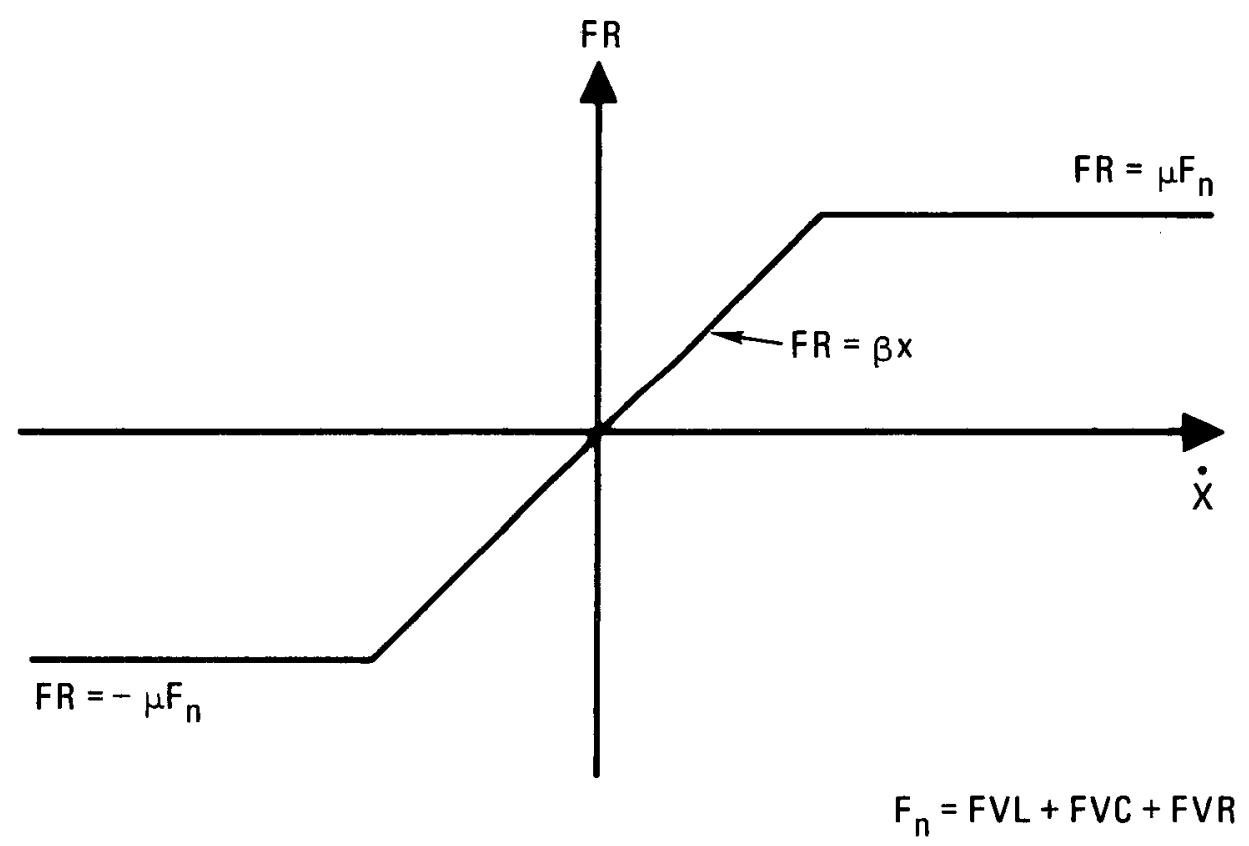

B) FRICTION FORCE AS DEFINED BY THE MINIMUM OF EOS. 17a AND 17b

Fig. 5. Friction force 


$$
\begin{aligned}
w_{i} & =w_{i-1}+\operatorname{sign}\left(\theta_{i}-\theta_{i-1}\right)\left\{b_{i} \sin \theta_{i}-b_{i-1} \sin \theta_{i-1}\right\} \\
& -h_{i}\left(1-\cos \theta_{i}\right)-h_{i-1}\left(1-\cos \theta_{i-1}\right)-D_{i}
\end{aligned}
$$

where if abs $\left(\theta_{i}-\theta_{i-1}\right)<0.002$ radians

$$
D_{i}=W_{i} /\left(2 \operatorname{VCRNS}_{i}+\operatorname{VCNTS}_{i}\right)
$$

or if abs $\left(\theta_{1}-\theta_{1-1}\right) \geq 0.002$ radians

$$
\begin{aligned}
\mathrm{D}_{i} & =\mathrm{WR}_{i} /\left(\mathrm{VCRNS}_{i}\right) \\
\mathrm{u}_{1} & =u_{i-1}+\mathrm{h}_{i} \sin \theta_{i}+\mathrm{h}_{i-1} \sin \theta_{i-1} \\
& +\mathrm{b}_{i}\left(\cos \theta_{i-1}-\cos \theta_{i}\right) \\
\mathrm{WR}_{i} & =\sum_{k=1}^{N} \mathrm{~W}_{k}
\end{aligned}
$$

where $\mathrm{N}$ is the total number of blocks in the column.

\subsection{LOCKED DEGREES OF FREEDOM}

The code has the option to lock any degree of freedom to that of the PCRV. This is done at each step of the integration procedure. The rotational degrees of freedom are set to zero (in COCO the PCRV has no rotational degree of freedom). The vertical degrees of freedom are set to $w_{o}$ and $\dot{w}_{o}$, and the horizontal degrees of freedom are set to $u_{o}$ and $\dot{u}_{0}$.

\subsection{PCRV MOTION}

The code allows the PCRV motion to be specified in three different ways; sinusoidal dwells, acceleration time histories and displacement time histories. In each case both vertical and horlzontal motion can be specified. 


\subsubsection{Sinusoidal Dwell}

Motion for sinusoidal dwells are defined by the following equations

$$
\begin{aligned}
& u_{0}=-\frac{\ddot{u}_{0}}{\omega^{2}} \sin \omega t \\
& \dot{u}_{0}=-\frac{\ddot{u}_{0}}{\omega} \cos \omega t \\
& w_{0}=-\frac{\ddot{w}_{0}}{\omega} \sin \omega t \\
& \dot{w}_{0}=-\frac{\ddot{w}_{0}}{\omega} \cos \omega t
\end{aligned}
$$

\subsubsection{Displacement Time History}

The displacement time histories are input as tables of equally spaced ground displacements. The code automatically computes tables of ground velocities using the following relationships

$$
\begin{aligned}
& \dot{\mathrm{u}}_{\mathrm{o}_{j+1}}=\left(\mathrm{u}_{o_{j+1}}-\mathrm{u}_{\mathrm{o}_{j}}\right) / \mathrm{DT} \\
& \dot{\mathrm{w}}_{\mathrm{o}_{j+1}}=\left(w_{o_{j+1}}-\mathrm{w}_{\mathrm{o}_{j}}\right) / \mathrm{DT} \\
& \dot{\mathrm{u}}_{\mathrm{o}_{1}}=\dot{\mathrm{w}}_{\mathrm{o}_{1}}=0 .
\end{aligned}
$$

The code interpolates from these tables to determine the ground displacements and velocities. 


\subsubsection{Acceleration Time Histories}

Acceleration time histories are input as tables of equally spaced acceleration points. The code interpolates from these tables to determine the acceleration at any time. The velocities and displacements are determined by using the following second-order Runge-Kutta equations to integrate the acceleration input.

$$
\begin{aligned}
& u_{o_{j+1}}=u_{o_{j}}+h \dot{u}_{o_{j}}+\frac{h^{2}}{6}\left(2 \ddot{u}_{o_{j}}+\ddot{u}_{o_{j+1}}\right) \\
& \dot{u}_{o_{j+1}}=\dot{u}_{o_{j}}+\frac{h}{2}\left(\ddot{u}_{o_{j}}+\ddot{u}_{o_{j+1}}\right)
\end{aligned}
$$

The equations for determining $w_{0}$ and $\dot{w}_{0}$ are identical to those for $u_{0}$ and $\dot{u}_{0}$.

\subsection{SMALL ANGLE APPROXIMATIONS}

The following small angle approximations were made in the code:

$$
\begin{aligned}
& \cos \theta \cong 1 \\
& \sin \theta \cong \theta \\
& 1-\cos \theta \cong 1 / 2 \theta^{2} \\
& \cos \theta_{1}-\cos \theta_{i+1} \cong 1 / 2\left(\theta_{i+1}^{2}-\theta_{i}^{2}\right) .
\end{aligned}
$$




\section{NUMERICAL INTEGRATION METHODS}

Two integration methods are available in COCO; central difference and second-order Runge-Kutta. The results of the study presented in Ref. 3 indicate that for COCO the central difference method is the most economical.

\subsection{CENTRAL DIFFERENCE}

The central difference method which is used in COCO is based on the central difference method presented in Ref. 4. This method uses the following equations:

$$
\begin{aligned}
& \dot{x}_{n+1 / 2}=\dot{x}_{n-1 / 2}+h \ddot{x}_{n} \\
& x_{n+1}=x_{n}+h \dot{x}_{n+1 / 2}
\end{aligned}
$$

where

$$
\ddot{x}_{n}=f\left(x_{n}, \dot{x}_{n-1 / 2}, t_{n}\right)
$$

The method is not self-starting since one past value of $\dot{x}$ is required. It is started in the code by using the following equation which is based on Euler's method:

$$
\dot{x}_{n+1 / 2}=\dot{x}_{n}+\frac{h}{2} \ddot{x}_{n}
$$

Stability of the central difference method for linear systems requires that 


$$
\mathrm{h}<\frac{2}{\omega_{\max }}
$$

where $\omega_{\max }=$ highest linear frequency of the system.

Reference 4 states "there is considerable empirical evidence that these stability limits are valid for nonlinear problems if the current highest frequency .... Is used in the equation."

\subsection{SECOND-ORDER RUNGE-KUTTA}

The second-order Runge-Kutta method, which is used, was taken from Ref. 5, Eq. 3.12. The values selected for the constants are

$$
\begin{aligned}
& \gamma_{1}=\gamma_{2}=1 / 2 \\
& \alpha_{1}=\alpha_{2}=2 / 3 \\
& \alpha_{3}=4 / 3 \\
& \sigma_{1}=1 / 2 \\
& \sigma_{2}=3 / 2 .
\end{aligned}
$$

The selection of these constants causes the method to use the acceleration at $t=t_{0}$ and $t_{0}+2 / 3 \mathrm{~h}$ to evaluate the displacements and velocities at $t=t_{0}+h$.

The following equations are used in the code:

$$
\begin{aligned}
& \ddot{x}_{n}=f\left(t_{n}, x_{n}, \dot{x}_{n}\right) \\
& x_{n+2 / 3}=x_{n}+2 / 3 h \dot{x}_{n}+1 / 3 h^{2} \ddot{x}_{n}
\end{aligned}
$$




$$
\begin{aligned}
& \dot{x}_{n+2 / 3}=\dot{x}_{n}+2 / 3 h \ddot{x}_{n} \\
& t_{n+2 / 3}=t_{n}+2 / 3 h \\
& \ddot{x}_{n+2 / 3}=f\left(t_{n+2 / 3}, x_{n+2 / 3}, \dot{x}_{n+2 / 3}\right) \\
& t_{n+1}=t_{n}+h \\
& x_{n+1}=x_{n}+h \dot{x}_{n}+\frac{h^{2}}{4}\left(\ddot{x}_{n}+\ddot{x}_{n+2 / 3}\right) \\
& \dot{x}_{n+1}=\dot{x}_{n}+\frac{h}{4}\left(\ddot{x}_{n}+3 \ddot{x}_{n+2 / 3}\right) .
\end{aligned}
$$

This method is self-starting. 


\section{DESCRIPTION OF INPUT}

This section describes the input required by coco. The data is entered in either A, F or I (standard FORTRAN). Data read in A format can be any combination of alpha-numeric characters (e.g., ABC1234*ETC). F format data is numeric and requires a decimal point. It can be located anywhere in the data field except when entered in exponential form (e.g., 7.15E-6). In this case, the number should be entered in the columns furthest to the right. I format data is numeric, cannot have a decimal, and must be entered in the columns furthest to the right. Input data forms are presented in Appendix A.

CARD 1

TITLE - any 72 character title

\section{CARD 2}

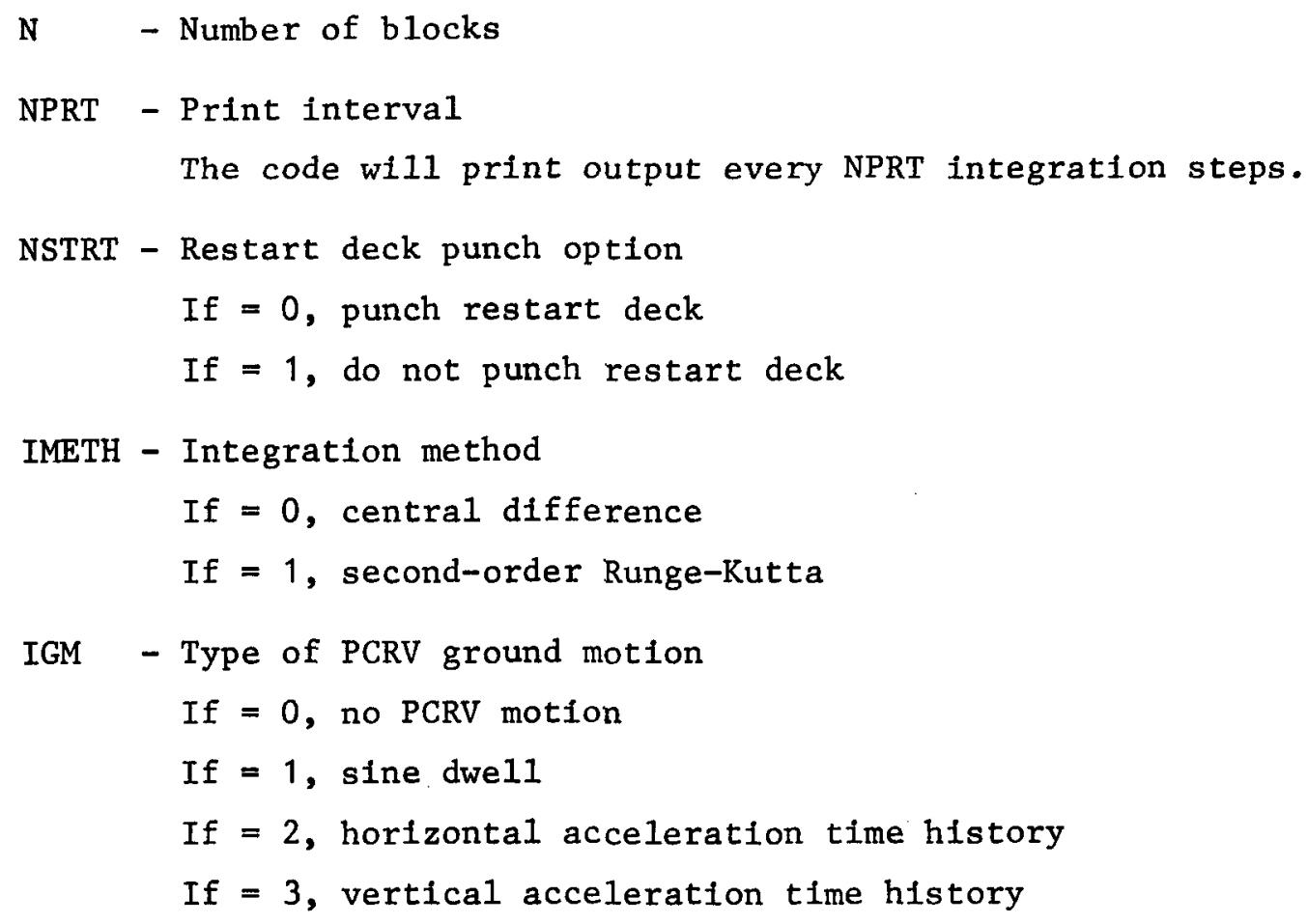




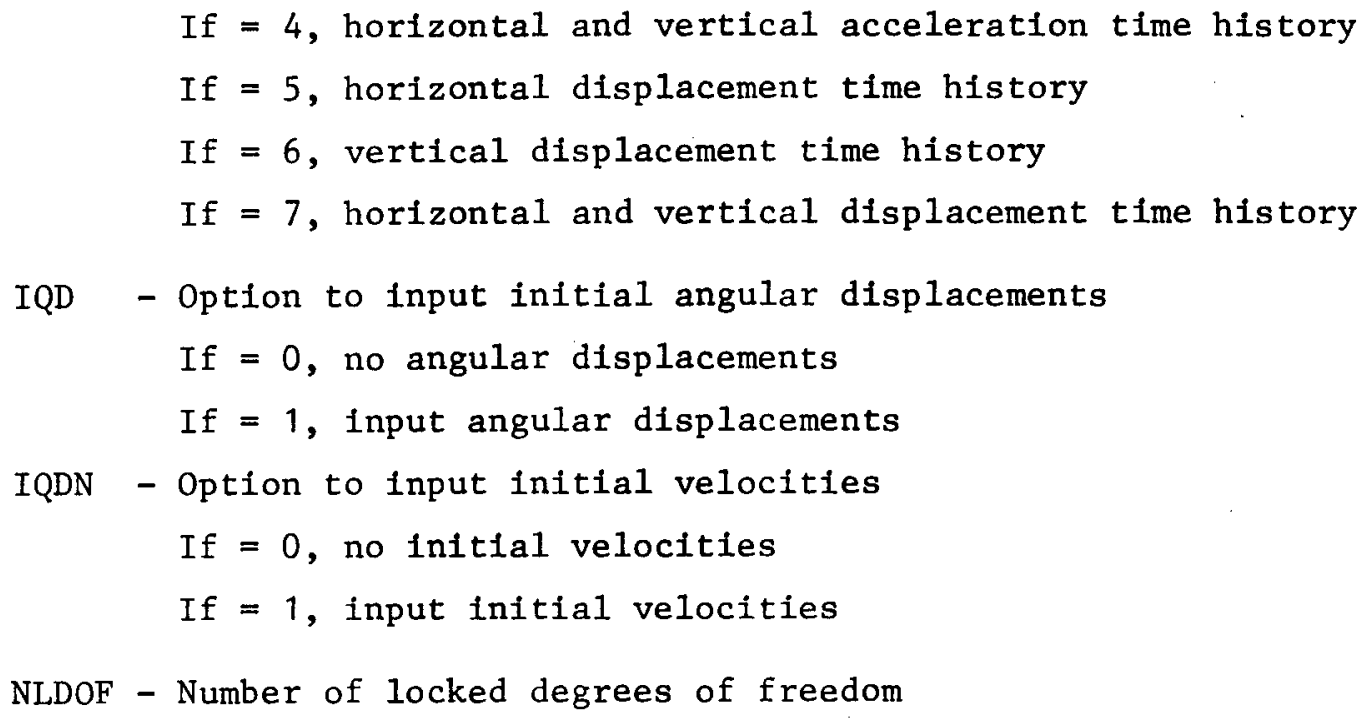

\section{$\underline{\text { CARD } 3}$}

LBLK(I) - Block number of the $I^{\text {th }}$ locked degree of freedom

LCOMP(I) - Component of the Ith locked degree of freedom

$$
\begin{aligned}
& \text { If }=1, \theta \\
& \text { If }=2, u \\
& \text { If }=3, w
\end{aligned}
$$

\section{$\underline{\text { CARD } 4}$}

$$
\begin{aligned}
& \text { TO - Starting time } \\
& \text { TEND - Ending time } \\
& \text { HH - Integration time step }
\end{aligned}
$$

\section{CARD 5}

BG

- Element geometric width

BG is used to compute $b(b=1 / 2 B G)$, which 1 tern is used as a moment arm in the equations of motion and is used to compute collision spring deflections. In addition, $b$ is used in the following equation to compute the mass moment of inertia of the blocks:

$$
I_{i}=1 / 3\left(b^{2}+h_{i}^{2}\right) M_{i}
$$




$$
\begin{aligned}
& \text { RSPRING - Rotational spring stiffness for the bottom block } \\
& \text { UO - Initial PCRV horizontal displacement } \\
& \text { UOD - Initial PCRV horizontal velocity } \\
& \text { Wo - Initial PCRV vertical displacement } \\
& \text { WOD - Initial PCRV vertical velocity }
\end{aligned}
$$

\section{CARD 6}

VCRNS(I) - Vertical corner spring stiffness for block I VCRND(I) - Vertical corner spring damping for block I VCENS(I) - Vertical center spring stiffness for block I VCEND(I) - Vertical center spring damping for block I HCRNS(I) - Horizontal corner spring stiffness for block I HCRND(I) - Horizontal corner spring damping for block I HCENS(I) - Horizontal center spring stiffness for block I HCEND(I) - Horizontal center spring damping for block I

CARD 7

$\operatorname{GAP}(3, \mathrm{I})^{*}$ - Horizontal gap between the PCRV and the upper left corner of block I

\section{CARD 8}

GAP $(4, I)$ - Horizontal gap between the PCRV and the lower left corner of block I

\section{CARD 9}

GAP(1,I) - Horizontal gap between the PCRV and the upper right corner of block I

\section{CARD 10}

GAP(2,I) - Horlzontal gap between the PCRV and the lower right corner of block I

* The center gap is computed as the average of the upper and lower gaps. 
CARD 11

SK - Dowel stiffness

CDW - Dowel damping coefficient

GAP1 - Gap on the right side of the dowe1

GAP2 - Gap on the left side of the dowe1

COF - Block-on-block coefficient of friction

SLP - Viscous damping coefficient for friction force ( $B$ )

\section{CARD 12}

AMPLU - Horizontal g level for sine dwell

FREQU - Horizontal frequency for sine dwe11

AMPLW - Vertical g level for sine dwell

FREQW - Vertical frequency for sine dwe11

\section{CARD 13}

H(I) - Height of block I

$\underline{\text { CARD } 14}$

$B(I)$ - Rocking width of block I

CARD 15

WT(I) - Weight of block I

CARD 16

THETA(I) - Initial angular displacement of block I

CARD 17

THETAD(I) - Inftial angular velocity for block $I$

CARD 18

UD(I) - Initial horizontal velocity for block I 
CARD 19

WD(I) - Initial vertical velocity for block I

CARD 20

NINPU - Number of points in the PCRV horizontal displacement time history table

FACTOR - A factor to convert the input time history to $1.0 \mathrm{~g}$

SCALE - G level of the analysis

DELT - Time step between time history points

CARD 21

GG(1,I) - Point I of the PCRV horfzontal displacement time history table

\section{CARD 22}

NINPUV - Number of points in the vertical displacement time history table

FACTOR - A factor to convert the input time history to $1.0 \mathrm{~g}$

SCALE - G level of the analysis

DELTV - Time step between time history points

DELT on card 20 must be the same as DELTV.

CARD 23

$G G(4, I)$ - Point I of the vertical displacement time history

\section{CARD 24}

NFORMT - Time history input format

If $=0,8 \mathrm{~F} 9.0$

If $=1,8 \mathrm{~F} 10.0$

NINPU - Number of points in the horizontal acceleration time history

FACTOR - A factor to convert the input time history to $1.0 \mathrm{~g}$

SCALE - G level of the analysis

DELT - TIme step between time history points 
CARD 31 (Restart deck)

UO - PCRV horizontal displacement

UOD - PCRV horizontal velocity

WO - PCRV vertical displacement

WOD - PCRV vertical velocity 


\section{DESCRIPTION OF OUTPUT}

Printed output consists of input data, time history results and an output summary. These are discussed in the following sections.

\subsection{INPUT DATA}

Input data is printed in two formats. The first is printed by the input repeat subroutine. This subroutine reads the data and then prints it in exactly the same format as it was read. If the input repeat does not list the complete input deck, then most likely the input deck has a control card error.

Immediately following the input repeat, the program lists the data as interpreted by the code. This data should be checked carefully, since errors in input format (e.g., data entered in the wrong columns or missing decimal points) will be obvious.

In addition to input data, this section also prints some data which was automatically computed by the code.

\subsection{TIME HISTORY OUTPUT}

The main section of the output data is the time history output. This data is printed every NPRT (print interval) integration steps and describes the motion as a function of time. The first line of the time history output is the title. The following is a description of the remaining data:

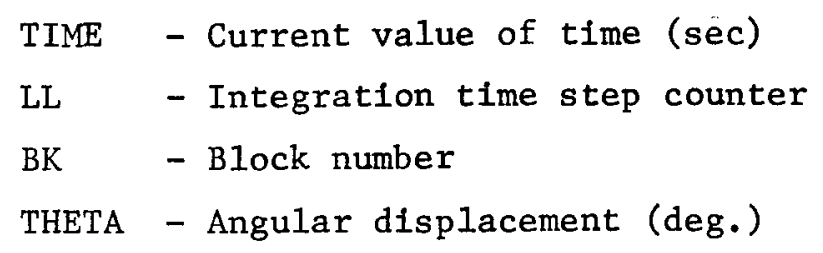




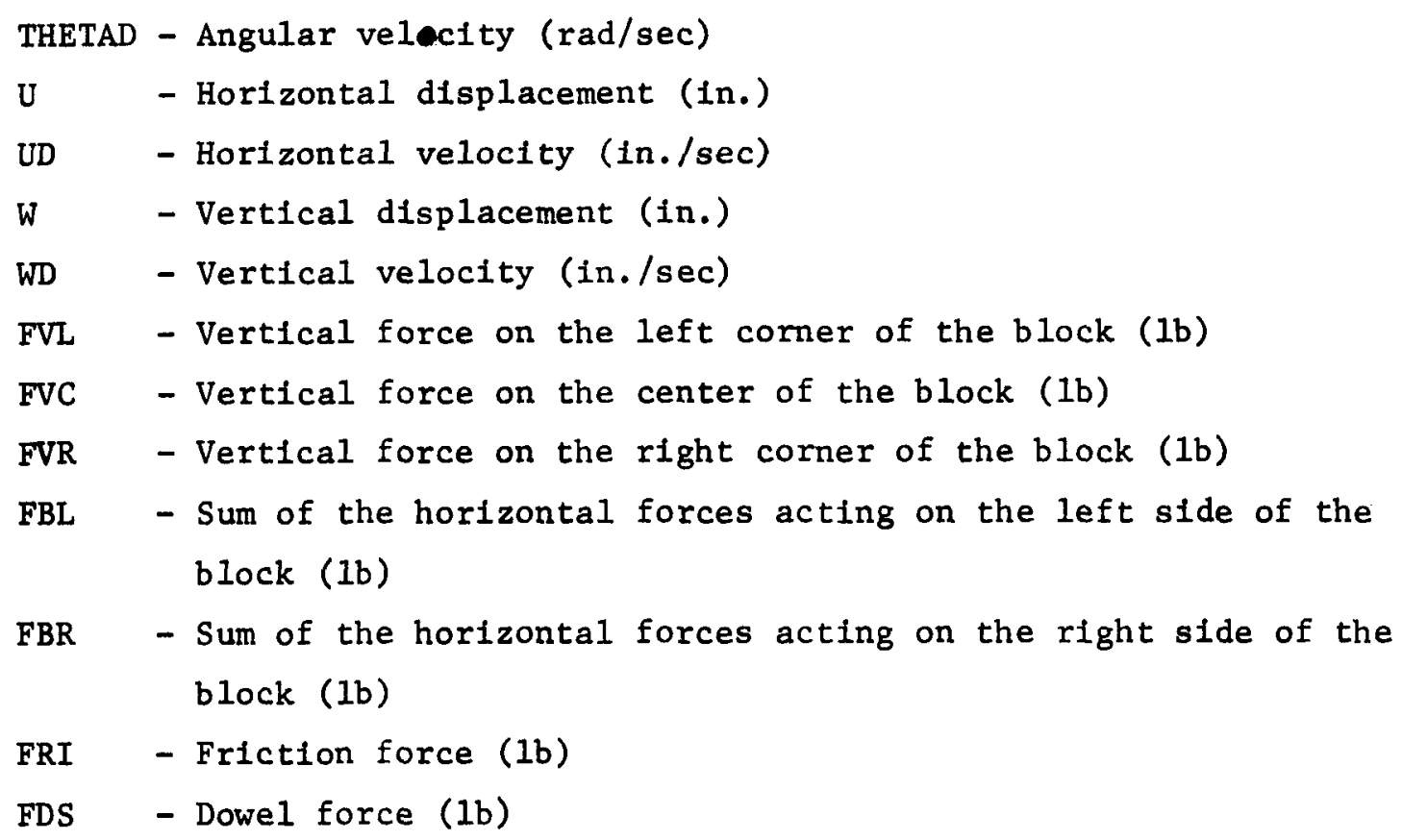

All of the printed forces are the maximum which occurred during the print interval. Only the stiffness portion of the dowel forces and collision forces are printed.

\subsection{OUTPUT SUMMARY}

At the termination of a run, an output summary is printed which gives all of the maximum forces, velocities and displacements along with the times that these maximums occur. 


\section{SAMPLE PROBLEM}

The sample problem consists of five stacked blocks supported on two core support posts as shown in Fig. 6. The model does not represent any real structure. The properties have been selected to minimize computer running time and to illustrate how the program can be used. The current modeling technique is to eliminate the use of the center springs (Ref.6). To make the sample problem more general, the center springs have been included and are assumed to be $40 \%$ of the surface stiffness. The remaining $60 \%$ is divided between the corner springs.

Figure 7 presents the block properties which are used for the analysis. Table 2 presents other sample problem data. Appendix B presents the output from the sample problem analysis. The first page of output is a computer listing of the sample problem input deck.

The following control cards are required to run $\mathrm{COCO}$ :

$$
\begin{array}{ll}
\text { DASG }, \mathrm{X} & \text { COCO*PRODUCTION } \\
\text { DXQT } & \text { COCO*PRODUCTION. ABS }
\end{array}
$$

where $\Delta$ represents a 7-8 punch in column 1. The input deck is placed immediately after the $\nabla X Q T$ card. The sample problem can be automatically run by placing the following card in back of the two card shown above.

VADD COCO*PRODUCTION. SAMPLE 


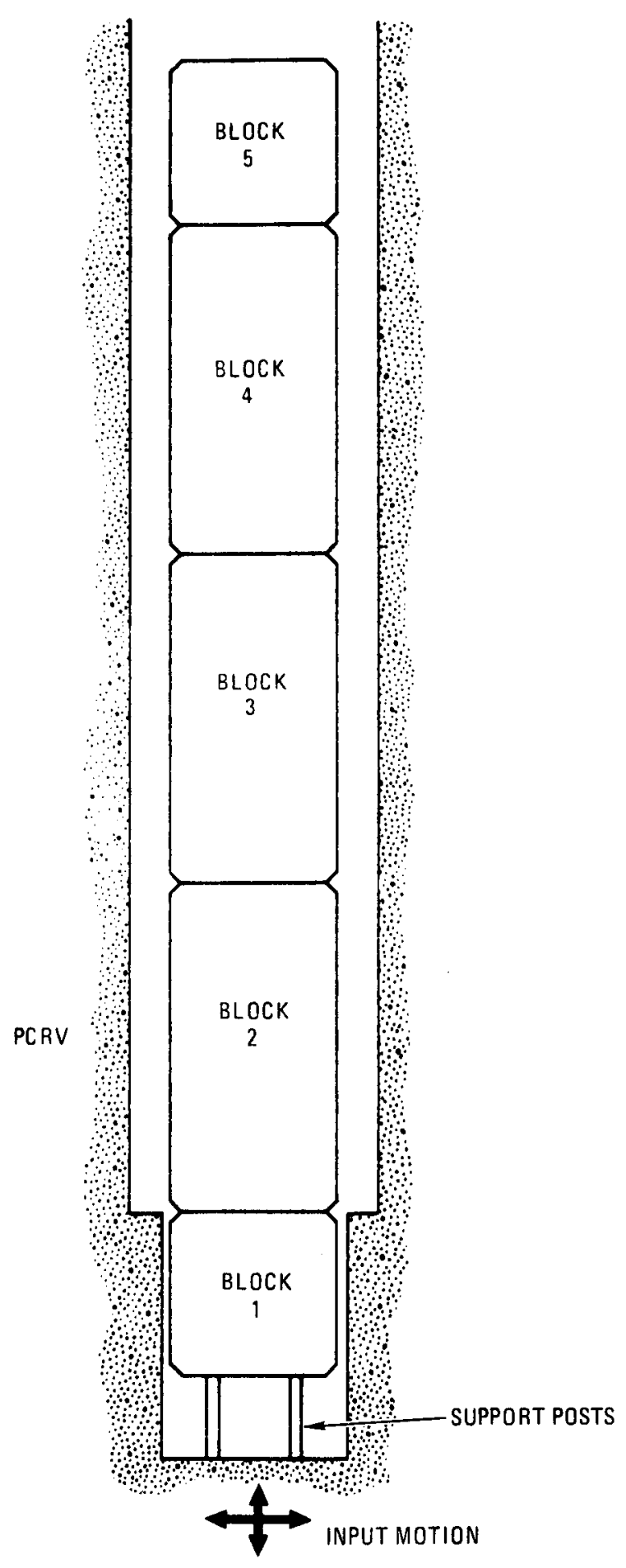

Flg. 6. Sample problem 


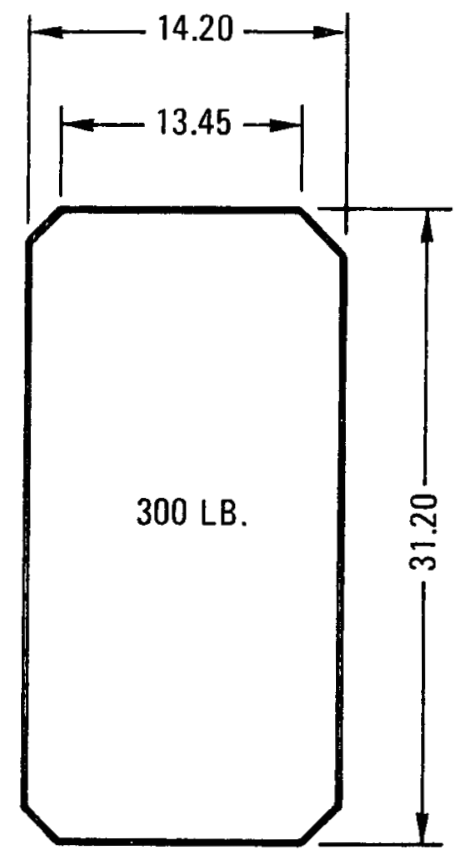

A) FULL HEIGHT BLOCK

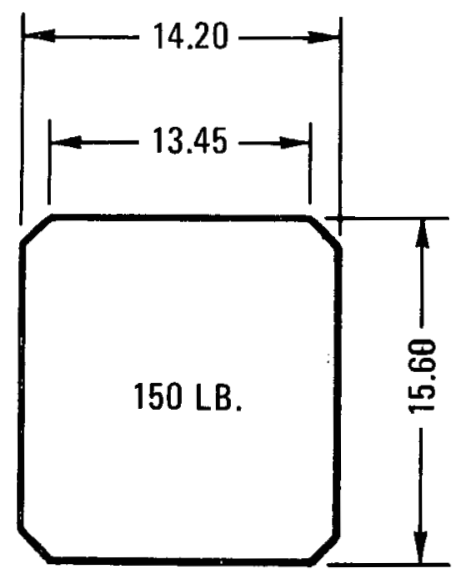

B) HALF HEIGHT BLOCK

F1g. 7. Block properties 
TABLE 2

SAMPLE PROBLEM DATA

\begin{tabular}{l|c|c}
\hline \multicolumn{1}{c|}{ Spring } & $\begin{array}{c}\text { Stiffness } \\
\text { (1b/in.) }\end{array}$ & $\begin{array}{c}\text { Damping } \\
(1 \mathrm{~b}-\text { sec/1n.) }\end{array}$ \\
\hline Between full and half height blocks & 13,333 & 35.3 \\
Between full height blocks & 10,000 & 37.4 \\
Between full height blocks and PCRV & 20,000 & 74.8 \\
Between half height blocks and PCRV & 10,000 & 37.4 \\
Support posts (two) & 4,000 & 7.8 \\
Dowels & 800 & 0.18 \\
\hline
\end{tabular}

Gaps

Block $1=0.05 \mathrm{in}$.

Blocks 2-5 $=0.50 \mathrm{in}$.

Dowels $=0.05 \mathrm{in}$.

Core support post spacing $=12.0 \mathrm{in}$.

Coefficient of friction $=0.2$

Friction force damping coefficient $=100 \mathrm{lb}-\mathrm{sec} / \mathrm{In}$.

Integration time step $=0.001 \mathrm{sec}$

Sinusoidal excitation

Horizontal $-0.5 \mathrm{~g}$ at $2.0 \mathrm{~Hz}$

Vertical $-0.25 \mathrm{~g}$ at $1.0 \mathrm{~Hz}$ 


\section{REFERENCES}

1. Lee, T. H., "Nonlinear Dynamic Analysis of a Stacked Fuel Column Subjected to Boundary Motion," GA-A12933, General Atomic Company, 29 August 1974.

2. Lee, T. H. and D. A. Wesley, "Nonlinear Seismic Response of a Series of Interacting Fuel Columns Consisting of Stacked Elements," GA-A13224, General Atomic Company, 1 June 1975.

3. Rickard, N. D., "Numerical Integration of Discontinuous Forcing Functions," unpublished data, General Atomic Company, 22 June 1976.

4. Belytschko, T., "Comparative Evaluation of Numerical Methods for Dynamic Structural Analysis," Methods for Dynamic Structural Analysis, Berlin, Paper No. T1/1, September 1975.

5. Gear, William, Numerical Initial Value Problems in Ordinary Differential Equations, 1971.

6. Thompson, R. W., "Revised COCO Mode1," unpublished data, General Atomic Company, 13 September 1976. 


\section{APPENDIX A}

INPUT DATA FORMS

A-1 


\begin{tabular}{|l|l|l|}
\hline WORD & & \\
\hline COLUMN & $1-72$ & \\
\hline FORMAT & A & \\
\hline & & \\
CARD & & \\
1 & Title & \\
\hline SYMBOL & Title & \\
\hline
\end{tabular}

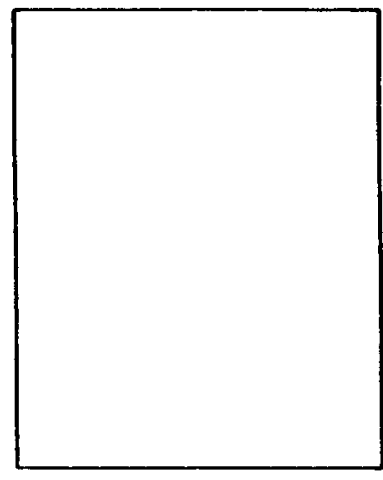

\begin{tabular}{|c|c|c|c|c|c|c|c|}
\hline WORD & 1 & 2 & 3 & 4 & 5 & \multicolumn{2}{|l|}{6} \\
\hline COLUMN & $5-6$ & $7-12$ & 18 & 24 & 30 & \multicolumn{2}{|l|}{36} \\
\hline FORMAT & I & I & $I$ & I & $I$ & \multicolumn{2}{|l|}{ I } \\
\hline $\begin{array}{c}\text { CARD } \\
2\end{array}$ & $\begin{array}{l}\text { No. of blocks } \\
1 \leq \mathrm{NE} \leq 14\end{array}$ & $\begin{array}{l}\text { Print } \\
\text { interval } \\
1 \leq \text { NPRT }\end{array}$ & $\begin{array}{l}\text { Not } \\
\text { used }\end{array}$ & \begin{tabular}{|l|} 
Punch \\
restart \\
deck \\
$0-$ yes \\
$1-$ no
\end{tabular} & $\begin{array}{l}\text { Integration } \\
\text { method } \\
0 \text { - central } \\
\text { difference } \\
1-2 \text { nd } \\
\text { order } \\
\text { Runge-Kutta }\end{array}$ & $\begin{aligned} & 0 \text { - No PCRV motion } \\
& 1 \text { - Sine dwell } \\
& 2 \text { - Horiz. accel. time } \\
& \text { history } \\
& 3 \text { - Vert. accel. time } \\
& \text { history } \\
& 4 \text { - Horiz \& Vert. accel }\end{aligned}$ & $\mid \begin{aligned} & \text { time history } \\
& 5 .- \text { Horiz displ. } \\
& \text { time history } \\
& 6 \text { - } \\
& \text { Vert. displ. } \\
& \text { time history } \\
& 7 \text { - } \begin{array}{l}\text { Hor. dver. dis } \\
\text { time histary }\end{array} \\
&\end{aligned}$ \\
\hline SYMBOL & $\mathrm{NE}$ & NPRT & & NSTRT & IMETH & \multicolumn{2}{|l|}{ IGM } \\
\hline
\end{tabular}

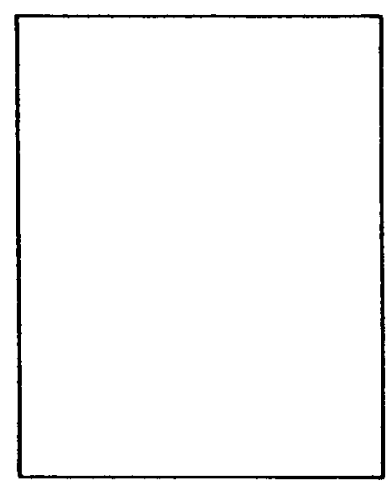

\begin{tabular}{|l|l|l|l|l|}
\hline WORD & 7 & 8 & 9 & \\
\hline COLUMN & 42 & 48 & $53-54$ & \\
\hline FORMAT & 2 & I & I & \\
\hline & Initial & Initial & No of & \\
& angular & velocities & $\begin{array}{l}\text { locked } \\
\text { degrees }\end{array}$ & \\
displacements & $0-$ no & \\
CARD & $0-$ no & $1-$ yes & of foedom & \\
& $1-$ yes & & & \\
\hline SYMBOL & IQD & IQON & NLDOF & \\
\hline
\end{tabular}

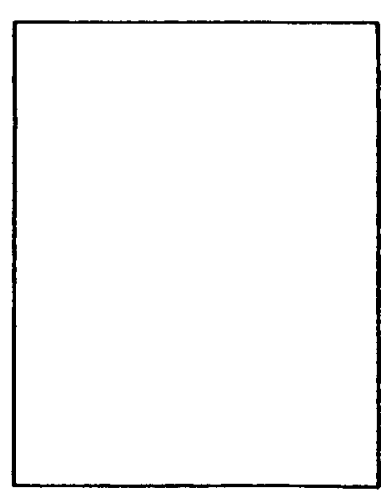




\begin{tabular}{|c|c|c|c|c|c|}
\hline WORD & 1 & 2 & 3 & 4 & $5-12$ \\
\hline COLUMN & $1-6$ & $7-12$ & $13-18$ & $19-24$ & $25-72$ \\
\hline FORMAT & I6 & I6 & I6 & I6 & 816 \\
\hline $\begin{array}{c}\text { CARD } \\
3 \\
\text { Use only if }\end{array}$ & $\begin{array}{l}\text { Block No. } \\
\text { of first } \\
\text { locked degree } \\
\text { of freedom }\end{array}$ & $\begin{array}{l}\text { Component of } \\
\text { first locked } \\
\text { degree of } \\
\text { freedom }\end{array}$ & $\begin{array}{l}\text { Block No. } \\
\text { of second } \\
\text { locked degree } \\
\text { of freedom }\end{array}$ & $\begin{array}{l}\text { Component of second } \\
\text { locked degree of } \\
\text { freedom }\end{array}$ & Addition pairs of data \\
\hline NLDOF $>0$ & & & & & $-\operatorname{LBLK}(3), \operatorname{LCOMP}(3) \ldots$ LBLK \\
\hline SYMBOL & $\operatorname{LBLK}(1)$ & $\operatorname{LCOMP}(1)$ & LBLK (2) & LCOMP (2) & (NLDOF), LCOMP (NLDOF) \\
\hline
\end{tabular}

Enter NLDOF pairs

of block and component numbers.

Enter up to 6

pairs per card.

Use as many cards

as required.

\begin{tabular}{|l|l|}
\hline WORD & \\
\hline COLUMN & \\
\hline FORMAT & \\
\hline & \\
\hline \multirow{2}{*}{ CARD } & \\
\hline & \\
\hline SYMBOL & \\
\hline
\end{tabular}

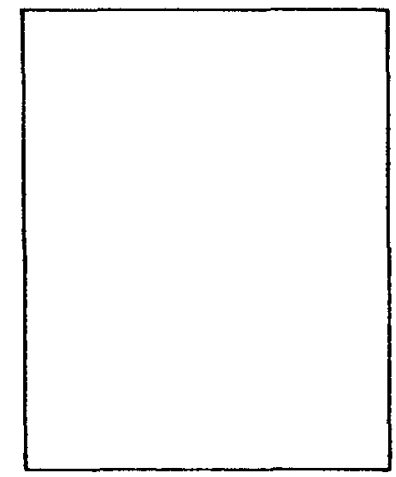

\begin{tabular}{|l|l|}
\hline WORD & \\
\hline COLUMN & \\
\hline FORMAT & \\
\hline & \\
CARD & \\
& \\
\hline SYMBOL & \\
\hline
\end{tabular}

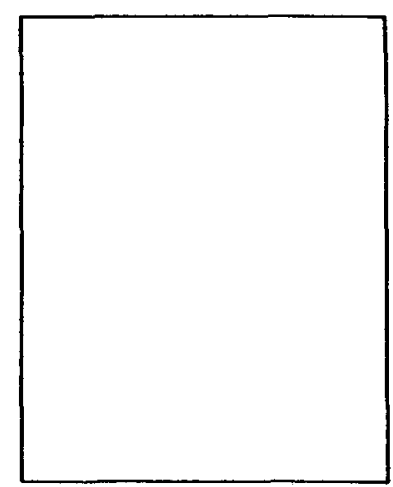




\begin{tabular}{|l|l|l|l|l|}
\hline WORD & 1 & 2 & 3 & \\
\hline COLUMN & $1-12$ & $13-24$ & $25-36$ & \\
\hline FORMAT & F & F & F & \\
\hline & $\begin{array}{l}\text { Starting } \\
\text { time } \\
\text { (sec.) }\end{array}$ & $\begin{array}{l}\text { Ending } \\
\text { time } \\
\text { (sec.) }\end{array}$ & $\begin{array}{l}\text { Integration } \\
\text { time step } \\
(\text { sec. })\end{array}$ & \\
4 & & TEND & HH & \\
\hline SYMBOL & TO & & \\
\hline
\end{tabular}

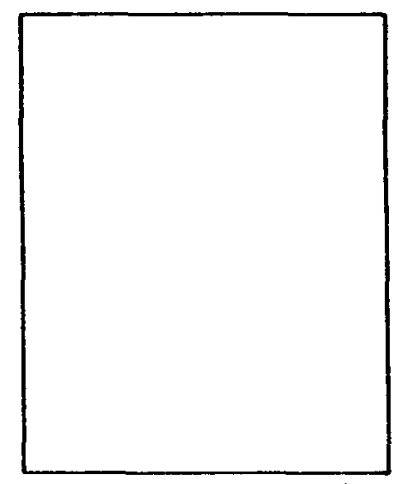

\begin{tabular}{|c|c|c|c|c|c|c|c|}
\hline WORD & 1 & 2 & 3 & 4 & 5 & 6 & \\
\hline COLUMN & $1-12$ & $13-24$ & $25-36$ & $37-48$ & $49-60$ & $61-72$ & \\
\hline FORMAT & $\mathbf{F}$ & $\mathbf{F}$ & $\mathbf{F}$ & $\mathrm{F}$ & F & $F$ & \\
\hline & $\begin{array}{l}\text { Element } \\
\text { geometric }\end{array}$ & $\begin{array}{l}\text { Rotational } \\
\text { spring stiff- }\end{array}$ & \multicolumn{4}{|c|}{ Initial conditions for PCRV } & \\
\hline $\begin{array}{c}\text { CARD } \\
5\end{array}$ & $\begin{array}{l}\text { width } \\
\text { (in.) }\end{array}$ & $\begin{array}{l}\text { ness for } \\
\text { bottom block } \\
(\mathrm{lb} / \mathrm{rad})\end{array}$ & $\begin{array}{l}\text { Horizontal } \\
\text { displacement } \\
\text { (in.) }\end{array}$ & $\begin{array}{l}\text { Horizontal } \\
\text { velocity } \\
(1 n . / \mathrm{sec})\end{array}$ & $\begin{array}{l}\text { Vertical } \\
\text { displacement } \\
\text { (in.) }\end{array}$ & $\begin{array}{l}\text { Vertical } \\
\text { velocity } \\
(\text { in./sec) }\end{array}$ & \\
\hline SYMBOL & BG & RSPRING & UO & UOD & WO & WOD & \\
\hline
\end{tabular}

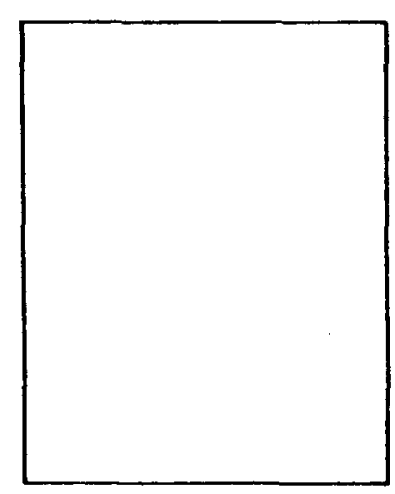

\begin{tabular}{|l|l|}
\hline WORD & \\
\hline COLUMN & \\
\hline FORMAT & \\
\hline & \\
CARD & \\
& \\
\hline SYMBOL & \\
\hline
\end{tabular}

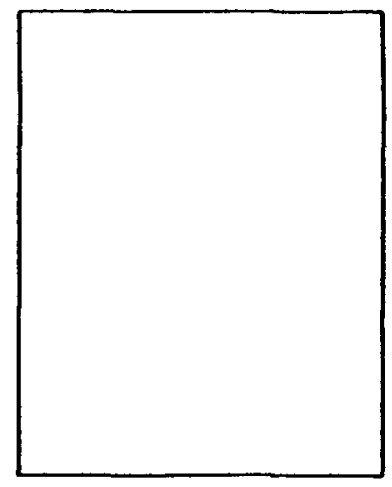

D. 


\begin{tabular}{|c|c|c|c|c|c|c|c|}
\hline WORD & 1 & 2 & 3 & 4 & 5 & 6 & 7 \\
\hline COLUMN & $1-10$ & $11-20$ & $21-30$ & $31-40$ & $41-50$ & $51-60$ & $61-70$ \\
\hline FORMAT & $\mathrm{F}$ & $\bar{F}$ & $\mathrm{~F}$ & $F$ & $F$ & $F$ & $\mathrm{~F}$ \\
\hline $\begin{array}{c}\text { CARD } \\
6\end{array}$ & $\begin{array}{l}\text { Vertical } \\
\text { corner } \\
\text { spring } \\
\text { stiffness } \\
\text { (1b/in.) }\end{array}$ & $\begin{array}{l}\text { Vertical } \\
\text { corner } \\
\text { spring } \\
\text { damping } \\
(1 b-\sec / \text { in.) }\end{array}$ & $\begin{array}{l}\text { Vertical } \\
\text { center } \\
\text { spring } \\
\text { stiffness } \\
(1 b / i n .)\end{array}$ & $\begin{array}{l}\text { Vertical } \\
\text { center } \\
\text { spring } \\
\text { damping } \\
(1 b-\text { sec/in.) }\end{array}$ & $\begin{array}{l}\text { Horizontal } \\
\text { corner } \\
\text { spring } \\
\text { stiffness } \\
(1 b / i n .)\end{array}$ & $\begin{array}{l}\text { Horizontal } \\
\text { corner } \\
\text { spring } \\
\text { damping } \\
(1 b-\sec / \text { in.) }\end{array}$ & $\begin{array}{l}\text { Horizontal } \\
\text { center } \\
\text { spring } \\
\text { stiffness } \\
\text { (1b/in.) }\end{array}$ \\
\hline SYMBOL & VCRNS (I) & $\operatorname{VCRND}(I)$ & $\operatorname{VCENS~(I)~}$ & $\operatorname{VCEND}(\mathrm{I})$ & $\operatorname{HCRNS}(\mathrm{I})$ & HCRND (I) & $\operatorname{HCENS}(\mathrm{I})$ \\
\hline
\end{tabular}

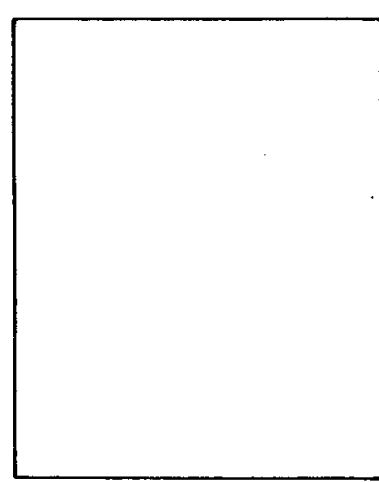

\begin{tabular}{|l|l|l|}
\hline WORD & 8 & \\
\hline COLUMN & $71-80$ & \\
\hline FORMAT & F & \\
\hline & Horizontal & \\
& center & \\
& spring & \\
CARD & damping & \\
6 (Cont.) & (lb-sec/in.) & \\
\hline SYMBOL & HCEND(I) & \\
\hline
\end{tabular}

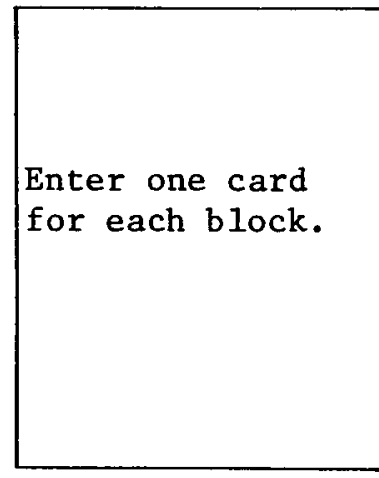

\begin{tabular}{|l|l|l|l|l|l|l|l|}
\hline WORD & 1 & 2 & 3 & 4 & 5 & 6 & $7-12$ \\
\hline COLUMN & $1-6$ & $7-12$ & $13-18$ & $19-24$ & $25-30$ & $31-36$ & $37-42, \ldots, 67-72$ \\
\hline FORMAT & F & F & F & F & F & F & \\
\hline $\begin{array}{l}\text { CARD } \\
7\end{array}$ & $\begin{array}{l}\text { Upper left } \\
\text { corner } \\
\text { horizontal } \\
\text { gap } \\
\text { Block 1 } \\
\text { (in.) }\end{array}$ & Block 2 & Block 3 & Block 4 & Block 5 & Block 6 & Blocks 7-12 \\
\hline SYMBOL & GAP(3,1) & $\operatorname{GAP}(3,2)$ & $\operatorname{GAP}(3,3)$ & $\operatorname{GAP}(3,4)$ & $\operatorname{GAP}(3,5)$ & $\operatorname{GAP}(3,6)$ & $\operatorname{GAP}(3,7)-\operatorname{GAP}(3,12)$ \\
\hline
\end{tabular}

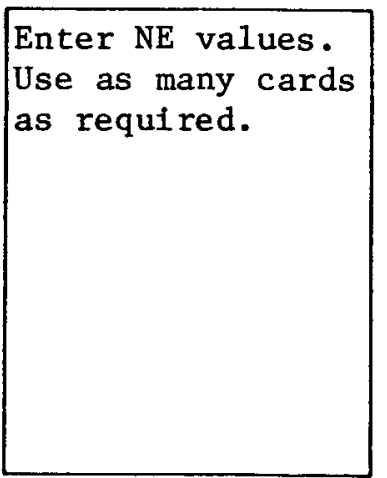




\begin{tabular}{|l|l|l|l|l|l|l|l|}
\hline WORD & 1 & 2 & 3 & 4 & 5 & 6 & $7-12$ \\
\hline COLUMN & $1-6$ & $7-12$ & $13-18$ & $19-24$ & $25-30$ & $31-36$ & $37-42, \ldots, 67-72$ \\
\hline FORMAT & F & F & F & F & F & F & \\
\hline $\begin{array}{c}\text { CARD } \\
8\end{array}$ & $\begin{array}{l}\text { Lower left } \\
\text { corner } \\
\text { horizontal } \\
\text { gap } \\
\text { Block 1 } \\
\text { in.) }\end{array}$ & Block 2 & Block 3 & Block 4 & Block 5 & Block 6 & Blocks $7-12$ \\
\hline SYMBOL & $\operatorname{GAP}(4,1)$ & $\operatorname{GAP}(4,2)$ & $\operatorname{GAP}(4,3)$ & $\operatorname{GAP}(4,4)$ & $\operatorname{GAP}(4,5)$ & $\operatorname{GAP}(4,6)$ & $\operatorname{GAP}(4,7)-\operatorname{GAP}(4,12)$ \\
\hline
\end{tabular}

Enter NE values. Use as many cards as required

\begin{tabular}{|l|l|}
\hline WORD & \\
\hline COLUMN & \\
\hline FORMAT & \\
\hline & \\
\hline & \\
CaRD & \\
& \\
\hline SYMBOL & \\
\hline
\end{tabular}

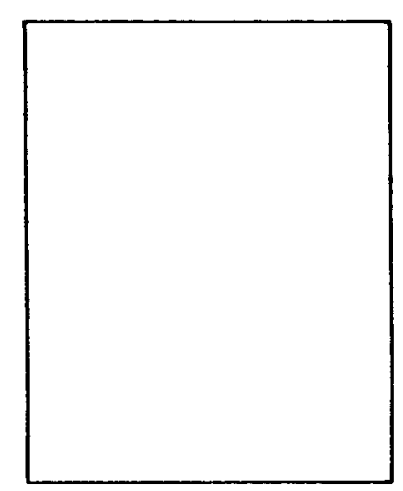

\begin{tabular}{|l|l|}
\hline WORD & \\
\hline COLUMN & \\
\hline FORMAT & \\
\hline & \\
CARD & \\
& \\
\hline SYMBOL & \\
\hline
\end{tabular}

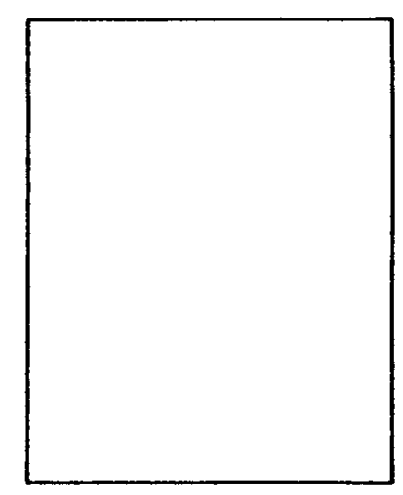

O 


\begin{tabular}{|l|l|l|l|l|l|l|l|}
\hline WORD & 1 & 2 & 3 & 4 & 5 & 6 & $7-12$ \\
\hline C.OLUMN & $1-6$ & $7-12$ & $13-18$ & $19-24$ & $25-30$ & $31-36$ & $37-42,---, 67-72$ \\
\hline FORMAT & F & F & F & F & F & F & F \\
\hline $\begin{array}{c}\text { CARD } \\
9\end{array}$ & $\begin{array}{l}\text { Upper right } \\
\text { corner hori- } \\
\text { zonta1 gap } \\
\text { Block 1 } \\
\text { (in.) }\end{array}$ & Block 2 & Block 3 & Block 4 & Block 5 & Block 6 & Blocks 7-12 \\
\hline SYMBOL & $\operatorname{GAP}(1,1)$ & $\operatorname{GAP}(1,2)$ & $\operatorname{GAP}(1,3)$ & $\operatorname{GAP}(1,4)$ & $\operatorname{GAP}(1,5)$ & $\operatorname{GAP}(1,6)$ & $\operatorname{GAP}(1,7)-\operatorname{GAP}(1,12)$ \\
\hline
\end{tabular}

Enter NE values. Use as many cards as required.

Enter NE values. Use as many cards as required.

\begin{tabular}{|l|l|l|l|l|l|l|l|}
\hline WORD & 1 & 2 & 3 & 4 & 5 & 6 & $7-12$ \\
\hline COLUMN & $1-6$ & $7-12$ & $13-18$ & $19-24$ & $25-30$ & $31-36$ & $37-42, \ldots, 67-72$ \\
\hline FORMAT & F & F & F & F & F & F & F \\
\hline $\begin{array}{l}\text { CARD } \\
10\end{array}$ & $\begin{array}{l}\text { Lower right } \\
\text { corner hori- } \\
\text { zontal gap } \\
\text { Block 1 } \\
\text { (in.) }\end{array}$ & Block 2 & Block 3 & Block 4 & Block 5 & Block 6 & Blocks 7-12 \\
\hline SYMBOL & GAP(2,1) & GAP(2,2) & GAP(2,3) & GAP(2,4) & GAP(2,5) & GAP(2,6) & GAP (2,7) -GAP(2,12) \\
\hline
\end{tabular}

\begin{tabular}{|l|l|l|l|l|l|l|}
\hline WORD & 1 & 2 & 3 & 4 & 5 & 6 \\
\hline COLUMN & $1-12$ & $13-24$ & $25-36$ & $26-48$ & $49-60$ & $61-72$ \\
\hline FORMAT & F & F & F & F & F & F \\
\hline & $\begin{array}{l}\text { Dowe1 } \\
\text { stiffness } \\
\text { (1b/in.) }\end{array}$ & $\begin{array}{l}\text { Dowe1 } \\
\text { damping } \\
\text { coefficient } \\
(11\end{array}$ & $\begin{array}{l}\text { Right } \\
\text { side } \\
\text { dowe1 gap } \\
(\text { in. })\end{array}$ & $\begin{array}{l}\text { Left } \\
\text { side } \\
\text { dowe1 gap } \\
(\text { in. })\end{array}$ & $\begin{array}{l}\text { Coefficient } \\
\text { of friction }\end{array}$ & $\begin{array}{l}\text { Friction } \\
\text { force } \\
\text { damping } \\
\text { coefficient } \\
(1 b-\sec / \text { in. })\end{array}$ \\
\hline SYMBOL & SK & CDW & GAP1 & GAP2 & COF & SLP \\
\hline
\end{tabular}

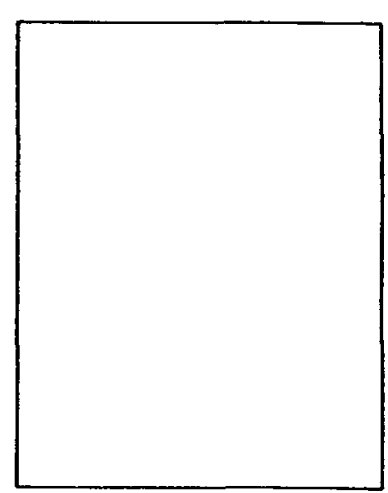




\begin{tabular}{|l|l|l|l|l|l|}
\hline WORD & 1 & 2 & 3 & 4 & \\
\hline COLUMN & $1-12$ & $13-24$ & $25-36$ & $37-48$ & \\
\hline FORMAT & F & F & F & F & \\
\hline & $\begin{array}{l}\text { Horizontal } \\
\text { g level } \\
(\mathrm{g})\end{array}$ & $\begin{array}{l}\text { Horizontal } \\
\text { frequency } \\
(\mathrm{Hz})\end{array}$ & $\begin{array}{l}\text { Vertical } \\
\text { g level } \\
(\mathrm{g})\end{array}$ & $\begin{array}{l}\text { Vertical } \\
\text { frequency } \\
\text { (He) }\end{array}$ & \\
$\begin{array}{c}\text { CARD } \\
12\end{array}$ & & & & \\
Use only if & & FREQU & AMPLW & FREQW & \\
IGM 1 & & AMPLU & & \\
\hline SYMBOL & & &
\end{tabular}

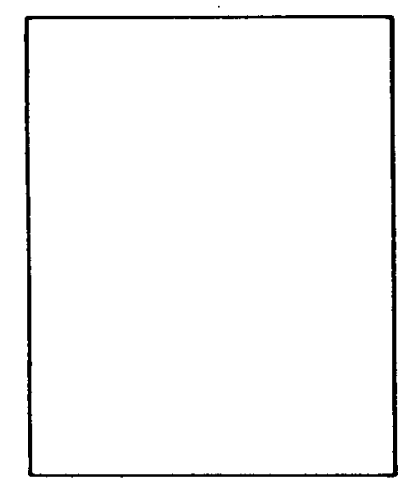

\begin{tabular}{|l|l|l|l|l|l|l|l|}
\hline WORD & 1 & 2 & 3 & 4 & 5 & 6 & \\
\hline COLUMN & $1-12$ & $13-24$ & $25-36$ & $37-48$ & $49-60$ & $61-72$ & \\
\hline FORMAT & F & F & F & F & F & F & \\
\hline $\begin{array}{c}\text { CARD } \\
13\end{array}$ & $\begin{array}{l}\text { Height } \\
\text { of } \\
\text { block 1 } \\
\text { (in.) }\end{array}$ & Block 2 & Block 3 & Block 4 & Block 5 & Block 6 & \\
\hline SYMBOL & H(1) & H(2) & H(3) & H(4) & H(5) & H(6) & \\
\hline
\end{tabular}

Enter NE values. Use as many cards as required.

Enter NE values. Use as many cards

as required.

\begin{tabular}{|l|l|l|l|l|l|l|l|}
\hline WORD & 1 & 2 & 3 & 4 & 5 & 6 & \\
\hline COLUMN & $1-12$ & $13-24$ & $25-36$ & $37-48$ & $49-60$ & $61-72$ & \\
\hline FORMAT & & & & & & & \\
\hline $\begin{array}{c}\text { CARD } \\
14\end{array}$ & $\begin{array}{l}\text { Rocking } \\
\text { width of } \\
\text { block 1 } \\
\text { (in.) }\end{array}$ & Block 2 & Block 3 & Block 4 & Block 5 & Block 6 & \\
\hline SYMBOL & $\mathrm{B}(1)$ & $\mathrm{B}(2)$ & $\mathrm{B}(3)$ & $\mathrm{B}(4)$ & $\mathrm{B}(5)$ & $\mathrm{B}(6)$ & \\
\hline
\end{tabular}




\begin{tabular}{|l|l|l|l|l|l|l|l|}
\hline WORD & 1 & 2 & 3 & 4 & 5 & 6 & \\
\hline COLUMN & $1-12$ & $13-24$ & $25-36$ & $37-48$ & $49-60$ & $61-72$ & \\
\hline FORMAT & F & F & F & F & F & F & \\
\hline $\begin{array}{l}\text { CARO } \\
15\end{array}$ & $\begin{array}{l}\text { Wlock of 1 } \\
\text { (1b) }\end{array}$ & Block 2 & Block 3 & Block 4 & Block 5 & Block 6 & \\
\hline SYMBOL & WT(1) & WT(2) & WT(3) & WT(4) & WT (5) & WT (6) & \\
\hline
\end{tabular}

Enter NE value.

Use as many cards as required.

Enter NE values. Use as many cards as required.

Enter NE values. Use as many cards as required.

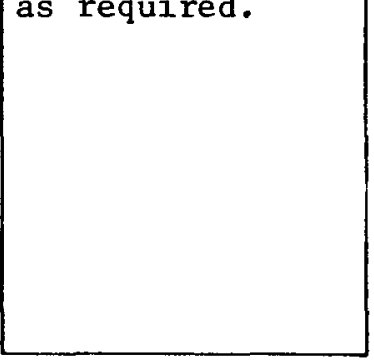

CARD

17 Use only if IQDN $=1$ SYMBOL

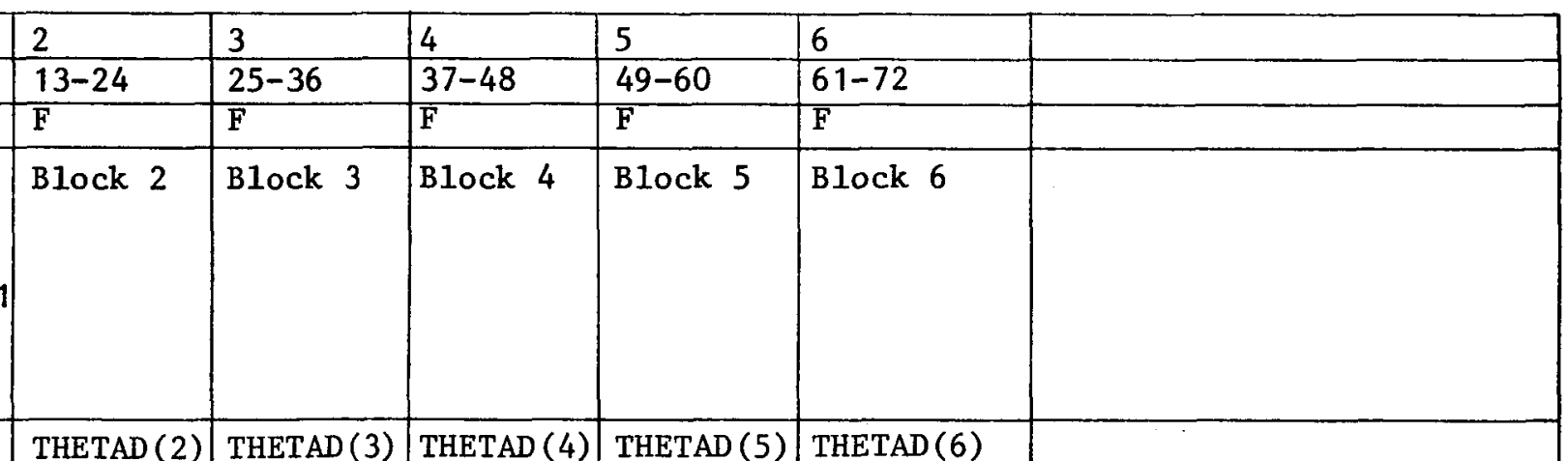

\begin{tabular}{|c|c|}
\hline WORD & 1 \\
\hline COLUMN & 1 \\
\hline FORMAT & F \\
& In \\
& an \\
CARD & vel \\
17 & of \\
Use onIy if & (ra \\
IQDN = 1 & \\
\hline SYMBOL & TH \\
\hline
\end{tabular}

\begin{tabular}{l|l}
\hline 1 & 2 \\
\hline $1-12$ & 13 \\
\hline F & F \\
\hline $\begin{array}{l}\text { Initial } \\
\text { angular } \\
\text { velocity } \\
\text { of block } \\
\text { (rad/sec) }\end{array}$ & B \\
\hline THETAD (1) & T
\end{tabular}
displacement of block 1 Use only if $\mathrm{QD}=1$

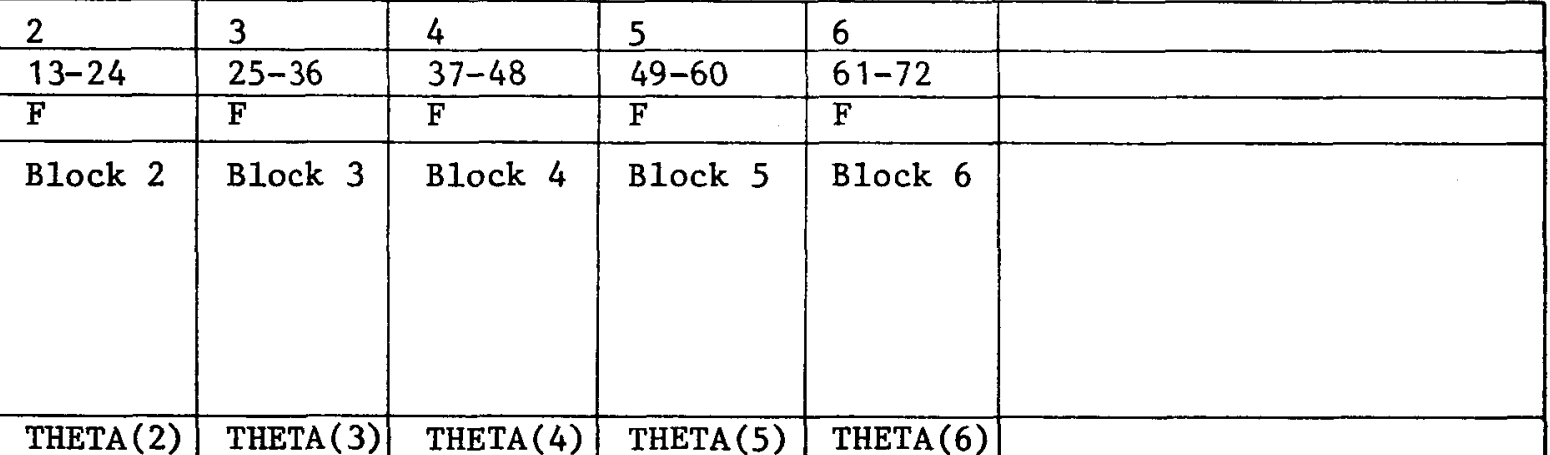

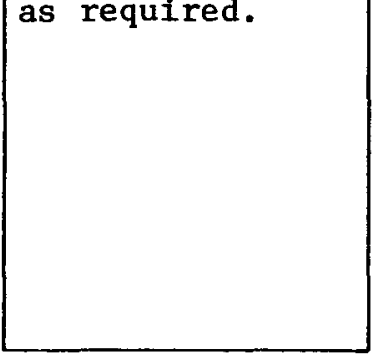




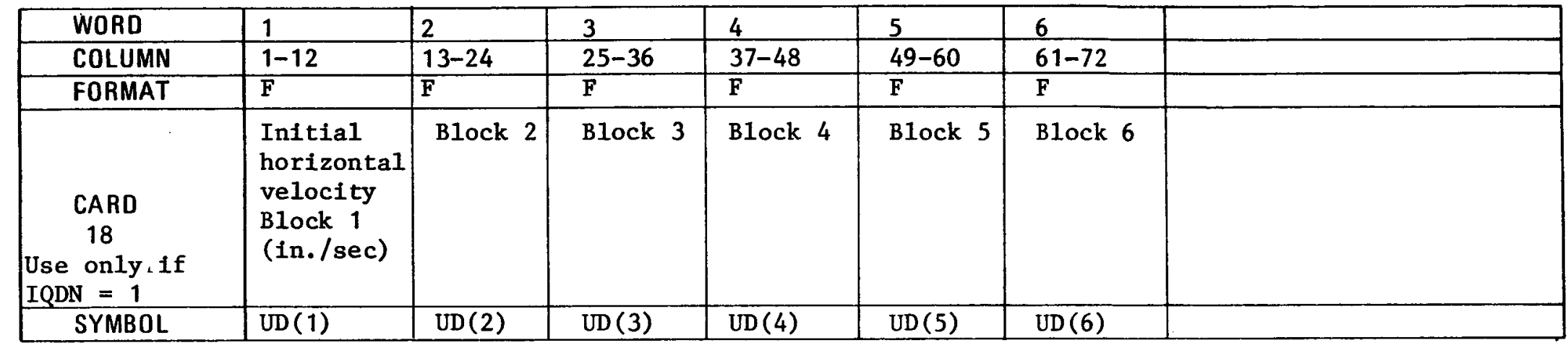

Enter NE values. Use as many cards as required.

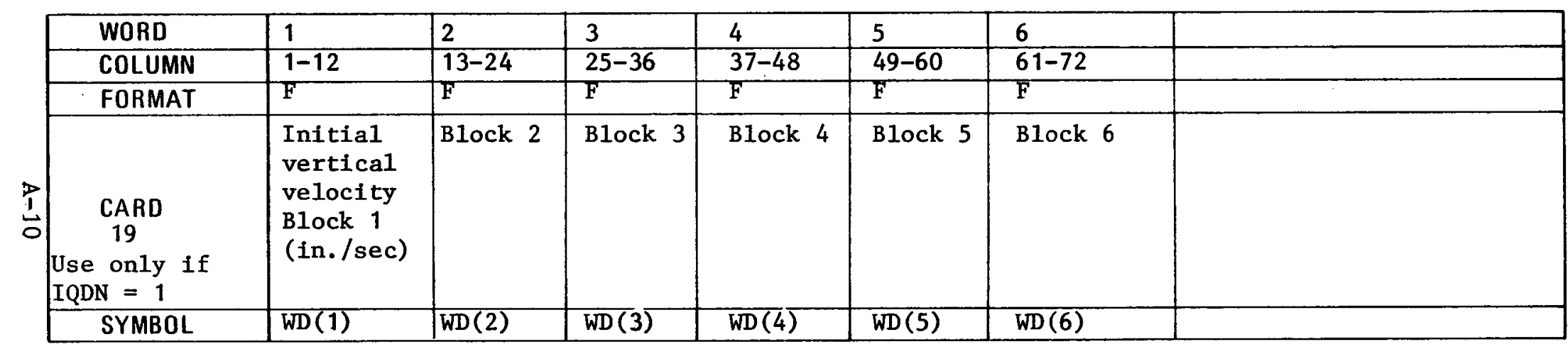

Enter NE values. Use as many cards as required.

\begin{tabular}{|c|l|l|l|l|l|}
\hline WORD & 1 & 2 & 3 & 4 & \\
\hline COLUMN & $1-12$ & $13-24$ & $25-36$ & $37-48$ & \\
\hline FORMAT & I & F & F & F & \\
\hline & $\begin{array}{l}\text { No. of } \\
\text { horizontal } \\
\text { displacement } \\
\text { time history } \\
\text { points }\end{array}$ & $\begin{array}{l}\text { Scaling } \\
\text { factor }\end{array}$ & G level & $\begin{array}{l}\text { Time step } \\
\text { between } \\
\text { points } \\
\text { only if }\end{array}$ \\
$\begin{array}{l}\text { Usec } \\
\text { IGM or } 7\end{array}$ & NINPU & FACTOR & SCALE & DELT & \\
\hline SYMBOL & & & & \\
\hline
\end{tabular}

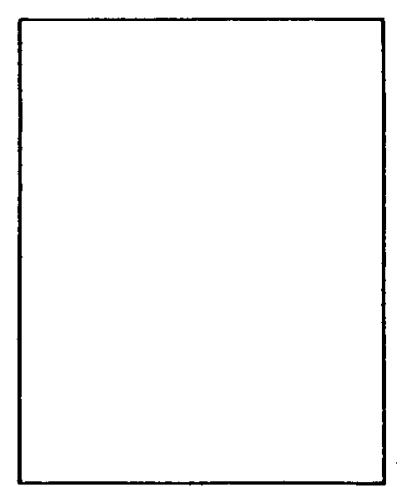




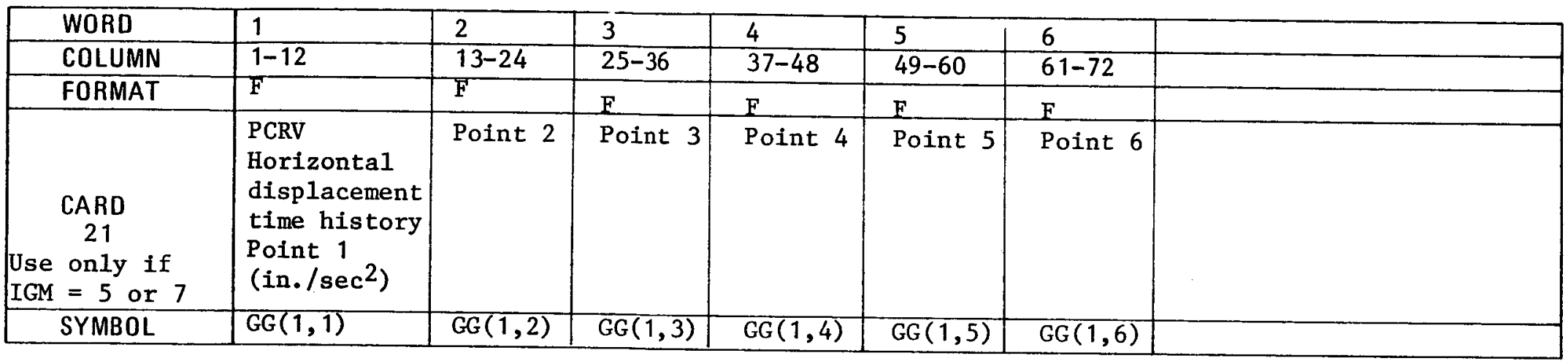

\begin{tabular}{|l|}
\hline Enter NINPU \\
values. Use as \\
many cards as \\
required. \\
\end{tabular}

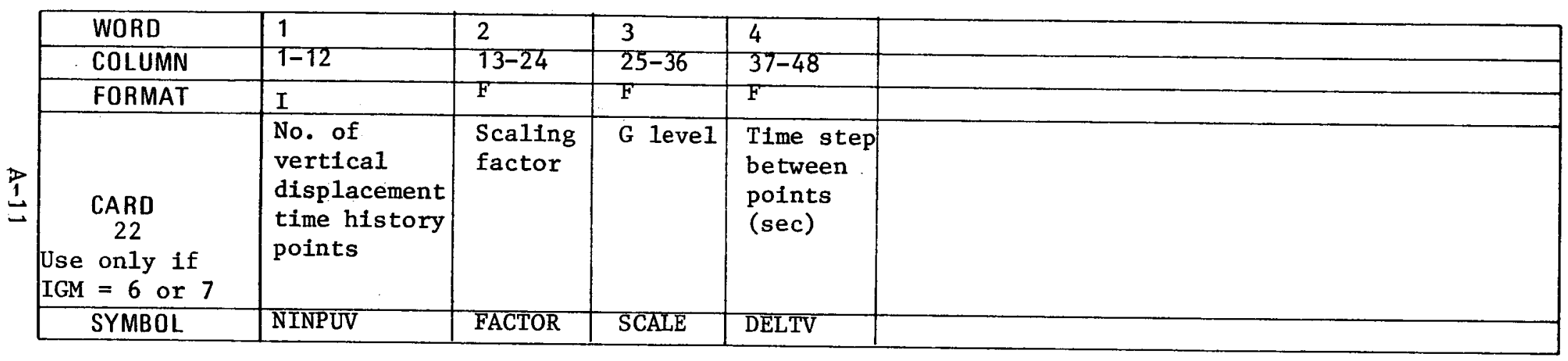

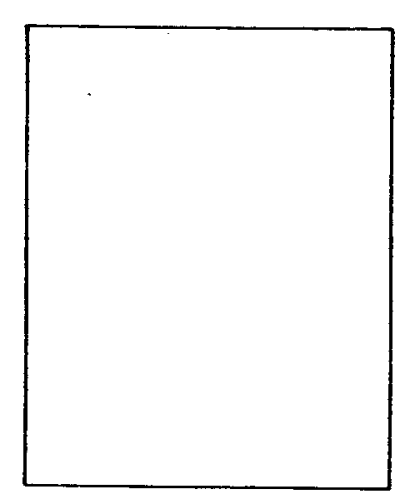

\begin{tabular}{|c|c|c|c|c|c|c|c|}
\hline WORD & 1 & 2 & 3 & 4 & 5 & 6 & \\
\hline COLUMN & $1-12$ & $13-24$ & $25-36$ & $37-48$ & $49-60$ & $61-72$ & \\
\hline FORMAT & $F$ & $F$ & $F^{\prime}$ & $F$ & $F$ & $F$ & \\
\hline $\begin{array}{c}\text { CARD } \\
23 \\
\text { Use only if } \\
\text { IGM }=6 \text { or } 7\end{array}$ & $\begin{array}{l}\text { PCRV } \\
\text { vertical } \\
\text { displacement } \\
\text { time history } \\
\text { Point } 1 \\
\left(\text { in. } / \mathrm{sec}^{2}\right)\end{array}$ & Point 2 & Point 3 & Point 4 & Point 5 & Point 6 & \\
\hline SYMBOL & $\operatorname{GG}(4,1)$ & $\operatorname{GG}(4,2)$ & $\mathrm{GG}(4,3)$ & $\mathrm{GG}(4,4)$ & $G G(4,5)$ & $\operatorname{GG}(4,6)$ & \\
\hline
\end{tabular}

Enter NINPUV
values. Use as
many cards as
required.




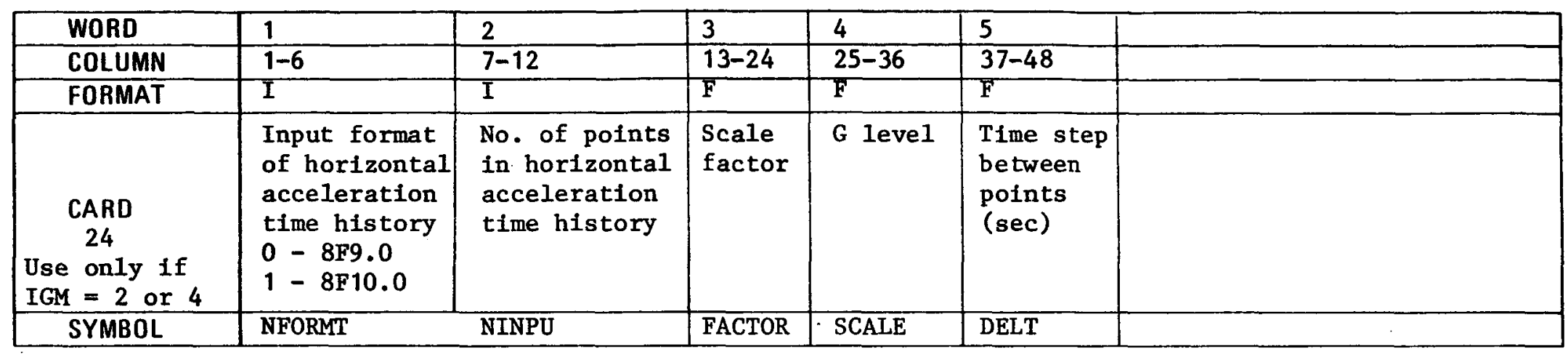

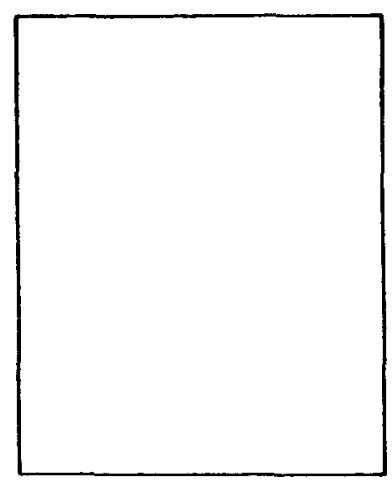

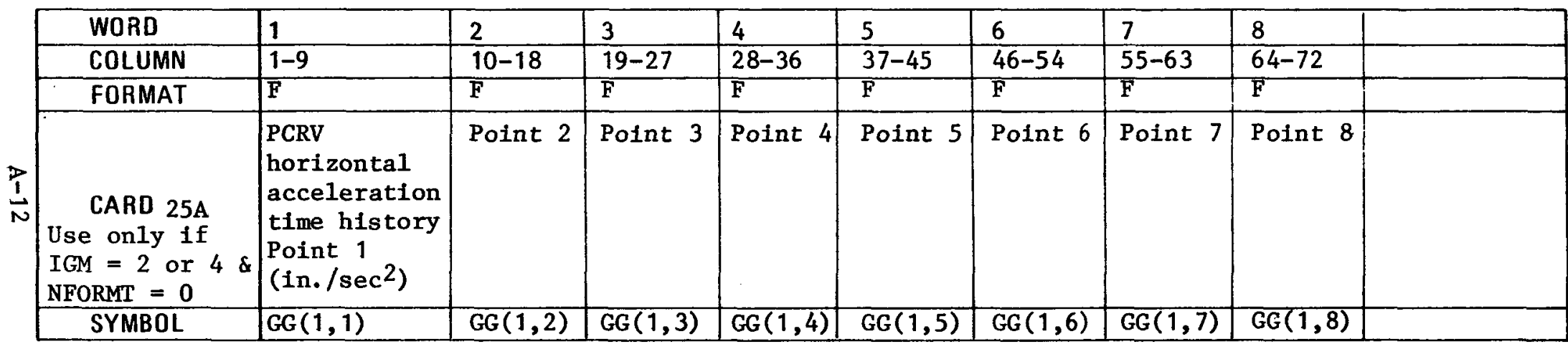

See note on card 21.

\begin{tabular}{|c|c|c|c|c|c|c|c|c|c|}
\hline WORD & 1 & 2 & 3 & 4 & 5 & 6 & 7 & 8 & \\
\hline COLUMN & $\sqrt{1-10}$ & $11-20$ & $21-30$ & $31-40$ & $41-50$ & $51-60$ & $61-70$ & $71-80$ & \\
\hline FORMAT & $\bar{F}$ & $\overline{\mathrm{F}}$ & F & F & $F$ & $F$ & $F$ & $F$ & \\
\hline $\begin{array}{l}\text { CARD 25B } \\
\text { Use only if } \\
\text { IGM }=2 \text { or } 4 \\
\text { NFORMT }=1\end{array}$ & $\begin{array}{l}\text { PCRV } \\
\text { horizontal } \\
\text { acceleration } \\
\text { time history } \\
\text { Point } 1 \\
\left(\text { in. } / \sec ^{2}\right)\end{array}$ & Point 2 & Point 3 & Point 4 & Point 5 & Point 6 & Point 7 & Point 8 & \\
\hline SYMBOL & $G G(1,1)$ & $G G(1,2)$ & $G G(1,3)$ & GG $(1,4)$ & GG $(1,5)$ & $G G(1,6)$ & $G G(1,7)$ & $\mathrm{GG}(1,8)$ & \\
\hline
\end{tabular}

See note on

card 21 . 


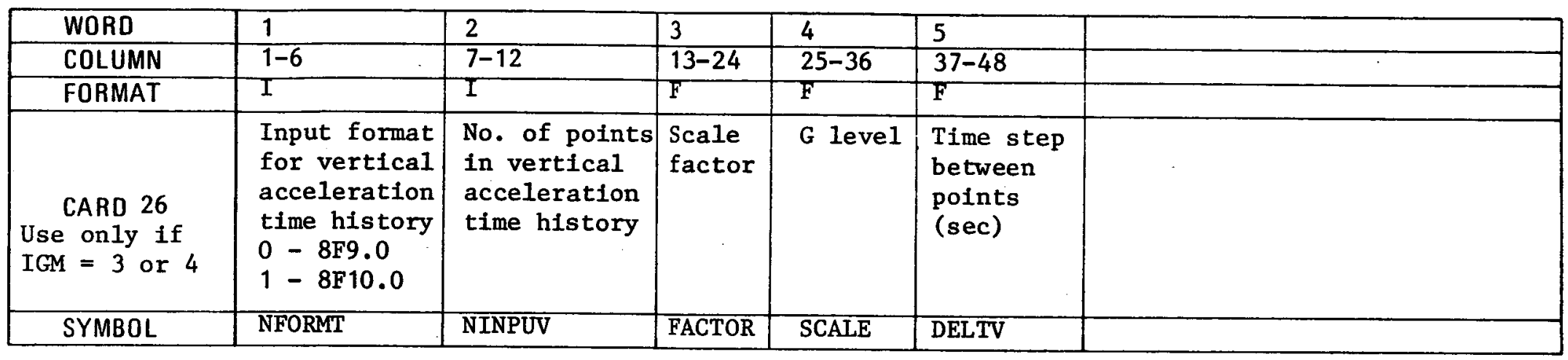

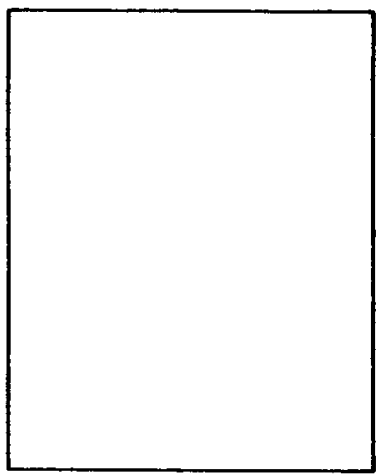

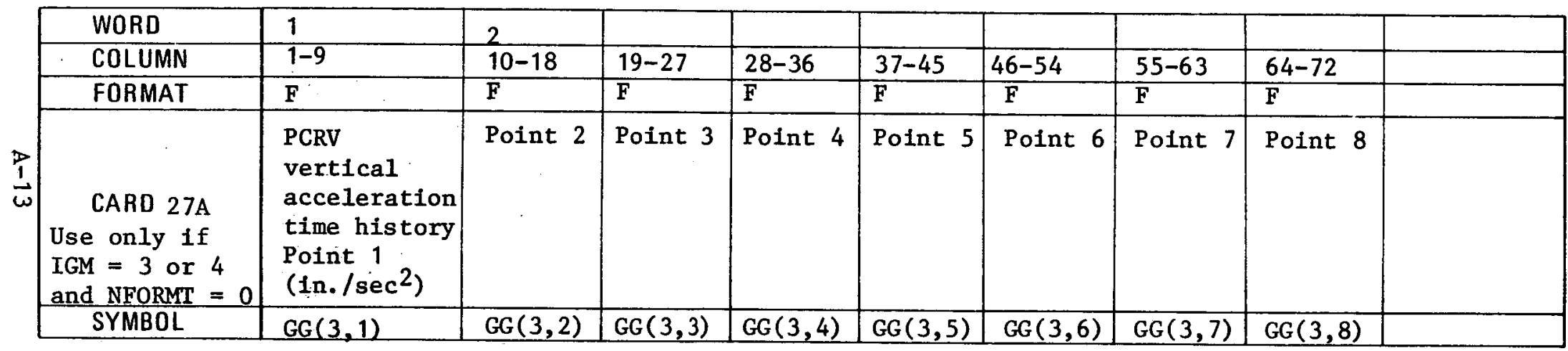

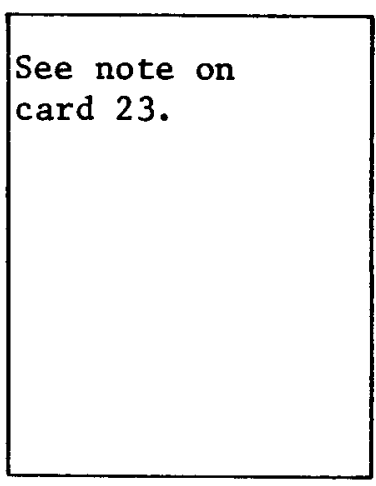

\begin{tabular}{|c|l|l|l|l|l|l|l|l|l|}
\hline WORD & 1 & 2 & 3 & 4 & 5 & 6 & 7 & 8 & \\
\hline COLUMN & $1-10$ & $11-20$ & $21-30$ & $31-40$ & $31-50$ & $51-60$ & $61-70$ & $71-80$ & \\
\hline FORMAT & F & F & F & F & F & F & F & F & \\
\hline $\begin{array}{l}\text { CARD 27B } \\
\text { on1y if } \\
\text { vertical } \\
\text { acceleration } \\
\text { time history } \\
\text { and NFORMT 4 1 }\end{array}$ & $\begin{array}{l}\text { Point 1 } \\
\text { (in./sec }\end{array}$ & Point 2 & Point 3 & Point 4 & Point 5 & Point 6 & Point 7 & Point 8 & \\
\hline SYMBOL & GG(3,1) & GG(3,2) & GG(3,3) & GG(3,4) & GG(3,5) & GG(3,6) & GG(3,7) & GG(3,8) & \\
\hline
\end{tabular}

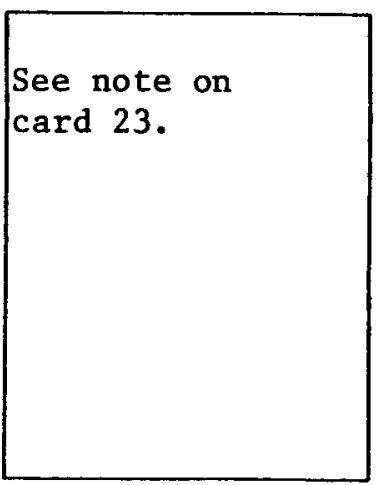




\begin{tabular}{|c|l|l|}
\hline WORD & 1 & \\
\hline COLUMN & $1-10$ & \\
\hline FORMAT & I & \\
\hline Restart Deck & $\begin{array}{l}\text { Integration } \\
\text { time step } \\
\text { counter }\end{array}$ & \\
$\begin{array}{c}\text { CARD } \\
28 \\
\text { (Optiona1) }\end{array}$ & & \\
\hline SYMBOL & LL & \\
\hline
\end{tabular}

The restart deck is automatically punch by the code if NSTRT $=0$.

\begin{tabular}{|c|c|c|c|c|c|}
\hline WORD & 1 & 2 & 3 & 4 & 5 \\
\hline COLUMN & $1-16$ & $17-32$ & $33-48$ & $49-64$ & $65-80$ \\
\hline FORMAT & $\mathbf{F}$ & $F$ & $\mathrm{~F}$ & $F$ & $F$ \\
\hline $\begin{array}{c}\text { Restart Deck } \\
\text { CARD } \\
29 \\
\text { (Optional) }\end{array}$ & $\begin{array}{l}\text { Angular } \\
\text { displacement } \\
\text { of block } 1 \\
\text { (rad) }\end{array}$ & $\begin{array}{l}\text { Angular } \\
\text { displacement } \\
\text { of block NE } \\
\text { (rad) }\end{array}$ & $\begin{array}{l}\text { Horizontal } \\
\text { displacement } \\
\text { of block } 1 \\
\text { (in.) }\end{array}$ & $\begin{array}{l}\text { Horizontal } \\
\text { displacement } \\
\text { of block NE } \\
\text { (in.) }\end{array}$ & $\begin{array}{l}\text { Vertical } \\
\text { displacement } \\
\text { of block } 1 \\
\text { (in.) }\end{array}$ \\
\hline SYMBOL & THETA (1) & THETA(NE) & $\mathrm{U}(1)$ & $\mathrm{U}(\mathrm{NE})$ & $W(1)$ \\
\hline
\end{tabular}

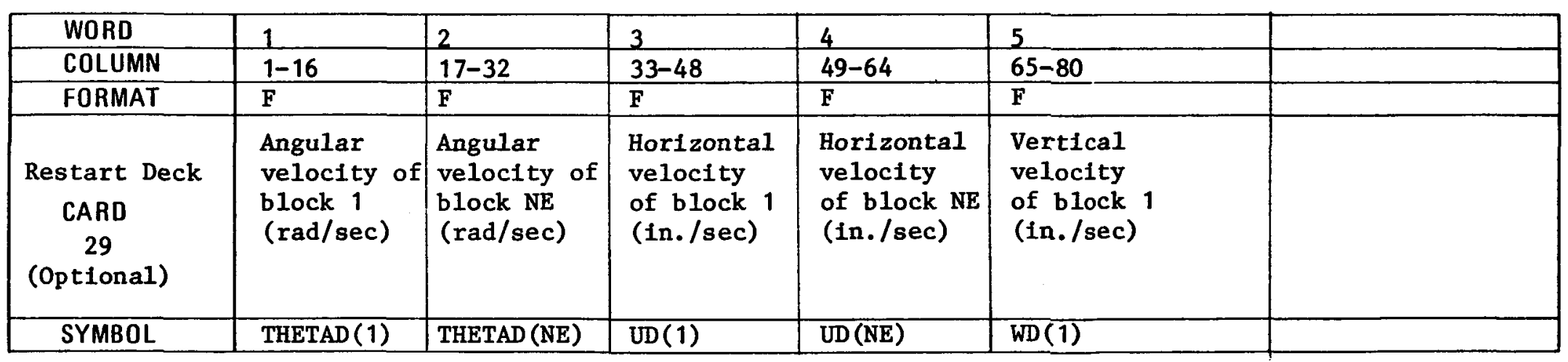

Enter NE values of THETAD followe by $N E$ values of UD followed by NE values of WD. Us as many cards as required.
Enter NE values of THETA followed by $N E$ values of $U$ followed by NE

values of $W$. Use as many cards as required.

required.




\begin{tabular}{|c|l|l|l|l|}
\hline WORD & 1 & 2 & 3 & 4 \\
\hline COLUMN & $1-16$ & $17-32$ & $33-48$ & $49-64$ \\
\hline FORMAT & & & & \\
\hline $\begin{array}{c}\text { Restart Deck } \\
\text { CARD } \\
\text { (Optiona1) }\end{array}$ & $\begin{array}{l}\text { PCRV } \\
\text { horizontal } \\
\text { displacement } \\
\text { (in.) }\end{array}$ & $\begin{array}{l}\text { PCRV } \\
\text { horizontal } \\
\text { velocity } \\
\text { (in./sec) }\end{array}$ & $\begin{array}{l}\text { PCRV } \\
\text { vertical } \\
\text { displacement } \\
(\text { in.) }\end{array}$ & $\begin{array}{l}\text { PCRV } \\
\text { vertical } \\
\text { velocity } \\
(\text { in./sec) }\end{array}$ \\
\hline SYMBOL & UO & UOD & WO & WOD \\
\hline
\end{tabular}

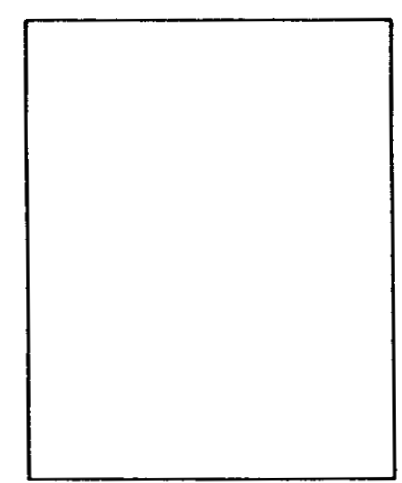

\begin{tabular}{|l|l|}
\hline WORD & \\
\hline COLUMN & \\
\hline FORMAT & \\
\hline & \\
\hline & \\
\hline & \\
& \\
& \\
& \\
\hline
\end{tabular}

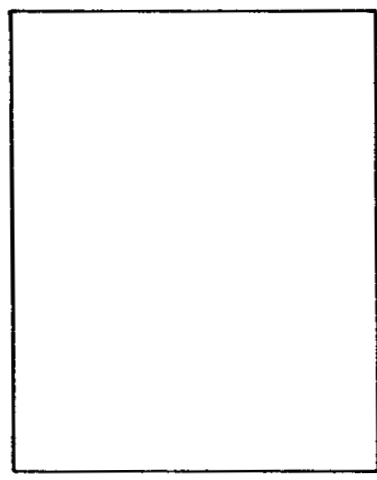

\begin{tabular}{|l|l|}
\hline WORD & \\
\hline COLUMN & \\
\hline FORMAT & \\
\hline & \\
CARD & \\
& \\
\hline SYMBOL & \\
\hline
\end{tabular}

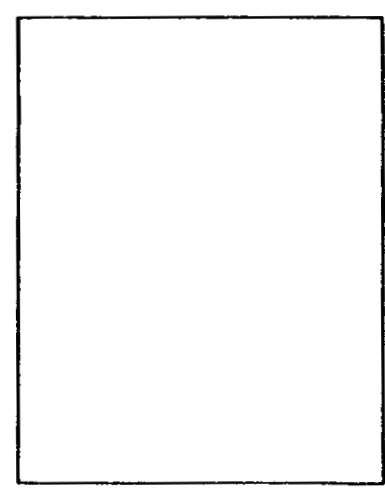


APPENDIX B

SAMPLE PROBLEM OUTPUT

B-1 


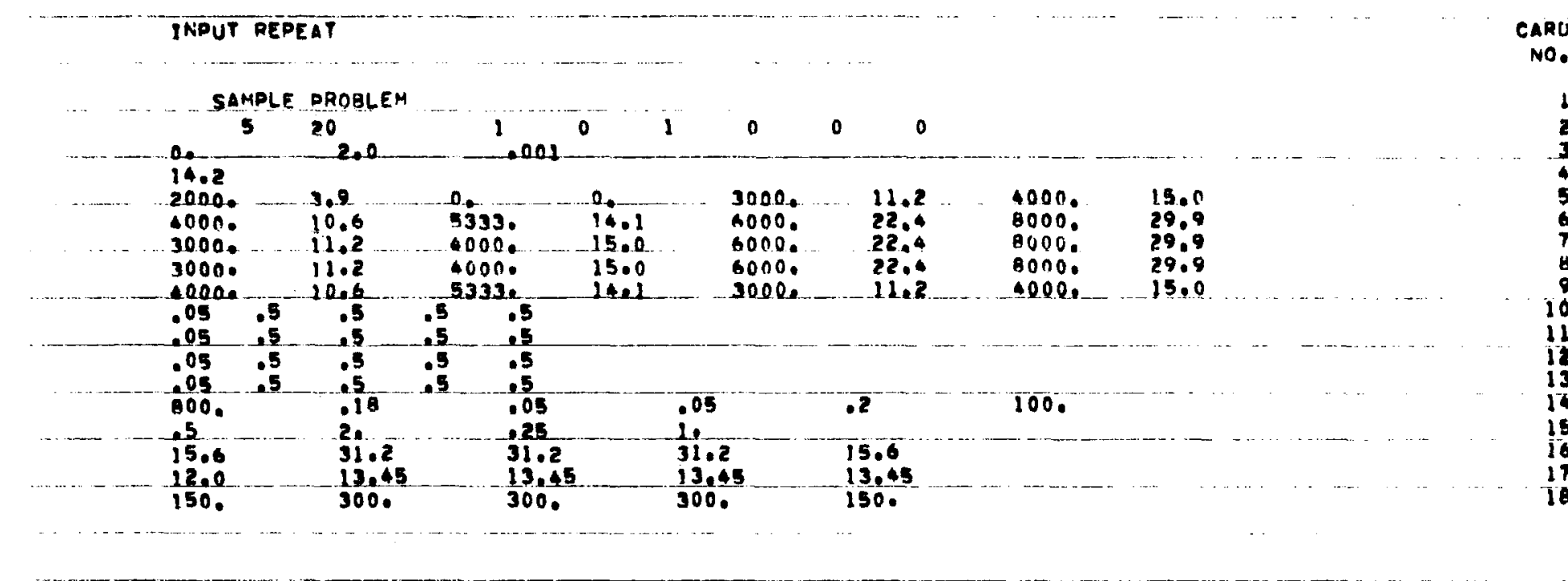

$\stackrel{W}{N}$ 
SAMPLE PaOalem

PROALEM START TIMEE

PROQLEM END TIMER

INTEQRATION TIME STEPA.001000

CENTRAL DIFFERENCE INTEGRATION

NUMBER OF ELEMENTSE
NUMBER OF EOUATIONS

GOUNDARY EXCITATION IN HORIZ.OIRECTION

EXCITATION FREOUENCY - $2.00 \mathrm{HZ}$

ACCELERATION AMPLITUDE OF EXEITATION .50006

GOUNDARY EXCTTATION IN VERT. DIRECTION

EXCITATION PREOUENCY $1.00 \mathrm{~Hz}$

ACCELERATION AMPLIIUDE OF EXCITATION $=.2500$

G

DISPLACEMENT AMPLTTUDE OF EXCITATION - -1.223 INCH

VELOCIIY AMPLITUDE OF EXCITATION - -15.37. INCH PER SEC

IN VERTICAL OIAECTION

DISPLACEMENT AMPLITUDE OF EXCITATION -2.447 INCM

VELOCITY AMPLITUDE OF EXCITATION - -15.374 INCH PER SEC

BLOCK GEOMETRIC WINTH, BOE 24.20

ELEMENT HETOAT, HTT

$15.60 \quad 31.20 \quad 31.20 \quad 31.20 \quad 15.60$

ELEMENT ROCKINO WIDTH, B(I)

$12.00 \quad 13.45 \quad 13.45 \quad 13.45 \quad 13.45$

ELERENT WETOHTS WFIT

$150.00 \quad 300.00 \quad 300.00 \quad 300.00 \quad 150.00$

ELEMENT MASS MATRIX

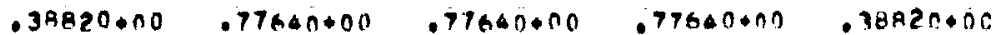

ROTARY INEAYIA MATRIX 


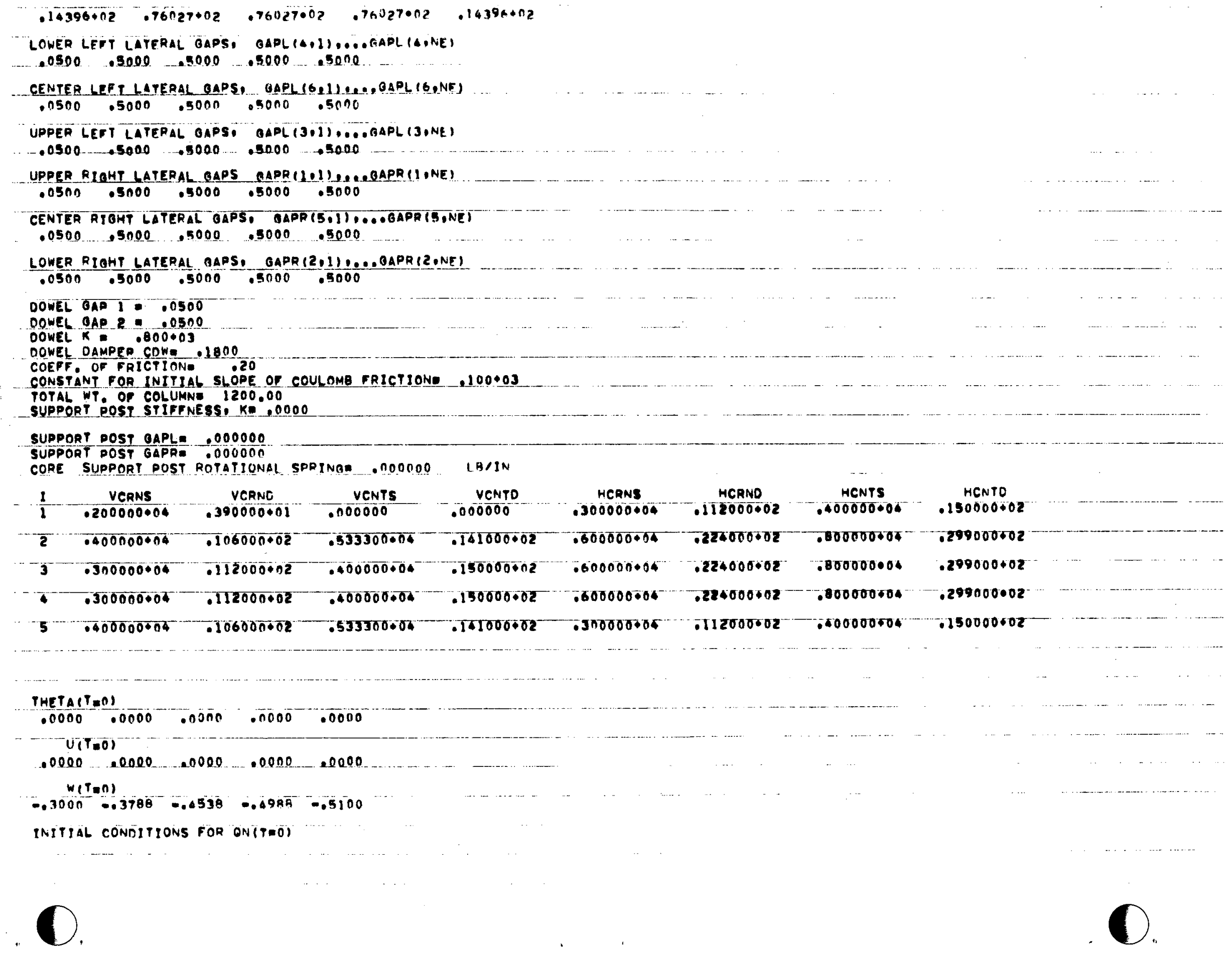




\begin{tabular}{|c|c|c|c|c|c|c|c|c|c|}
\hline .000 & .000 & .000 & - non & .000 & .00 ? & $.00 n$ & .000 & .000 & .000 \\
\hline $\begin{array}{l}.300 \% \\
.000\end{array}$ & $\begin{array}{l}.03790 \\
.000\end{array}$ & $\begin{array}{c}. .4540 \\
.000\end{array}$ & $\begin{array}{l}=499+.00 \\
.000\end{array}$ & $\begin{array}{l}=.510+00 \\
.000\end{array}$ & $\begin{array}{l}.000 \\
.000\end{array}$ & $\begin{array}{l}.000 \\
.000\end{array}$ & $\begin{array}{l}.000 \\
.000\end{array}$ & $\begin{array}{l}.000 \\
.000\end{array}$ & $\begin{array}{l}\text { non } \\
0100\end{array}$ \\
\hline
\end{tabular}

落 
COCO SIMULATIONi

SAMPLE PROBLEM

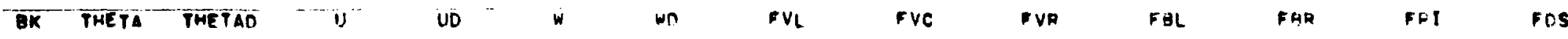

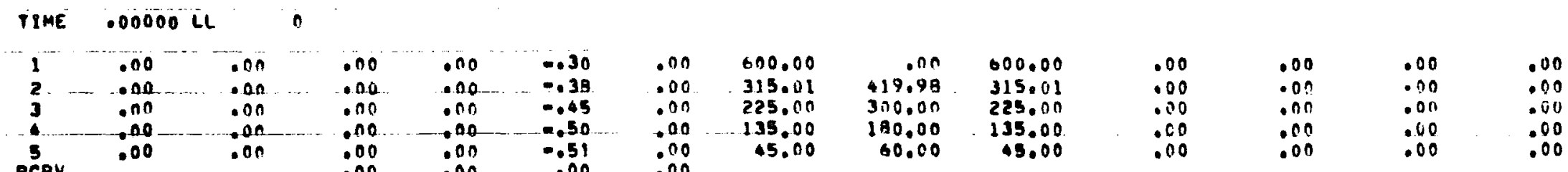

TIME .02000 L $\quad 20$

45.00

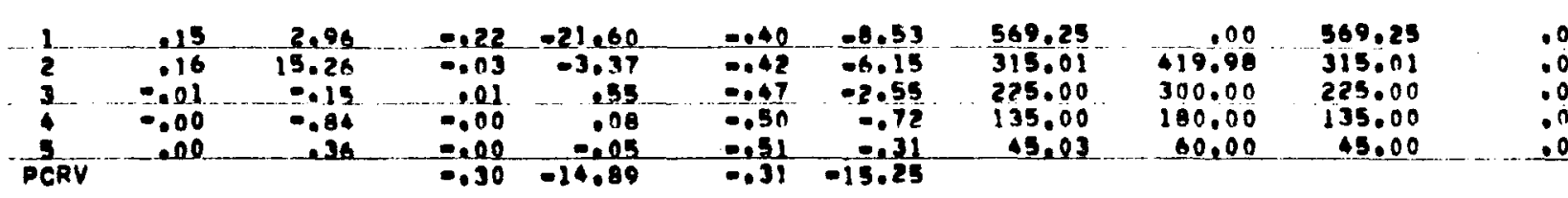

\begin{tabular}{rr}
.00 & 720.70 \\
.00 & .00 \\
.00 & .00 \\
.00 & .00 \\
.00 & .00 \\
\hline
\end{tabular}

$\begin{array}{rr}.00 & .00 \\ 182.09 & 59.66 \\ -43.09 & .00 \\ -20.23 & .00 \\ 5.12 & .00\end{array}$

TIME $.04000 \mathrm{LL} \quad 0$
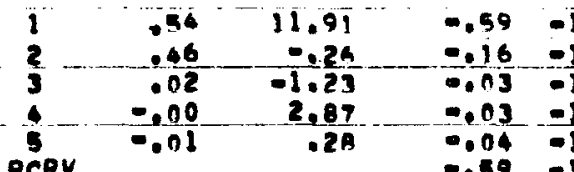

1.

TIME .0600O LL 60
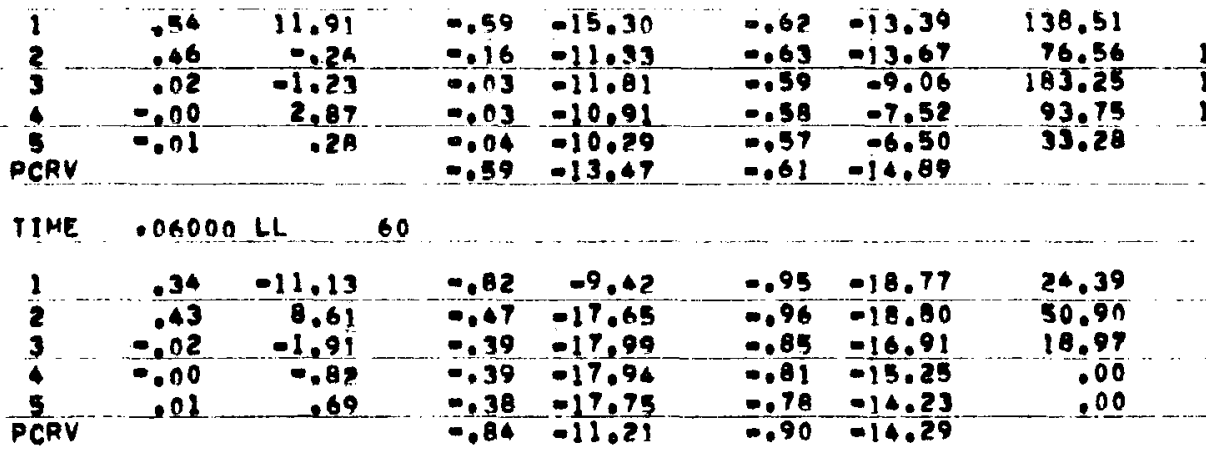<smiles>c1ccc2ccccc2c1</smiles>

$\begin{array}{rrrr}6.15 & 310.08 & .00 & .00 \\ .00 & 348.14 & 63.51 & 148.76 \\ .00 & 1112.76 & -19.88 & .00 \\ .00 & 1131.13 & -31.66 & .00 \\ .00 & 516.08 & 7.15 & .00\end{array}$

TIME $.08000 \mathrm{LL}$ Bo

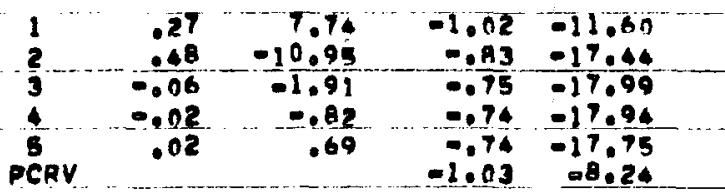
$0.07-17.65$ $=.95-18.77$

\section{0} $.39-17.90$ 24.39
50.90
18.97
.00
.00 .00
37.32
.00
.00
.00 166.62
04.02
.00
.00
.00 17.86
.00
.00 $\begin{array}{rr}.86 & 71.16 \\ .00 & 352.01 \\ .00 & 113.29 \\ .00 & 11.79 .43 \\ .00 & 531.09\end{array}$ $\begin{array}{rr}.00 & .00 \\ -23.90 & 157.76 \\ -3.79 & .00 \\ .00 & .00 \\ .00 & .00\end{array}$ TIME $\quad 10000 \mathrm{LL} \quad 100$

$$
0
$$

$\begin{array}{lll}-1.23-7.12 & -1.69-17.48 \\ -1.17-16.90 & -1.73-20.09\end{array}$

$1.17 \quad-16.99 \quad-1.73-21.29$

$1.10-17.04-1.74-30.71$

$1.19-17.75,-1.67=29.68$

$-1.16-0.75 \quad-1.46-12.44$

\begin{tabular}{rr}
240.92 & .00 \\
.00 & 139.50 \\
\hline .00 & .00 \\
.00 & .00 \\
.00 & .00
\end{tabular}

353.96
215.78 $\begin{array}{ll}3.94 & .00 \\ 5.78 & .00 \\ .00 & .00 \\ .00 & .00 \\ .00 & .00\end{array}$ 57.53
.00
.00
.00
.00 3
0
00
0
0 $\begin{array}{cc}.00 & .00 \\ -71.07 & 97.16 \\ .00 & .00 \\ .00 & .00 \\ .00 & .00\end{array}$

40.51
222.02
236.42
.00
.00
.00

471.10 148.43 270.35 $.0 n$ :0n 6.30
.00 .00
.00
.00
.00
.00
.01
.00
.00
.00
.00
.00 
COCO SIMULATION

SAMPLE PROGLEM

RK THETA TMETAD

TINE .12000 LL 120

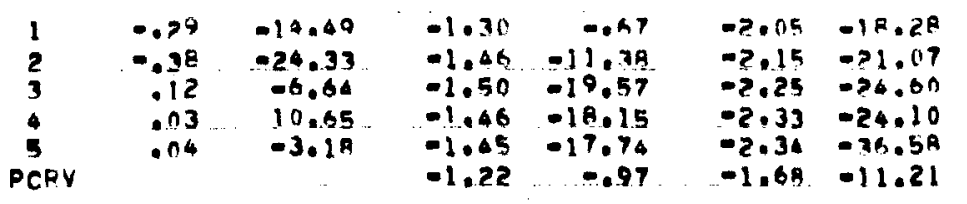

231.00

205.25

45.82

513.25

3.32 .63
$70.4 ?$

370.25
.77 .53

215.41

59.41

.jo
.0n
.on

.00

(a)

FI

fos

TIME . 16000 L6 . 140

\begin{tabular}{|c|c|c|c|c|c|c|}
\hline $\begin{array}{c}1 \\
2 \\
3 \\
4 \\
5 \\
\text { PCFV }\end{array}$ & $\begin{array}{r}-.65 \\
-.66 \\
-.09 \\
.03 \\
.11\end{array}$ & $\begin{array}{r}-12.76 \\
9.44 \\
-1.09 \\
-16.98 \\
-6.98\end{array}$ & $\begin{array}{l}-1.24 \\
-1.60 \\
-1.78 \\
-1.79 \\
=1.71 \\
-1.20\end{array}$ & $\begin{array}{r}2.45 \\
.23 \\
2.23 \\
-2.97 \\
-8.30 \\
2.91\end{array}$ & $\begin{array}{l}-2.42 \\
-2.57 \\
-2.72 \\
-2.81 \\
-2.89 \\
-1.80\end{array}$ & $\begin{array}{r}-18.26 \\
=20.21 \\
=21.34 \\
=>0.08 \\
=19.39 \\
=9.80\end{array}$ \\
\hline
\end{tabular}

$\begin{array}{rrrr}1202.81 & .00 & 932.10 & 335.30 \\ 617.59 & 730.13 & 539.19 & 449.17 \\ 249.08 & 597.07 & 646.76 & 1551.73 \\ 271.61 & 396.02 & 364.75 & 1739.10 \\ 264.29 & 274.37 & 171.47 & 652.90\end{array}$

335.30
409.17
551.73
739.10
652.80
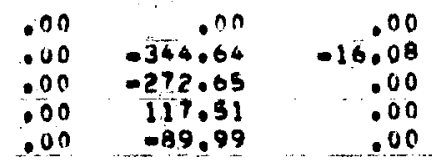

TIME $.16000 \mathrm{LL} 160$

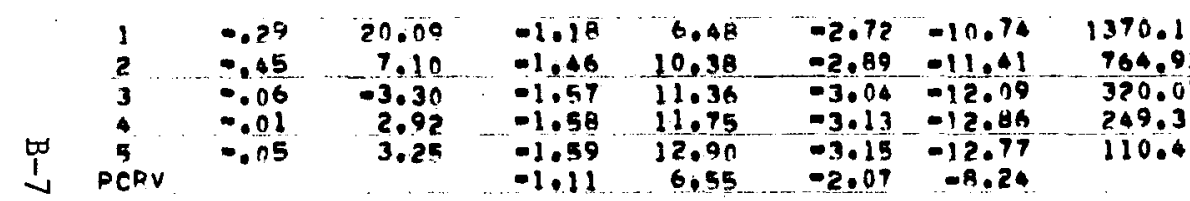

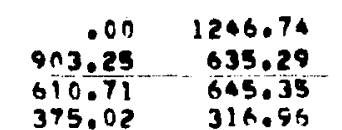

360.30
40.58
1525.26
1811.18
846.52

$\begin{array}{rr}.00 & -.00 \\ -344.04 & -50.41 \\ 66.42 & .00 \\ 112.05 & .00 \\ -34.21 & .00\end{array}$

TIME $18000 \mathrm{LL}$ IAO

$\begin{array}{rrr}1 & -.23 & 1.29 \\ 2 & -.29 & 5.40 \\ 3 & 0.17 & -4.94 \\ 4 & 0.03 & .79 \\ 5 & .108 & 5.22\end{array}$

$-1.00$

10.99

101.72

848.52

.00

34.2

.00

TIME .2N000 LL 200

$\begin{array}{ccccc}1 & -16 & 1.04 & -.77 & 12.71 \\ 2 & -.25 & .45 & -.97 & 12.77 \\ 3 & -.01 & .46 & -1.08 & 12.82 \\ 4 & .01 & -3.07 & -1.12 & 12.17\end{array}$

$\begin{array}{llll}.01 & -3.07 & -1.12 & 12.19 \\ .06 & -5.59 & -1.10 & 10.83\end{array}$

$\begin{array}{ll}-2.85 & -3.19\end{array}$

1370.60

.020 .01

1272.59

277.81

645.71

59.75

$\begin{array}{ll}420.95 & 389.59 \\ 143.69 & 129.03\end{array}$

.00
.00
.00

$\because 00$

.00

.00
.00
.00
00
.00

.00
-69.91
-17.07
47.41
28.71

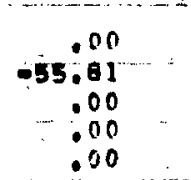

PCRV

$.72 \quad 12.46$

$-2.87$

$2.87 \quad .82 \quad 1302.50$

$.00 \quad 1207.10$

\subsection{4}

.04
.09
.00
.00

$.0 n$
.00
.00
.00
.00

.00
31.73
-23.81
-5.36

-5.36
-34.16

$-55.83$

$\begin{array}{rlll}-3.25 & 0.22 & 242.42 & 427.36\end{array}$

551.24

1.2. 11

.00

34.14

., $0 n$

.00

TIME . T2OOO LL 220

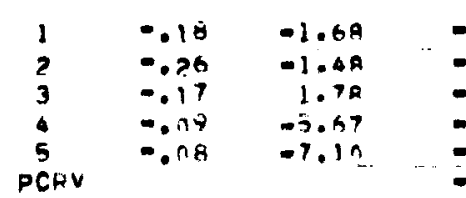

$\begin{array}{ll}-.50 & 14.00 \\ -.71 & 13.37 \\ -.81 & 13.39 \\ \because .87 & 12.44 \\ -.09 & 9.00 \\ -.45 & 14.00\end{array}$

$\begin{array}{lll}-2.81 \quad 0.65 \quad 1096.73 & 0.75\end{array}$

$-2.91 \quad 0.75 \quad 6.26 .09$

$3.0 ? \quad 9.04$

10.55

424.56

$200.4 ?$
09.97

$\begin{array}{rr}.00 & 1032.16 \\ 764.33 & 526.48 \\ 545.21 & 453.25 \\ 364.40 & 351.78 \\ 172.97 & 114.50\end{array}$

91.27
.00
.00
.110
.110

.00
.00
.00
.00

.00
26.27
-24.77
11.13
25.45

.00
-52.21
.00
.00
.60 
COCO SIMULATION

SAMPLE PAOBLEM

GX TMETA TMETAD

IIMF $24000 \mathrm{LL} 240$

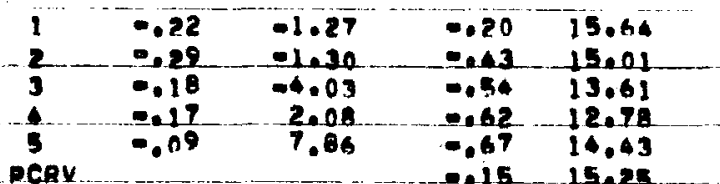

$\begin{array}{lll}-2.69 & 6.01 \quad 932.08\end{array}$ $0.06-10.26$ 10.26 102.96
49.89

TIME $2600046 \quad 260$
$0.12-535.00$

$6.00 \quad 167.32 \quad 205,86 \quad 221.46$

$9.20 \quad 100.73$

19.61

144.63

46.74

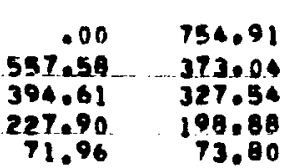

73,80
FBL

.00
.00

B. B
- 3 .

FRI N TOS 97

TIME.$\overline{28000 ~ L L ~} 280$

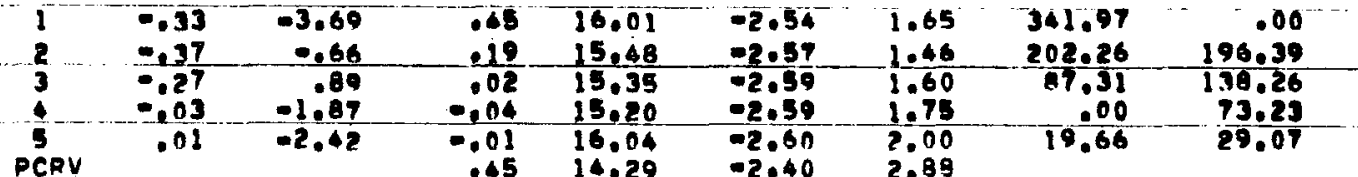

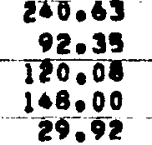

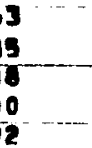

$\begin{array}{rr}72.59 & .00 \\ 56.00 & .00 \\ 15.99 & .00\end{array}$

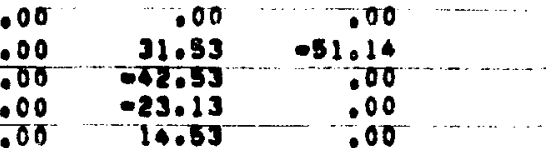

IIME $.30000 \angle L \quad 300$

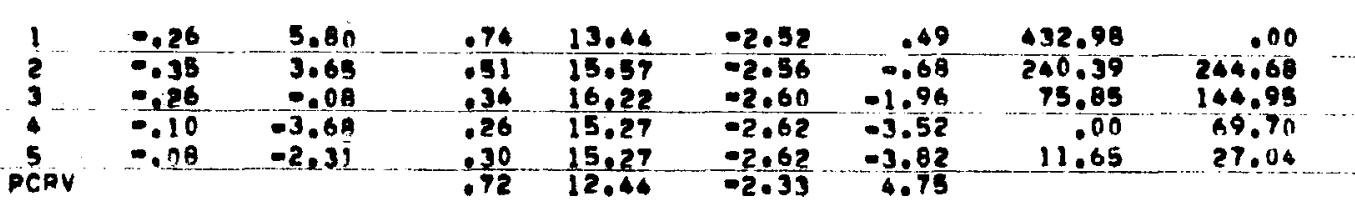

$\begin{array}{rrr}324.04 & .00 & 27.70 \\ 126.65 & .00 & .00 \\ 141.50 & .00 & .00 \\ 109.79 & 6.06 & .00 \\ 28.92 & .00 & .00\end{array}$

7.70
.00
.00
.00
.00

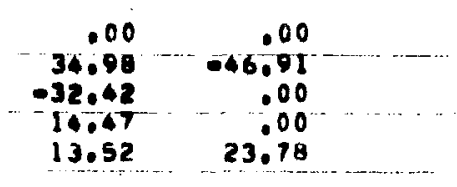

TIME $: 32000 \mathrm{LL} \quad 320$
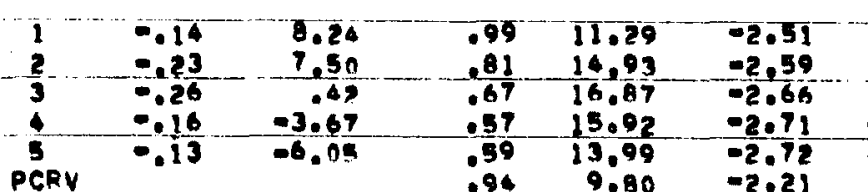

$-.03$

$676.01^{-}$

$.00^{-\cdots} 568.41$

$\frac{360.30}{232.35}-\frac{113.27}{203.79}-\frac{259.04}{258.33}$

332.35
$0.94 \quad 100.01 \quad 181.02 \quad 171.53$

$\frac{181.02}{63.46}$

$17 \frac{1}{6} \cdot 53$

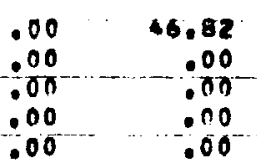

.00
+7.2
-33.0
-5.8
16.6

$\begin{array}{rr}.00 & .00 \\ .29 & -43.36 \\ .08 & .00 \\ 5.81 & .00 \\ .66 & 26.00\end{array}$

TIME .34000 LL 340

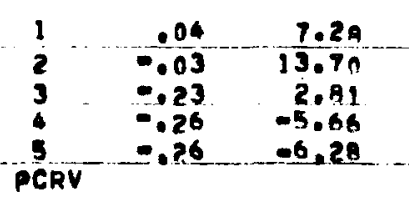

$\begin{array}{cc}1.18 & 6.94 \\ 1.09 & 12.01 \\ 1.01 & 19.23 \\ .90 & 16.30 \\ 1.97 & 13.67 \\ 1.11 & 6.35\end{array}$

-2.50
-2.62
-2.77
-2.79
-2.81

$1.56 \quad 866.05$

$-.19$

$-1.76$

391.69
204.58

607.007

429.16

252.05
184.37

PCRV

1.11 $-\frac{2.85}{1.24}$

$\begin{array}{ll}881.67 & 00 \\ 425.66 & 000 \\ 252.05 & .00 \\ 194.37 & .00 \\ 64.99 & .00\end{array}$

$\begin{array}{rr}.00 & 185.68 \\ .00 & .00 \\ .00 & .00 \\ .00 & .00 \\ .00 & .00\end{array}$

.00
12.0 .85
-25.86
-15.88
-23.55

.00
-36.00
.00
.00
25.12


TIME $.36000 \mathrm{LL} 360$

\begin{tabular}{rrrrrrrr}
1 & .21 & 11.35 & 1.27 & 2.77 & -2.43 & 6.06 & 1039.97 \\
2 & .28 & 15.57 & 1.32 & 9.94 & -2.57 & 4.94 & 528.99 \\
3 & 6.13 & 7.61 & 1.35 & 16.46 & -2.71 & 3.64 & 531.96 \\
4 & -.37 & -4.96 & 1.23 & 16.80 & -2.79 & 2.78 & 333.51 \\
\hline 3 & -.39 & -7.03 & 1.14 & 14.12 & -2.81 & 2.53 & 93.88
\end{tabular}

$\begin{array}{rrrr}.00 & 1126.40 & .00 & 206.90 \\ 763.28 & 616.00 & .00 & .00 \\ 546.84 & 268.32 & .00 & .00 \\ 329.52 & 182.90 & .00 & .00 \\ 111.22 & 73.03 & .00 & .00\end{array}$

.00
138.39
20.77
-38.08
-37.57

FDS

IIME .38000 LL 380

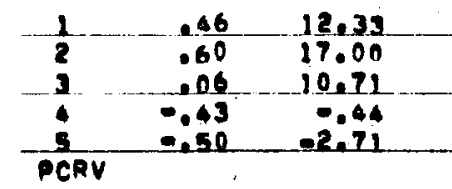

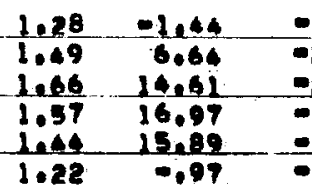

-2.25
-2.41
-2.96
-2.65
-2.68
-1.68

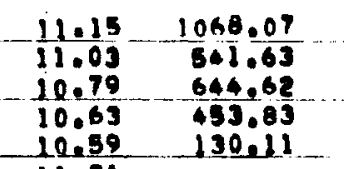

$.00 \quad 1250.37$

$823.62 \quad 706.67$

$374.12-188.65$

TIME $.40000 \mathrm{LL} \quad 400$

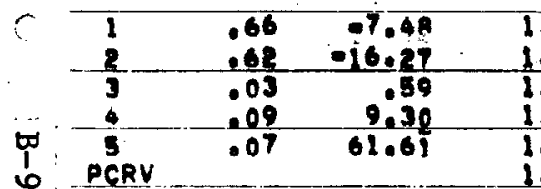

$\begin{array}{lllll}.23 & -3.17 & -1.98 & 15.79 & 1056.58\end{array}$ 126.62

$72.8 ?$

$\begin{array}{rr}.00 & 247.27 \\ .00 & .00 \\ .00 & .00 \\ .00 & .00 \\ .00 & .00\end{array}$

177.00

$-44.59$

$\begin{array}{r}-58.76 \\ \hline\end{array}$

$\begin{array}{r}.00 \\ -21.62 \\ .00 \\ 22.00 \\ \hline .10\end{array}$

TIME .42000 LL 420

\begin{tabular}{|c|c|c|c|c|c|c|c|c|c|}
\hline $\begin{array}{c}\frac{1}{2} \\
3 \\
4 \\
\frac{5}{\text { PCRY }}\end{array}$ & $\begin{array}{r}.27 \\
.46 \\
.12 \\
0.02 \\
.007\end{array}$ & $\begin{array}{r}-9.73 \\
-8.03 \\
9.20 \\
-6.99 \\
-16.79\end{array}$ & $\begin{array}{l}1.11 \\
1.34 \\
1.49 \\
1.51 \\
1.50 \\
1.03\end{array}$ & $\begin{array}{r}-9.17 \\
-11.15 \\
-10.61 \\
-10.25 \\
-14.68 \\
-9.94\end{array}$ & $\begin{array}{l}-1.62 \\
-1.96 \\
-1.86 \\
-1.93 \\
-1.94 \\
-1.18\end{array}$ & $\begin{array}{l}19.61 \\
21.85 \\
22.64 \\
23.80 \\
24.10 \\
13.47\end{array}$ & $\begin{array}{r}936.33 \\
565.17 \\
631.16 \\
261.02 \\
91.05\end{array}$ & $\begin{array}{r}.00 \\
769.35 \\
571.17 \\
347.36 \\
110.89\end{array}$ & $\begin{array}{r}1209.80 \\
604.60 \\
228.81 \\
282.67 \\
152.88\end{array}$ \\
\hline
\end{tabular}

TIME $.44000 \mathrm{LL}-640$

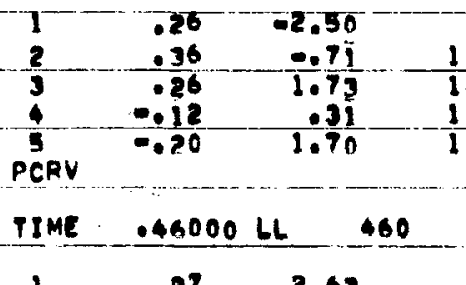

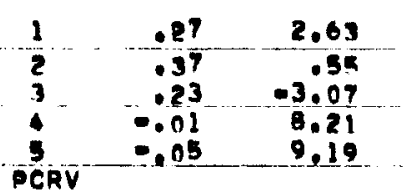

$\begin{array}{lllll}.63 & -13.35 & -.80 & 19.94 & 550.71 \\ .85 & -12.95 & -.85 & 21.36 & 264.23 \\ 1.02 & -12.09 & -.09 & 22.92 & 268.68 \\ 1.07 & -11.67 & -.92 & 23.90 & 278.79 \\ .98-11.36 & 0.93 & 24.20 & 00.17 \\ .59-13.67 & -061 & 14.89 & \end{array}$

\begin{tabular}{rr}
.00 & 658.98 \\
629.87 & 380.76 \\
316.22 & 205.66 \\
190.02 & 6.26 \\
62.20 & 13.13 \\
\hline
\end{tabular}

$\begin{array}{rr}.00 & 370.60 \\ .00 & 130.69 \\ .00 & 543.51 \\ .00 & 1213.08 \\ .00 & 926.74\end{array}$

\begin{tabular}{rr}
319.00 & .00 \\
-75.15 & 18.77 \\
-83.58 & .00 \\
55.45 & .00 \\
\hline
\end{tabular}
$.59-13.67-0.61-14.89$

816.50
367.06
51.93
290.75
99.46


530.71
264.23
268.68
278.79
90.17

-

928.23 $\begin{array}{rr}620.63 & 563.94 \\ 454.30 & 237.65\end{array}$ 454.30 90.28 191.41 $\begin{array}{rr}.00 & 299.03 \\ .00 & .00 \\ .00 & 15.91 \\ .00 & .00 \\ .00 & .00 \\ & \end{array}$

$\begin{array}{rr}93.72 & 27.68 \\ 45.73 & .00 \\ -19.76 & .00 \\ -60.40 & .00\end{array}$


COCO SIMULATION
SAMPLE PAOBLEM

BK THETA THETAO

TIME .49000 LL $\triangle B O$

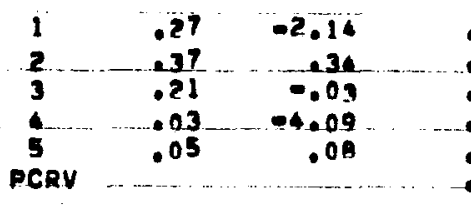

$.35-14,26$

$\begin{array}{lll}-14.02 & -.45 & 17.99\end{array}$

$\begin{array}{lll}-0.48 & 17.53\end{array}$

IIME .5000Q LL 500

$\begin{array}{lll}-14.67 & 0.49 & 19.21 \\ -10.02 & 0.49 & 19.23 \\ -14.09 & 0.31 & 15.25\end{array}$

134.64

185.59
155.57

155.57
41.25

255.42

102.04
32.50

20.0 .51

85.29
3.42
24.23

000.00

$.00 \quad 122.98$

122.98
.1 .0

45.76

27.01
16.44

.16 .25

30.82

. 00

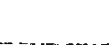

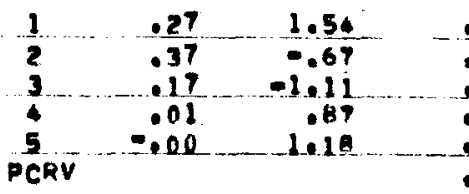

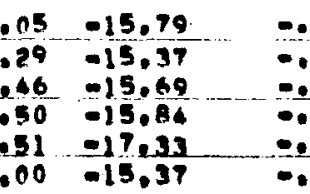

$\begin{array}{rrr}-.11 & 13.95 & 173.11 \\ 0.14 & 13.66 & 52.28 \\ . .16 & 13.61 & 132.59 \\ .017 & 13.33 & 98.04 \\ .17 & 13.30 & 19.13 \\ .170 & 15.39 & \end{array}$

\begin{tabular}{rr}
.00 & 297.11 \\
130.48 & 273.66 \\
96.64 & 15.26 \\
43.89 & .00 \\
10.26 & 27.36 \\
\hline
\end{tabular}

.00
.00
.00
.00
.00

110.93
.00
68.60
110.63

110.63
207.92

.00
0.25

32.50

11.19

9.12

TIME $.52000 \mathrm{LL}-520^{\circ}$

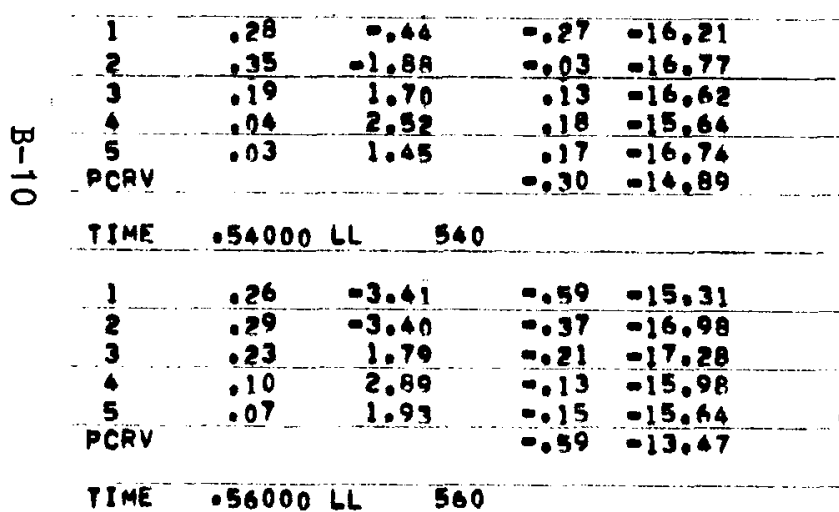

$14^{-} 11.49^{-} 274.94$

$.10 \quad 10.40$

119.5

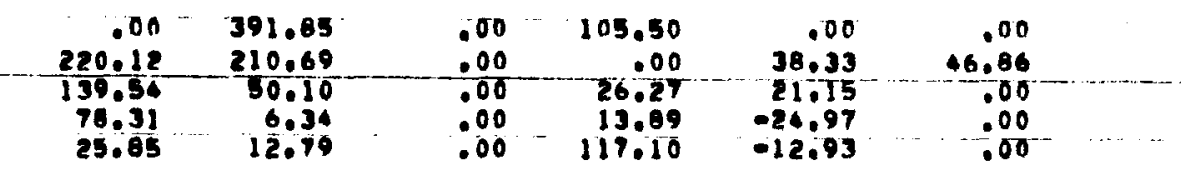

$31 \quad 15.25$

$\frac{111.12}{25.99}$

25.85

12.79

.00

$-12.93$

.00
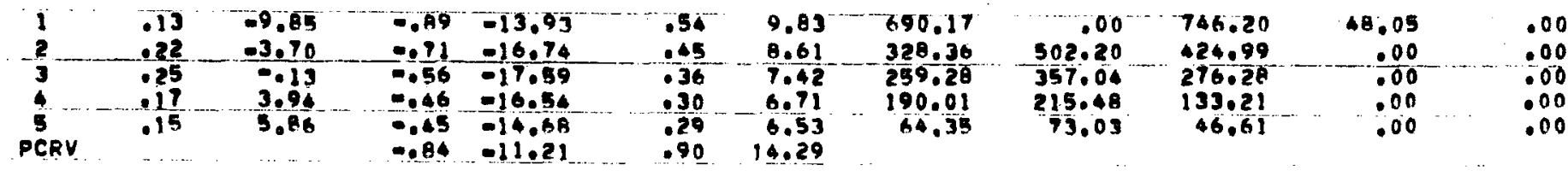

.00
.00
.00
.00
.00

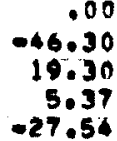

.00

.00
-9.37

TIME . \$9000 LL . SAO

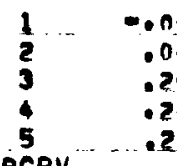

$$
\begin{array}{rr}
.04 & -6.29 \\
.04 & -14.04 \\
.24 & 0.75 \\
.26 & 5.49 \\
.26 & 5.36
\end{array}
$$

$\begin{array}{rrrrr}-1.10 & -8.00 & .75 & 11.50 & 856.92 \\ -1.02 & -14.16 & .64 & 10.90 & 417.14 \\ -.01 & -18.22 & .53 & 11.19 & 272.67 \\ -.79 & -16.75 & .47 & 9.69 & 194.27 \\ -.74 & 014.45 & .45 & 9.56 & 67.49 \\ -1.03 & -8.24 & 1.19 & 13.47 & \end{array}$

$\begin{array}{rr}.00 & 840.81 \\ 601.29 & 491.05 \\ 636.05 & 398.99 \\ 265.06 & 205.57 \\ 09.57 & 66.87\end{array}$

210.65
.00
.00
.00
.00

.00
.00
.10
.00
.00

$.0 n$
-129.20
35.49
17.41
7.39

37.28

.00

.000 
COCO SIMULATION

SAMPLE PROBLEM

BK THETA PAETAO

UID

wh

Fve

FV

r8L

FHK

FR!

os

TIME $.60000 \mathrm{LL} \quad 600$

$\begin{array}{ccc}1 & -.30 & -10.91 \\ 2 & -.26 & -13.62 \\ 3 & .16 & -7.67 \\ 4 & .38 & 5.55 \\ 5 & .39 & 7.96\end{array}$

$\begin{array}{lllll}-1.23 & -4.80 & 1.01 & 13.46 & 907.51\end{array}$

$-1.28-11.33 \quad \ldots 89 \quad 13.68 \quad 302.99$

$.77 \quad \frac{13.68}{13.84}-\frac{502.99}{252.14}$

$423.0 n$

$\$ 50.79 \quad 199.82$

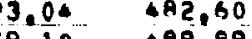

28) 91

489.8

289.19

.00
.00
.00

$\begin{array}{rrr}.00 & .01 & .00 \\ .00 & -134.18 & 19.70 \\ .00 & -19.78 & .00 \\ .00 & 41.78 & .00 \\ .00 & 22.46 & -0.49\end{array}$

TIME .62000 4 620

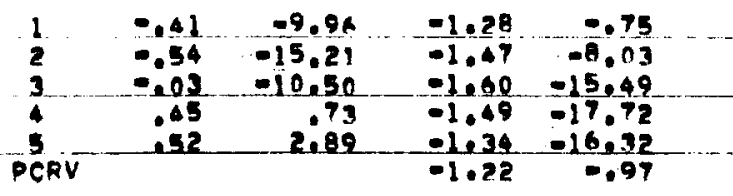

\begin{tabular}{rrr}
1.29 & 14.92 & 907.54 \\
1.19 & 15.87 & 504.06 \\
1.09 & 16.03 & 193.63 \\
1.02 & 17.47 & 127.30 \\
1.01 & 17.63 & 64.06 \\
\hline
\end{tabular}

0.00

819.51

252.16

$\begin{array}{rr}.16 & .00 \\ .00 & .00 \\ .00 & .00 \\ .00 & .00 \\ .00 & .00\end{array}$

\begin{tabular}{rr}
.00 & .00 \\
-178.20 & .00 \\
-45.82 & .00 \\
43.28 & .00 \\
43.70 & -0.32 \\
\hline
\end{tabular}

PIME .64000 LL 640

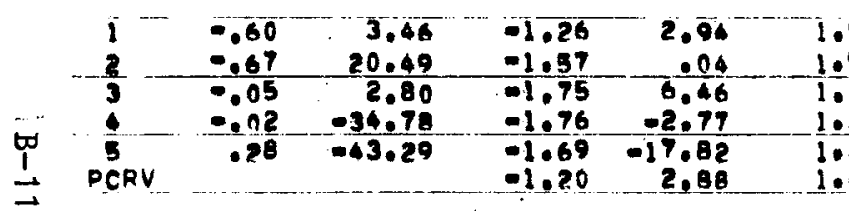

$.59-9.090^{\circ} \quad 06707$

$.00-671.37$

281.12

$543.47 \quad 321.68 \quad 317.70$

$02.15 \quad 486.53$

363,59

1177.57

231.56
70.79

.00
.00
.00
.00
.00

$\begin{array}{rr}.00 & .00 \\ -240.72 & 0.35 \\ -178.99 & .00 \\ 91.36 & .00 \\ 42.68 & .00\end{array}$

TIME .66000 LL 660

$\begin{array}{ccc}1 & -.25 & 11.38 \\ -2 & -.25 & 6.31 \\ 3 & -.08 & -1.91 \\ 4 & .00 & 5.79 \\ 5 & -.03 & 24.78\end{array}$

$-1.19$

$1.68 \quad 13.28$ 24.31

$81.62-168.10$

.......

TIME $.68000 \mathrm{LL} \quad 680$

$\begin{array}{ccc}1 & -.21 & -3.68 \\ 2 & -.31 & -2.26 \\ 3 & 0.12 & -2.73 \\ 4 & .11 & 4.76 \\ 3 & 019 & 2.72 \\ \text { PCRV } & & \\ \text { IIME } & .70000 \text { ㄴ․ } 700\end{array}$

$\begin{array}{ccc}1 & -.27 & -1.72 \\ 2 & -.37 & -3.39 \\ 3 & -.13 & 3.77 \\ 4 & .12 & -6.7 n \\ 5 & .14 & -5.86 \\ \text { PCRV } & -14 & \end{array}$

$\begin{array}{rl}-1.76 & 12.63 \\ -1.07 & 11.81 \\ -1.20 & 11.55 \\ -1.19 & 10.45 \\ -1.09 & 12.81 \\ -1.72 & 12.48\end{array}$

2.24
2.24
2.23
2.24
2.24
2.33

$\begin{array}{rr}4.80 & 244.64 \\ 5.01 & 158.61 \\ 5.98 & 10.60 \\ 6.53 & .00 \\ 6.60 & .00 \\ 4.75 & \end{array}$

$\begin{array}{rr}.00 & 193.69 \\ 138.20 & 48.70 \\ 101.60 & 141.80 \\ 46.85 & 119.09 \\ 12.10 & 37.86\end{array}$

314.22
-267.62

267.02
923.90

1334.37

$41.38-\frac{149.54}{254.63}$

$304.42 \quad 438.13$

$40.97 \quad 134.52$

872.55

.00
.00
.00
.00
.00

.00

$-205.70^{-0101.28}$

$60.66 \quad .00$

$\begin{array}{rr}59.25 & .00 \\ .29 .25 & .00\end{array}$

$.07 \quad \frac{16.89}{8.26}$

91.21

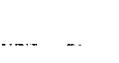

.00

319.64

341.21

220.08

.00
.00
.00

$107.92 \quad 121.07$

2.71

41.89

.00

.00

.00
.00
.00

.00

$\begin{array}{rr}.00 & .00 \\ -11.90 & -105.58 \\ -16.99 & .00 \\ 22.55 & .00 \\ -20.02 & .00\end{array}$

$.04 \quad 12.78$

$38.75 \quad 0.6$

.............

164.13
.00
99.87
39.29
.00

.00
.00
.00
.00
.00

.00
35.19
-35.22
-21.99
8.01

.00
-105.12
.00
2.00
2.52 
COCO SIMILATION!

SAMPLE PROGLEM

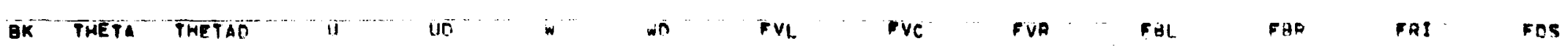

TIME $.72000 \mathrm{LL}^{-} 120^{-}$

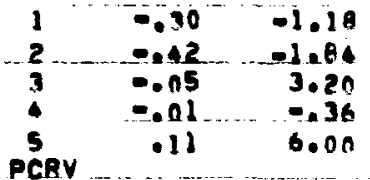

$2.31 \quad 1.00 \quad 249.15$

$.0 n$

121.44

$112.45 \quad .00$

36.61

21.37

117.96

96.67

57.97

160.02

.00

.00
.00
.00

33.09

33.09
-29.80

$-10.60$

6.33

.00

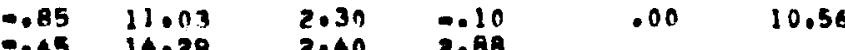

$21 \cdot 11$

$\begin{array}{rr}.00 & 218.17 \\ 193.94 & 00.93\end{array}$

133.52

$\begin{array}{ccc}1 & -.02 & -.28 \\ 2 & 0.02 & 2.11 \\ 3 & -.03 & -.01 \\ 0 & .01 & .00\end{array}$

$\begin{array}{lllll}-.20 & 15.23 & 2.30 & -2.19 & 351.16\end{array}$

$\begin{array}{llllll}0.53 & 15.60 & 2.26 & -3.20 & 210.00 & 193.94\end{array}$

$\begin{aligned} & 0.65 \\ & 0.65\end{aligned} \frac{15.63}{15.47}-\frac{2.24}{2.22}-\frac{5.08}{-5.53}-37.00$

69.28

217.51
65.93

63.00

171.41

35.85

10.35

.00

$\begin{array}{rr}.00 & .00 \\ -17.50 & -98.15 \\ -17.56 & .00 \\ 18.33 & .00 \\ -7.17 & 34.58\end{array}$

TIME - .76000 LL '

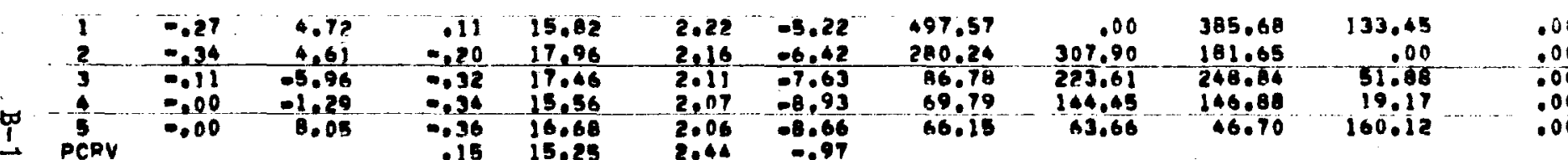

00
00
00
00
00

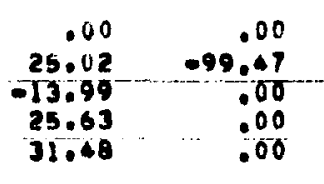

TIME . $18000 \mathrm{LL} \quad 780$

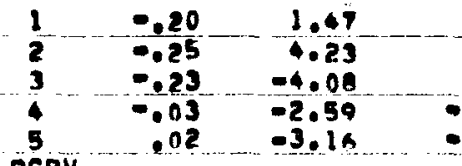

$\begin{array}{lllll}03 & 15.99 \quad 2.10 \quad-6.76 \quad 654.37\end{array}$

$.17-\frac{18.99}{18.30}-\frac{2.10}{2.02}-\frac{-6.76}{-7.75}-354.07-05-00.00-570.36$

$\begin{array}{llllll}10.07 & 1.94 & -8.52 & 226.16 & 310.18 & 248.52\end{array}$

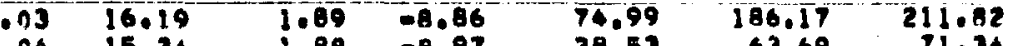

63.69

11.34

92.96

$92.96-.00$

23.75

87.04

$05-14.29-2.40 \quad-6.98$

.00

TIME $.80000 \mathrm{LL}$ 600

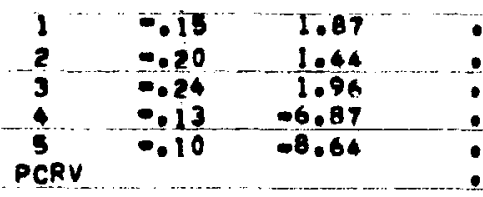

\begin{tabular}{lllllll}
75 & 16.17 & 1.97 & -6.20 & 753.49 & .00 & 691.26 \\
53 & 17.63 & 1.67 & -6.68 & 413.23 & 508.89 & 350.14 \\
40 & 18.61 & 1.78 & -7.29 & 287.73 & 363.51 & 257.54 \\
31 & 17.02 & 1.72 & -7.69 & 124.68 & 216.36 & 217.25 \\
\hline & 14.11 & 1.71 & -7.81 & 40.49 & 13.22 & 71.44
\end{tabular}

.00
.00
.00
.00
.00

.00
.00
.00
.00
.00

.00

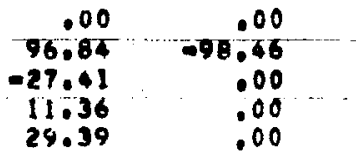

TIME .82000 LL 820

1

$\begin{array}{rr}.02 & 7.29 \\ \because .10 & 12.5 \\ -.22 & -.22 \\ -.27 & -6.5 \\ -.29 & -8.4\end{array}$

$72 \quad 12.45$

$\begin{array}{lr}1.71 & -7.81 \\ 2.33 & -4.75\end{array}$

0.49

13.22

99.66

$-27.55$

$-9.41$

PCR

$\begin{array}{ccc}1.02 & 9.10 & 1.85 \\ .197 & 15.85 & 1.75 \\ .77 & 18.93 & 1.05 \\ .65 & 17.08 & 1.59 \\ .54 & 1.003 & 1.58 \\ .04 & 9.00 & 2.21\end{array}$

$-5.65 \quad 7 \times 0.29$

$\begin{array}{r}-5.45 \\ -5.36\end{array}-35.86$

$\begin{array}{ll}-5.36 & 330.20 \\ -5.38 & 198.82\end{array}$

$\begin{array}{rr}.00 & 733.86 \\ 526.10 & 353.68\end{array}$

$\begin{array}{ll}526.10 & 353.64 \\ 387.81 & 273.76\end{array}$

238.44200 .80

68.91

.00
.00
.00
.00
.00

233.98

.00
165.81
-31.67
-5.35
13.08

.00
-74.09
.00
.00
.00

D 
COCO SIMULATION

SAMPLE PROBLEM

BK THE TA THETAD

TIME $.80000 \mathrm{LL} \quad 800$

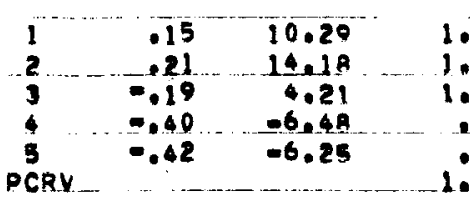

TIME .86000 LL 860

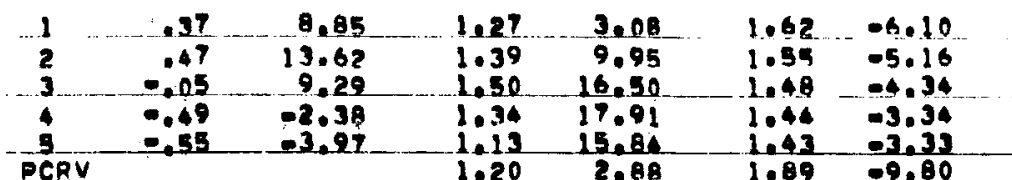

$617.36 \quad 000686.76$

$313.33 \quad 464.97 \quad 384.19$

$402.07 \quad 343.34$

38.19

209.83

78.01

$.0 n$
.00

.00
.00
.00

$190.00 \quad-31.00$

32.67

-49.59
-29.85

TIME $.88000 \mathrm{LL}-880$

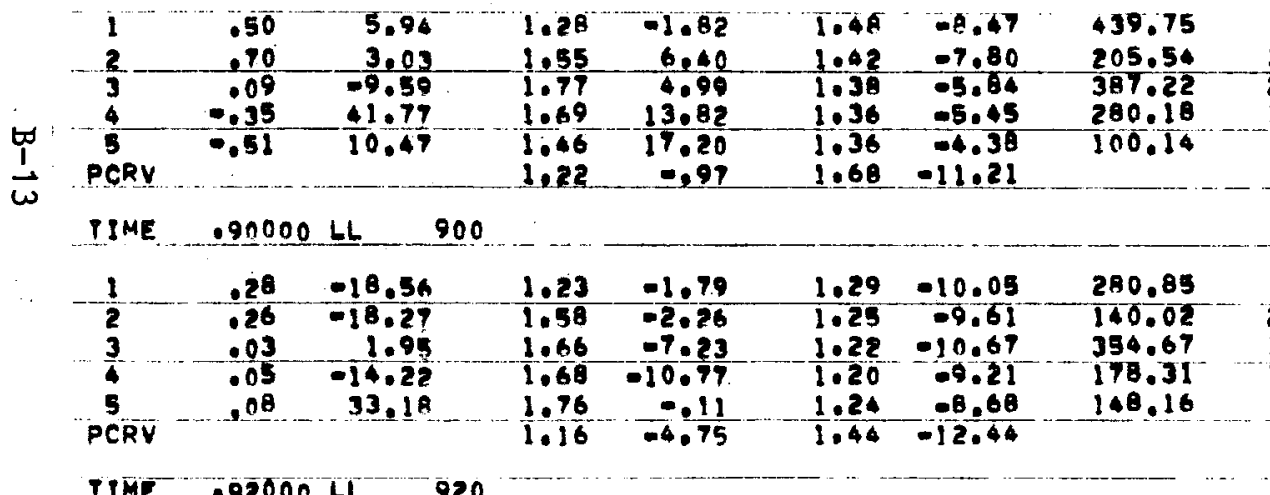

.00

$.00 \quad 267.48$

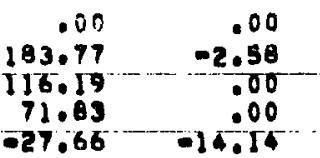

$\begin{array}{rrrr}268.84 & 16.04 & .00 & 1078.59 \\ 152.81 & 0.00 & .00 & 403.41 \\ 55.32 & 16.16 & .00 & .00\end{array}$

$.27 .66-14.14$

PIME $.90000 \mathrm{LL} \quad 900$

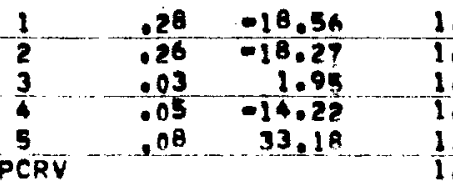

TIME $.92000 \mathrm{LL} \quad 920$

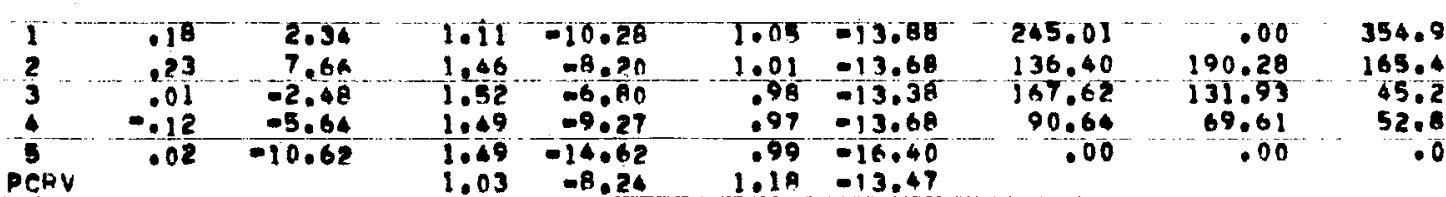

$\begin{array}{rrrr}.00 & 091.26 & .00 & 227.89 \\ 279.76 & 327.79 & .00 & 275.59 \\ 179.84 & 1.92 & .00 & 1220.53 \\ 106.22 & 113.18 & .00 & 1252.99 \\ 26.84 & .00 & .00 & 925.47\end{array}$

$\begin{array}{rr}.00 & .00 \\ 138.69 & 155.08 \\ -59.56 & .00 \\ 53.11 & 0.00 \\ -29.63 & -64.05\end{array}$

TIME $\quad 90000 \mathrm{LL} \quad 940$

$\begin{array}{rrrrrrrrrr}1 & .27 & 1.84 & .89 & -11.50 & .74 & -16.57 & 261.69 & .00 & 375.13 \\ 2 & .31 & -.62 & 1.26 & -11.96 & .70 & -17.19 & 128.56 & 212.76 & 190.61 \\ 3 & .02 & 1.61 & 1.35 & -11.52 & .67 & -18.50 & 207.41 & 140.77 & 7.30 \\ 4 & -.16 & 2.86 & 1.30 & -10.24 & .66 & -19.117 & 146.32 & 106.42 & 15.31 \\ 5 & -.31 & -8.60 & 1.21 & -13.59 & .63 & -17.69 & 101.15 & 71.37 & 53.21\end{array}$

7.30

33.21

.00
.00
.00
.00
.00

12.36
.00
2.16
299.01
956.42

.00
95.00
-51.76
-25.7

.00
172.60
.00

.00
13.06

$.00 \quad 13.06$

$\begin{array}{rr}.00 & 281.63 \\ .00 & 69.72 \\ .00 & 207.41 \\ 00 & 64.05 \\ .00 & .00\end{array}$

$\begin{array}{rr}.00 & .00 \\ -63.06 & 171.62 \\ 51.84 & .00 \\ -36.83 & .00 \\ -35.69 & .00\end{array}$ 
COCO SIMULATION

SAMPLE PRDQLEM

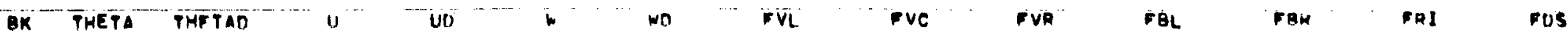

TIME $.96000 \mathrm{LL} \quad 960$

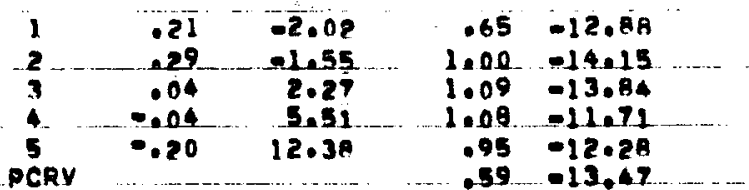

$.33-19.36 \quad 1140.07$ $-20.33 \ldots-196.12$

-65.80
16.83

$-6.45$

$-26.45$

.00

.00

$.35,89$

IIME . 98000 LL 980

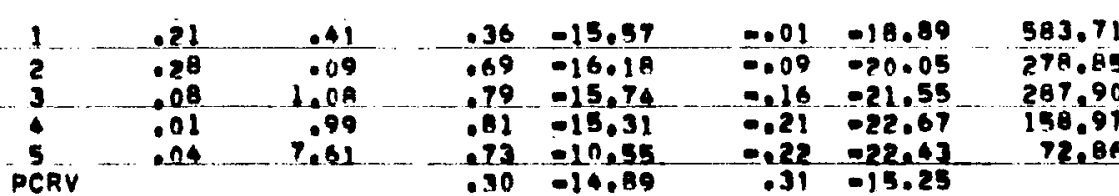

$\begin{array}{rr}.00 & 670.43 \\ 629.37 & 365.26 \\ 290.27 & 147.30 \\ 178.08 & 108.60 \\ 71.68 & 65.41\end{array}$

670.43
365.26
147.30
08.60

$.11-15.25$

$71.68 \quad 65.41$

.00
.00
.00
.00
.00

155.35

.00

66.31
137.69

.00

TIME $1000000 \mathrm{LL} \quad 1000$

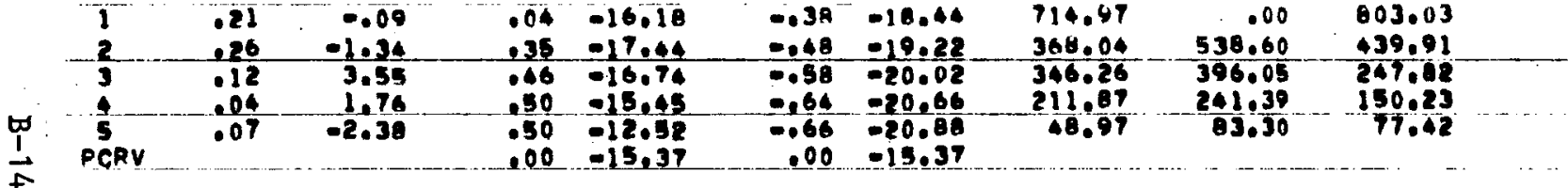

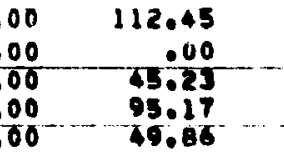

49.86

TIME $1.02000 \mathrm{LL} \quad 020$

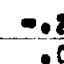

$-16.01$

$0.73-16.60 \quad 007.07$

$.00 \quad 891.81$

891.81

509.81

$433.75 \quad 313.05$

$83 . \%$

$-0.95034 .26$

250.34

08.60

155.68

$.19-16.08 \quad-1.04-17.24$

1.25

TIME $1004000 \mathrm{LL}^{-} 1040$

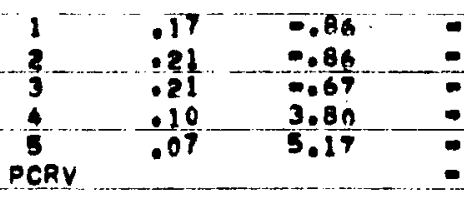

$660-15.06-1.06-14.00 \cdots-826.79$

$\begin{array}{rr}-.00 & 902.75 \\ 610.65 & 485.69 \\ 400.16 & 329.62 \\ 264.19 & 259.51 \\ 66.19 & 53.22\end{array}$

$\begin{array}{ll}.00 & .00 \\ .00 & .00 \\ .00 & .00 \\ .00 & .00 \\ .00 & .00\end{array}$

.00
.00
.00
.00
.00 $0.00 \quad 10.00$ $11.60-00$ $41.65-629.02$

TIME 1.00000 LL 1060

$\begin{array}{ccc}1 & 06 & -10.33 \\ 2 & 18 & -4.47 \\ 3 & 19 & -.15 \\ 4 & 19 & 4.47 \\ 5 & 19 & 5.69\end{array}$

$\begin{array}{lll}-.90 & -12.75 \quad-1.29-11.96\end{array}$

818.62

.00

480.25 136.61
.00
.00
.00
.00 .00
.00
.00
.00
.00

.00
-1690.44

21.67
-5.62

$-13.06$

263.86
98.09

125.25
64.06

$-42-14.79 \quad-1.59-10.28$

734.74

$-0.90-10^{-20}$

78.24

ค月.

.00

$\begin{array}{rr}109.17 & 113.76 \\ 17.86 & .00 \\ 2.79 & .00 \\ -26.71 & .00 \\ & \end{array}$


COCO SIMULATION

SAMPLE PROBLEM

BK THETA THETAO

u uñ

wo

FVL

FVC

Fv

Fàl

FR

$-10$.

TIME $1.00000 \mathrm{LL} 1080$

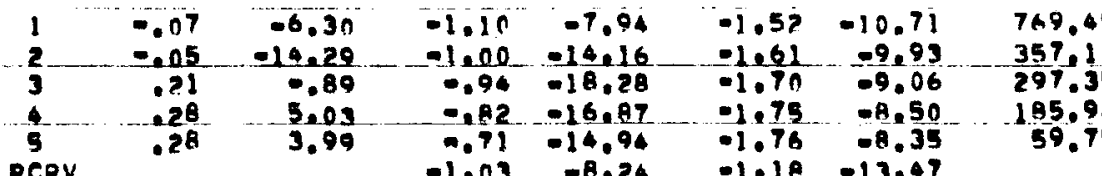

55.00

55.66

247.73

$\begin{array}{rr}791.19 & 187.63 \\ 474.64 & .00 \\ 351.73 & .00 \\ 186.06 & .00 \\ 64.27 & .00\end{array}$

.10
.00
.00
.00
.00

.00
-161.77
30.06
27.68
3.92

FI

Fos

IIME $1.10000 \mathrm{LL}$ i100

\begin{tabular}{|c|c|c|c|c|c|c|c|c|}
\hline $\begin{array}{r}1 \\
2 \\
-3 \\
5 \\
\end{array}$ & $\begin{array}{r}-.24 \\
-.31 \\
.11 \\
.38 \\
.10\end{array}$ & $\begin{array}{r}-9.76 \\
-12.09 \\
-7.59 \\
3.89 \\
6.89\end{array}$ & $\begin{array}{l}-1.23 \\
-1.26 \\
-1.30 \\
-1.16 \\
-1.01\end{array}$ & $\begin{array}{r}-5.01 \\
-11.07 \\
-17.01 \\
-17.62 \\
-15.40\end{array}$ & $\begin{array}{l}-1.73 \\
-1.80 \\
-1.87 \\
-1.91 \\
-1.93\end{array}$ & $\begin{array}{r}-10.14 \\
-9.52 \\
-8.87 \\
-8.46 \\
-8.37 \\
\end{array}$ & $\begin{array}{r}699.59 \\
358.87 \\
161.21 \\
126.29 \\
69.79\end{array}$ & $\begin{array}{r}.00 \\
474.73 \\
343.26 \\
206.66 \\
67.97\end{array}$ \\
\hline PCRY & & & -1.16 & -4.75 & -1.44 & -12.44 & & \\
\hline
\end{tabular}

\subsection{2 \\ 350.06 \\ 362.87
219.06}

207.17

.17
.00
.00
.00
.00

.00
.00
.00
.00
.00

$\begin{array}{rr}.00 & .00 \\ -183.76 & 36.45 \\ -20.29 & .00 \\ 11.30 & .00 \\ 22.040 & .00\end{array}$

TIME $1 . \overline{12000 \mathrm{LL}} 1120$

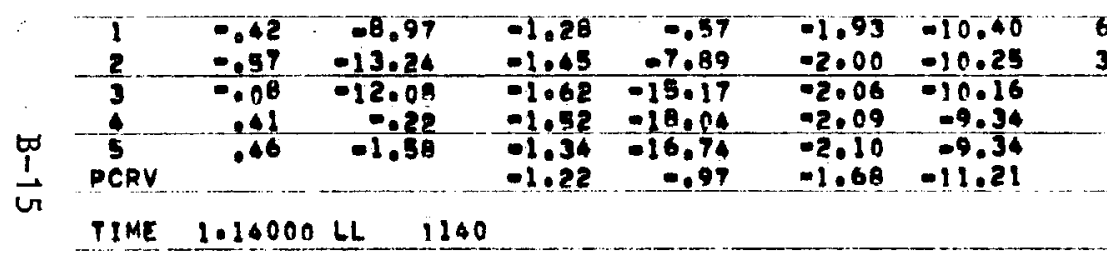

\begin{tabular}{|c|c|c|c|c|c|c|c|c|c|}
\hline $\begin{array}{c}1 \\
2 \\
3 \\
6 \\
5 \\
P C R V\end{array}$ & $\begin{array}{l}-.57 \\
0.67 \\
0.03 \\
0.08 \\
.02\end{array}$ & $\begin{array}{r}3.42 \\
21.47 \\
4.53 \\
-20.77 \\
-31.71\end{array}$ & $\begin{array}{l}-1.25 \\
-1.56 \\
-1.75 \\
-1.77 \\
-1.89 \\
-1.20\end{array}$ & $\begin{array}{r}3.24 \\
-1.12 \\
6.39 \\
1.42 \\
-18.39 \\
2.89\end{array}$ & $\begin{array}{l}-2.15 \\
-2.22 \\
-2.27 \\
-2.30 \\
-2.30 \\
-1.89\end{array}$ & $\begin{array}{l}-11.23 \\
-11.26 \\
-10.53 \\
-11.96 \\
-10.02 \\
-0.80\end{array}$ & $\begin{array}{r}640.21 \\
385.41 \\
17.38 \\
126.04 \\
18.61\end{array}$ & $\begin{array}{r}.00 \\
359.57 \\
252.34 \\
145.27 \\
53.39\end{array}$ & $\begin{array}{r}117.72 \\
191.42 \\
404.69 \\
275.56 \\
140.40\end{array}$ \\
\hline
\end{tabular}

259.18

242.41

1132.26

46.74

.00
.00

.00

PCRV

$-1.20-18.39-2.30-10.02$

TIME $1.16000 \mathrm{LL}$ il60

\begin{tabular}{ccc}
1 & .26 & 13.80 \\
2 & -.30 & 5.05 \\
\hline 3 & -.05 & -.05 \\
5 & .03 & 6.60 \\
5 & .00 & 23.36 \\
PCRV & & \\
TIME & 1.18000 LL 1180
\end{tabular}

\begin{tabular}{|c|c|c|c|c|c|c|c|c|}
\hline $\begin{array}{c}1 \\
2 \\
3 \\
4 \\
5 \\
P C R V\end{array}$ & $\begin{array}{r}-.23 \\
0.33 \\
-.88 \\
.16 \\
.21\end{array}$ & $\begin{array}{r}-5.34 \\
-4.16 \\
-1.50 \\
6.79 \\
3.53\end{array}$ & $\begin{array}{l}-1.01 \\
-1.32 \\
=1.42 \\
-1.39 \\
-1.34 \\
-.04\end{array}$ & $\begin{array}{r}11.96 \\
10.30 \\
9.19 \\
9.75 \\
12.36 \\
9.90\end{array}$ & $\begin{array}{l}-2.56 \\
-2.65 \\
-2.73 \\
-2.79 \\
-2.89 \\
-2.21\end{array}$ & $\begin{array}{r}-9.79 \\
-110.70 \\
-11.48 \\
-11.76 \\
-11.99 \\
-6.55\end{array}$ & $\begin{array}{r}733.05 \\
477.48 \\
174.56 \\
96.77 \\
60.46\end{array}$ & $\begin{array}{r}.00 \\
479.95 \\
390.23 \\
216.01 \\
75.02\end{array}$ \\
\hline
\end{tabular}

$\begin{array}{rrr}636.42 & 386.61 & .00 \\ 302.50 & 000 & .00 \\ 350.77 & 21.30 & .00 \\ 246.00 & .00 & .00 \\ 80.72 & 70.71 & .00\end{array}$

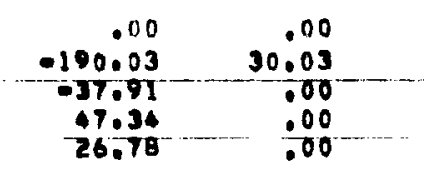

$\begin{array}{rr}.00 & .00 \\ -179.79 & .00 \\ -125.18 & .00 \\ -75.95 & .00 \\ 31.05 & 34.31\end{array}$

$1.19-16-2.36-10.0$

643.20

$.00-525.21$ $402.77 \quad 270.55$ \begin{tabular}{l}
275.39 \\
$155.47 \quad 139.35$ \\
\hline
\end{tabular}

$\begin{array}{ll}339.31 & .00 \\ 209.37 & .00 \\ 859.34 & .00 \\ 1355.48 & .00 \\ 890.45 & .00\end{array}$

$\begin{array}{rr}.00 & .00 \\ -190.97 & -110.58 \\ 50.29 & .00 \\ 66.37 & 0.00 \\ 31.90 & 10.05\end{array}$

$\begin{array}{rrr}.56 & 0.66 & -2.54-12.76\end{array}$ 38

0.05 
COCO SIMULATION

SAMPLE PROQLEM

- 11.

BE THETA THETAD

UD

wo

$F \sqrt{1}$

Fu

rve

$F B \bar{L}^{-}$

PBR

FRI

ros

TIME $1.20000 \mathrm{LL} 1200$

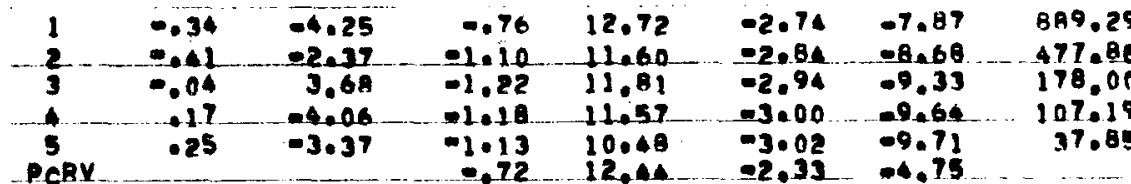

PIME 1.22000 LL 1220
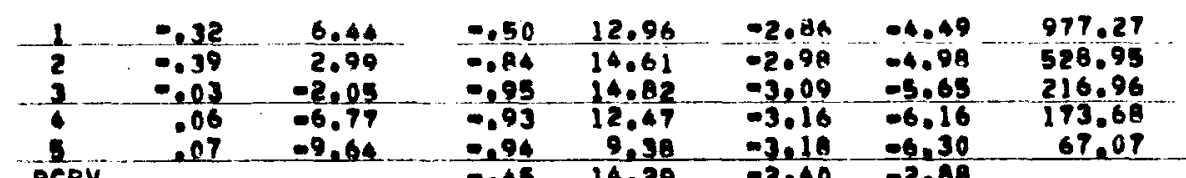

.00
640.021
457.53
274.58
92.29

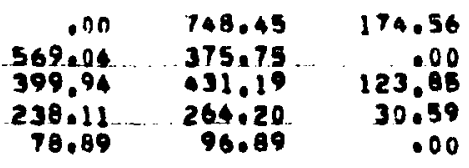

.00

$$
\begin{aligned}
& .00 \\
& .00 \\
& .00 \\
& 000 \\
& 000
\end{aligned}
$$

36.03

36.03
-45.20 $-22.72$ 39.44

.00

.00

.00

.00

044.03

134.40

$26.06-21.73$

$\begin{array}{r}1.73 \\ .00 \\ \hline\end{array}$

.00
.00
000
000
.00

$-10.00$

$-10.75$

$-12 \cdot 15$

39.67

TIME $1.24000 \mathrm{LL} 1260$

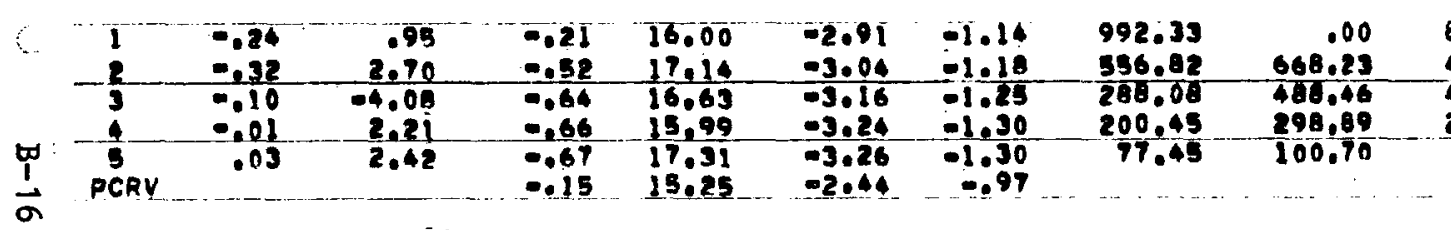

0907.71

469.43
250.00
90.92

0.00
141.04

$\frac{141.80}{191.40}$

30
.00
.00
.00
.00
.00 $\begin{array}{r}.00 \\ 27.85-111.90 \\ \hline\end{array}$ $-140.12 \quad .00$
-20.32

TIME $1.26000 \mathrm{LL} \quad 1260$

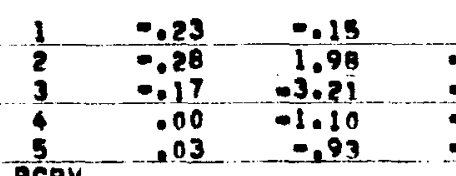

$.12 \quad 16.80 \quad-2.90$

2.31

992.28

533.90

668.00

804.15

137.99

4.41
275.87

275.87

298.95

90.77

137.99
67.00
93.57
120.36

$\begin{array}{rr}0.33 \quad 16.24 \quad-3.23 \quad 3.55 \\ .15-15.25 & -2.44\end{array}$

TIME I.2A0ON LL 1280

1
2
3
5
5

$\begin{array}{ll}0.21 & 2.62 \\ -.22 & 1.50 \\ 0.20 & 0.03 \\ -.06 & -0.49 \\ 0.02 & -5.03\end{array}$

$.45 \quad 16.32$

97

TIME 1.30000 LL . 1300

$\begin{array}{ccc}1 & -.12 & 9.01 \\ 2 & -.20 & 3.22 \\ 3 & -.20 & .25 \\ 5 & -.16 & -5.56 \\ 5 & -.16 & -7.18\end{array}$

$\begin{array}{lll}.77 & 14.81 & -2.69 \\ .55 & \frac{17.71}{-2.79} \\ .03 & 18.42 & -2.88 \\ .34 & 17.12 & -2.93 \\ .28 & \frac{14.65}{172} & -2.95 \\ 12.44 & -2.33\end{array}$

$\begin{array}{ll}7.45 & 072.17 \\ 8.21 & 463.63 \\ 0.97 & 303.57 \\ 9.43 & 154.04 \\ 9.54 & 53.76 \\ 4.75 & \end{array}$

.00
59.0 .46
422.36
253.94
94.15

413.11

329.94

240.67

Q4.15

79.91 
COCO SIMULATION

SAMPLE PAOBLEM

BK THETA TRETAO

TTME $1.32000 \mathrm{LL} 1320^{\circ}$

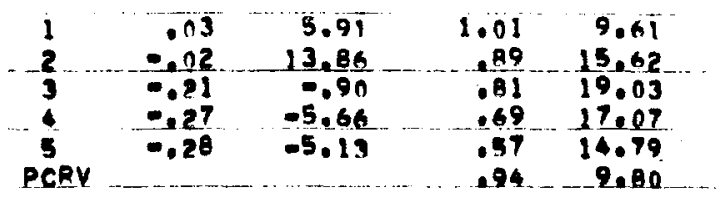
$-2.61 \quad 9.19 \quad 49.60$
-2.69 $\begin{array}{lll}-2.69 & 9.76 & 300.16 \\ -2.93 & 10.16 & 159.22\end{array}$ $\begin{array}{r}-2.93 \\ -2.74 \\ -2.70 .16 \\ 10.25\end{array}-\begin{array}{r}159.22 \\ 54.97\end{array}$ $-2,21$ 6.55 219.80 12.68

IIME 1030000 LL 1300

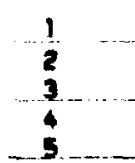<smiles>C1CCCCC1</smiles>

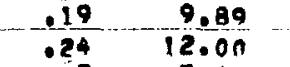
$0.17 \quad 5.62$ $\begin{array}{llll}0.39 & -5.27 & 1.18 & 18.0\end{array}$ $\begin{array}{r}-1.26 \\ \hline\end{array}$

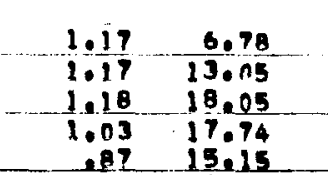

$$
\begin{aligned}
& -2.35 \\
& -2.42 \\
& -2.49 \\
& -2.53 \\
& -2.54
\end{aligned}
$$
........ -

TIME 1.36000 LL 1360

\subsection{5} 6.51 9,80 99.97 9.00 9.52 9.52 9.24

$\begin{array}{rr}.00 & 630.28 \\ +33.59 & 329.16 \\ 308.22 & 158.86 \\ 103.27 & 115.12 \\ 60.20 & 41.29\end{array}$
630.28
329.16
158.86
$i 15.12$
11.29 41.2 693.32
333.06
275.09
175.05 175.05
55.03
$-12$.

EAI Fos

$\begin{array}{rrrr}.00 & 172.19 & .00 & .00 \\ .00 & .00 & 145.87 & -75.16 \\ .00 & 000 & -26.47 & .00 \\ .00 & .00 & -17.11 & .00 \\ .00 & .00 & -4.37 & .00\end{array}$

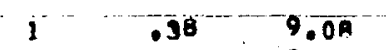

5.27

2.7

$.21-520.23$

$.0 n \quad 641.17$

$\begin{array}{lll}257.71 & 391.59 & 366.76\end{array}$

$276.91-60.17$ $\begin{array}{rrr}272.56 & 161.74 & 39.14 \\ 69.41 & 97.10 & 33.33\end{array}$

$\begin{array}{rr}.00 & 102.98 \\ .00 & .00 \\ .00 & .00 \\ .00 & .00 \\ .00 & .00\end{array}$

166.55

18.21

$-42.18$

$-16.07$

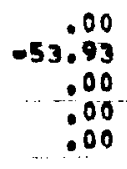

3.03
.00
.00
.00

tr

TIME $1.38000 \mathrm{LL} \quad 1380$

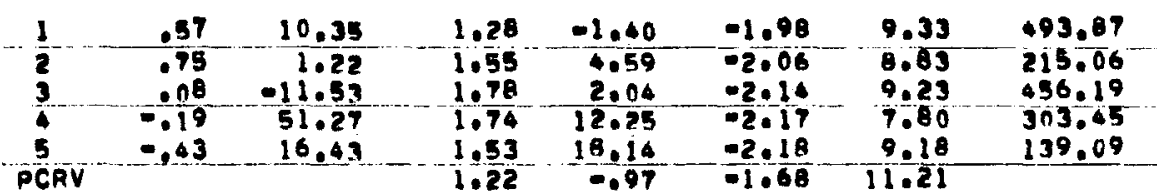

$\begin{array}{rrr}.00 & 733.43 & .00 \\ 425.63 & 35.27 & \\ 294.33 & 32.07 & .00 \\ 190.86 & 11.33 & 0\end{array}$

$.00 \quad 270.17$

197.69

1102.89

100.86
59.29

.00

.00

TIME I.4TOODO LL ITDO

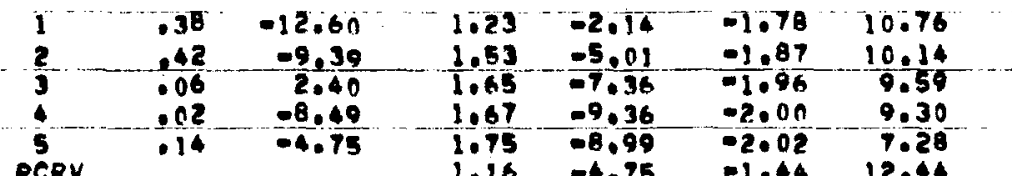

607.80 PCR $\begin{array}{llll}1.75 & -8.99 & -2.02 & 7.28 \\ 1.16 & -4.75 & -1.44 & 12.44\end{array}$

321.99

457.92
1.5 .79

$\begin{array}{rr}9.00 & 768.26 \\ 48.18 & 429.94 \\ 336.24 & 127.23 \\ 193.93 & 167.19 \\ 69.12 & 109.72\end{array}$

.00
.00
.00
.00
.00

280.91 244.81 1126.36 1267.45

TIME $1.42000 \mathrm{LL} 1420$

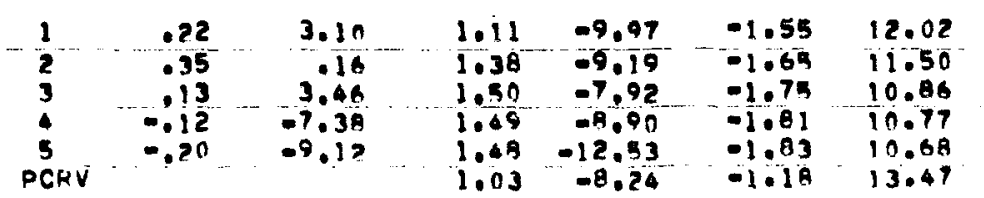

704.96 326.21 374.76
767.67 767.67
93.99

$\begin{array}{rr}.00 & 196.73 \\ 529.63 & 468.28 \\ 388.63 & 213.94 \\ 242.87 & 123.10 \\ 96.49 & 115.77\end{array}$

.00
.00
.00
.00
.00

335.00

21.04

14.30
783.25

$\begin{array}{rr}.00 & .00 \\ 145.26 & 78.69 \\ 28.90 & .00 \\ -83.61 & .00 \\ -49.25 & 6.56\end{array}$

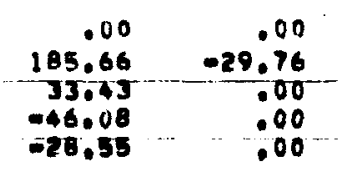

$\begin{array}{rr}.00 & .00 \\ 212.82 & -.57 \\ 128.16 & .00 \\ 67.18 & .00 \\ -35.90 & -34.037\end{array}$

$\begin{array}{rr}237.00 & .00 \\ -75.75 & 65.56 \\ -71.39 & .00 \\ -36.33 & -54.17\end{array}$


COCO SIMULATION

SAMPLE PROELEM

- 13.

BK THETA THETOD

UD

PVL FVE - FVR

FAL

FGP

FRI

Fos

TIME $1.44000 \mathrm{LL}$ i440

$\begin{array}{llllllll}-1 & .31 & 3.61 & .86 & -11.93 & -1.30 & 13.55 & 734.02\end{array}$

$\begin{array}{rrrr}1 & .31 & 3.81 & 1 \\ 2 & -.40 & 3.27 & 1 \\ 3 & .11 & -3.90 & 1 \\ 4 & . .16 & 2.64 & 1 \\ 5 & 0.23 & 3.53 & 1\end{array}$

$\begin{array}{lllll}.88 & -11.93 & -1.30 & 13.55 & 734.02 \\ 1.18 & -10.48 & -1.41 & 13.42 & 363.76 \\ .32 & -10.32 & -1.51 & 13.43 & 404.00\end{array}$

0.0020 .130 .03

.00
341.27
03.65
243.75

$8 \times 4.42$

223.96

27.41

.00
.00
.00
.00
.00

237.33

19.00
17.95

$\begin{array}{rr}.01 & .00 \\ 26.35 & 79.66 \\ 42.19 & .00 \\ 26.20 & .00 \\ -41.61 & .00\end{array}$

IIME 1.06000 LL 1660

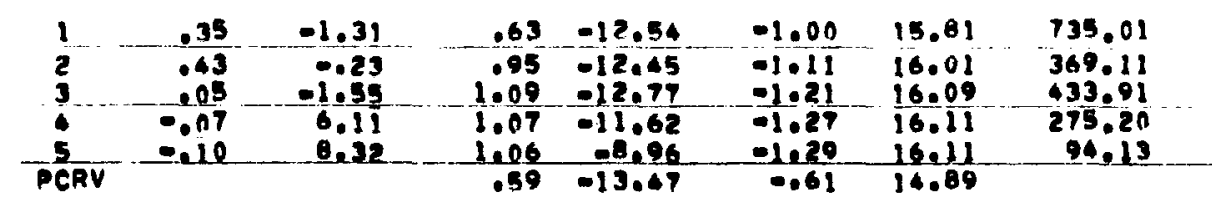

$\begin{array}{rrr}.00 & 875.95 & .00 \\ 564.91 & 479.24 & .00 \\ 03.36 & 197.97 & .00 \\ 63.62 & 136.08 & .00 \\ 81.28 & 40.70 & .00\end{array}$

.00
.00
.00
.00
.00

124.33
79.00
16.69
.00

\begin{tabular}{rr}
.00 & .00 \\
33.20 & 82.96 \\
32.00 & .00 \\
-8.64 & .00 \\
-40.63 & .00 \\
\hline
\end{tabular}

TIME T.48000 LL 1480

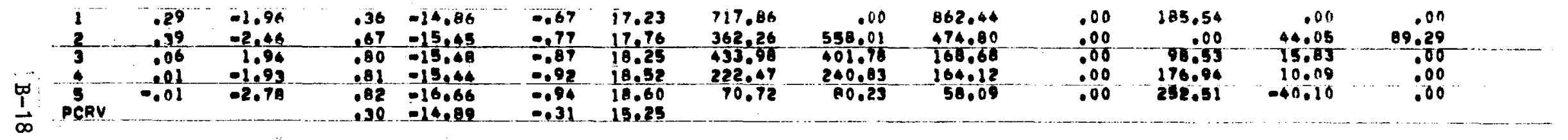

IIME 1.50000 LL 1500

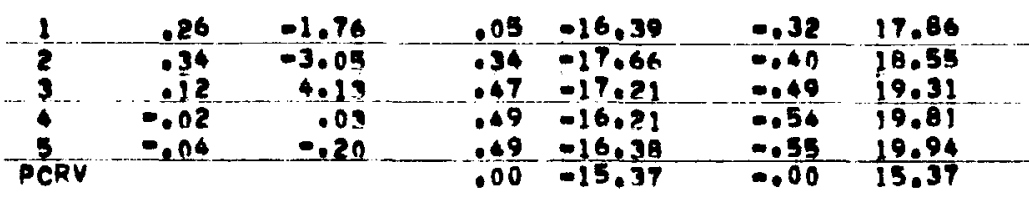

665.19

$326.47 \quad 513.00$

$.00 \quad 789.86$

787.86
166.77
168.45

195.26

377.63

$\begin{array}{r}230.02 \\ 71.07 \\ \hline\end{array}$

152.46

TIME $1.52000 \mathrm{LL} 1320$

$\begin{array}{ccc}1 & .23 & -1.32 \\ 2 & .27 & -3.42 \\ 3 & .20 & 2.90 \\ 4 & .01 & -3.21 \\ 5 & -.02 & 1.95 \\ \text { PCAV } & & \\ \text { TIME } & 1.54000 L L & 1540 \\ 1 & .17 & -3.35 \\ 2 & .22 & -2.59 \\ 3 & .22 & -.55 \\ 5 & 111 & 6.01 \\ \text { PCgY } & .08 & 7.03\end{array}$

$-.28^{-\cdots}-16.29$

50.15

$\because$

$.00 \quad 183.31$

\begin{tabular}{rr}
.00 & .00 \\
32.85 & 1.00 \\
15.06 & .00 \\
-12.96 & .00 \\
-33.02 & .00 \\
\hline
\end{tabular}

177.33

$-33.02$

$02-18.15 \quad 0.03 \quad 17.74-579.64 \quad 04.00 \quad 689.34$

$\frac{-18.15}{-18.0 ?}-\frac{0.03}{0.10} \frac{18.41}{19.08}-\frac{286.49}{319.46}-\frac{467.67}{324.24} \frac{395.05}{176.33}$

$.17-16.36$

$-014 \quad 19.47$

194.59
59.22

$\frac{195.11}{64.30}+\frac{98 \cdot 13}{37.24}$

$.31 \quad 15.25$

59.22

$\frac{98.13}{37.24}$

.00
.00
.00
.00
.00

09.74
.00
20.05
.00
.00

$\begin{array}{rr}.00 & .00 \\ -75.75 & 90.05 \\ 26.57 & .00 \\ -21.93 & .00 \\ -22.42 & .00\end{array}$

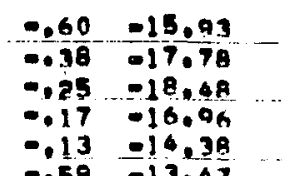

$\begin{array}{rrr}.39 & 16.62 & 684.79 \\ -0.32 & 17.09 & 249.46 \\ .027 & 17.56 & 223.54 \\ .23 & 17.86 & 185.28 \\ .22 & 17.90 & 55.79 \\ .61 & 14.60 & \end{array}$

.00
373.51
266.75
$158.5 \%$

581.39

310.85

177.06

A. 1.56

52.04

22.83

.00
.00
.00
.00
.00

1.87
.00
.00
.00
.00

.00
-80.24
24.55
4.23
-23.05

.00
01.58
.00
.00
.00

O 
COCO SIMULAIION

SAMPLE PROBLEM

- 14.

BK THETA THETAO

wo

$\overline{F V i}$

FVC FV FVR

PAL

FBA

FPI

Fus

TIME $1.56000 \mathrm{LL} 1560^{\circ}$

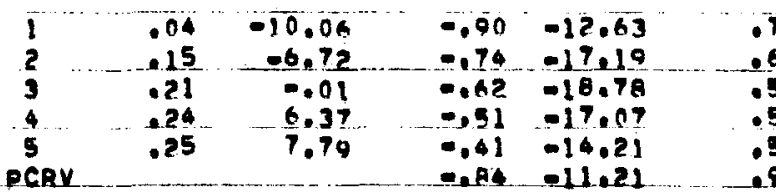

$15.9 n$

15.21

204.71

144.98

135.64

313.05

222,70
132,59

484.38

265.13

15.35

45.6

132,57
43,62

169.08

99.45
158.45
.00$$
\begin{array}{r}
.00 \\
.00 \\
.00
\end{array}
$$

$\begin{array}{rrr}.00 & .00 & .00 \\ .00 & -135.89 & 10.32 \\ .00 & 2.051 & .00 \\ .00 & 2.94 & .00 \\ .00 & -12.64 & .00\end{array}$

IIME $1.58000 \mathrm{LL}$ is80

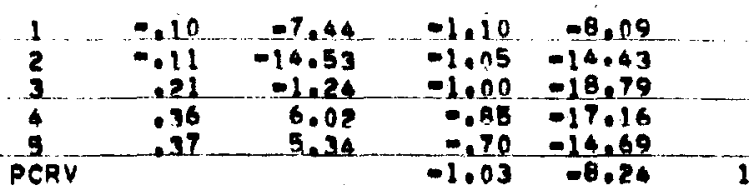

$.97 \quad 12.90$

431.67

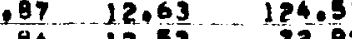
83 12.58 72.92
22,91

$\begin{array}{rr}.00 & 413.45 \\ 292.91 & 265.02 \\ 196.91 & 259.27 \\ 116.10 & 138.72 \\ 38.30 & 36.94\end{array}$

197.19
.00
.00
.00
.00

$\begin{array}{rrr}.00 & .00 & .00 \\ 000 & -140.71 & 57.07 \\ 000 & 26.07 & .00 \\ 000 & 33.42 & .00 \\ .00 & 10.06 & .00\end{array}$

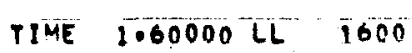

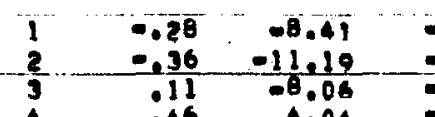

$$
\text { PCR }
$$$$
\begin{array}{r}
.046 \\
. .50
\end{array}
$$

$-1.23-6.77$

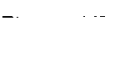

$339: 00$

258.76

423.22

423.22
182.17

362.14

288.88

250.43

0.43
.00
.00

52.67

62.11

.00

TIME T.64000 LE 1680

$\begin{array}{llr}1 & 0.48 & 13.49 \\ 2 & 0.48 & 24.08 \\ 3 & -.03 & -2.03 \\ -0.11 & -7.00 \\ \text { PCRV } & .21 & -35.57\end{array}$

$-1.26 \quad 2.02$

$\begin{array}{llll}1.60 & 9.03 & 671.70\end{array}$

.00

40.13

$269.58 \quad 273.18$

$6.56-1126.68$

$=1.73 \quad-.964$

$.53 \quad 0.96 \quad 388.9$

391.39

190,29

1222.66

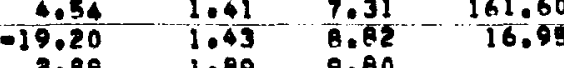

........

TIME 1.66000 LL 1660

$\begin{array}{crr}\frac{1}{2} & -.20 & 4.01 \\ 3 & -.31 & .03 \\ 4 & -.06 & -1.059 \\ 5 & .05 & 7.44 \\ \text { PCRV } & .02 & 25.18\end{array}$

$-1.197023$

$1.78 \quad 0.23$

668.24

9.39

0.45

373.2

133.19

414.32

300.0

193.45

539.54

362.24

1.58

7.50

113.79

94,30

17405

$\begin{array}{rr}19.71 & 1121.29 \\ & 923.13\end{array}$

$\begin{array}{ccc}.00 & .00 & .00 \\ .00 & -179.71 & .00 \\ .00 & -10.95 & .00 \\ .00 & 46.20 & .00 \\ .00 & 26.33 & .00\end{array}$

$1.11 \quad 0.55 \quad 2.07 \quad$ A.2

\begin{abstract}
...............
\end{abstract}


COCO SIMULATION

$-15$.

SAMPLE PRoblem

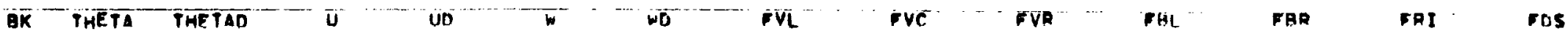

TIME $1.68000 \mathrm{LL} \quad 1660$

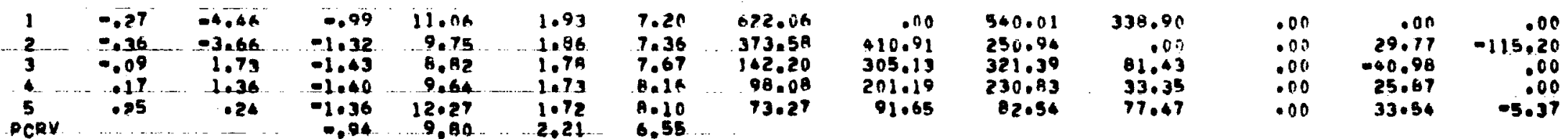

TIME 1.70000 LL ITPQ.

\begin{tabular}{|c|c|c|c|c|c|c|c|}
\hline $\begin{array}{ll}1 & 0.32 \\
2 & .00 \\
3 & 0.03 \\
4 & .10 \\
5 & .13\end{array}$ & $\begin{array}{r}-.36 \\
.09 \\
1.73 \\
-5.43 \\
-7.20\end{array}$ & $\begin{array}{r}-.77 \\
-1.11 \\
-1.22 \\
-1.19 \\
-1.13 \\
=.72\end{array}$ & $\begin{array}{l}11.63 \\
11.53 \\
11.94 \\
10.95 \\
10.93 \\
12.44\end{array}$ & $\begin{array}{l}2.06 \\
2.00 \\
1.93 \\
1.89 \\
1.88 \\
2.33\end{array}$ & $\begin{array}{l}6.27 \\
6.65 \\
7.08 \\
7.39 \\
9.47 \\
0.75\end{array}$ & $\begin{array}{r}620.92 \\
355.30 \\
127.47 \\
70.37 \\
24.56\end{array}$ & $\begin{array}{r}.00 \\
103.09 \\
299.08 \\
179.85 \\
59.62\end{array}$ \\
\hline
\end{tabular}

$\begin{array}{rrrrr}504.14 & 120.46 & .00 & .00 & .00 \\ 209.36 & .00 & .00 & 30.12 & -113.00 \\ 329.02 & 101.83 & .00 & -40.83 & .00 \\ 224.75 & 36.56 & .00 & 7.67 & .00 \\ 81.66 & .00 & .00 & 29.01 & 000\end{array}$

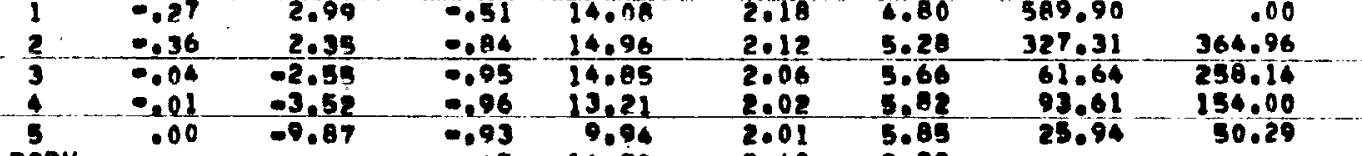

Pcav

$0.93 \quad 9.94$

$.01 \quad 5.85$

TIME $1.70000 \mathrm{LL} \quad 1740$

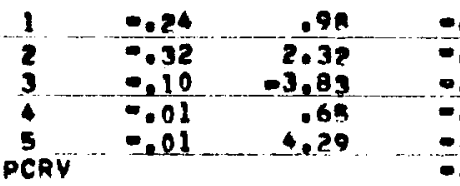

$-.20 \quad 16.19$

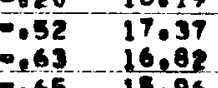

15.96

16.96

2

$29 \quad 2.37 \quad 906.28$

TIME $1.7 \overline{7000}$ LL 1760

$\frac{1}{3}$

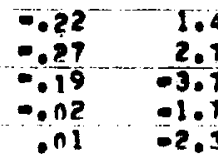

2.79
-3.79
-1.12

$+$

$.12 \quad 16.36$

6.36
8.14
7.61

16.15

15.92
15.25

2.27
$\frac{2.22}{2.16}$
2.16
2.15
2.15

.27
.22
.18
.16

TIME $1.78000 \mathrm{LL} \quad 1780$

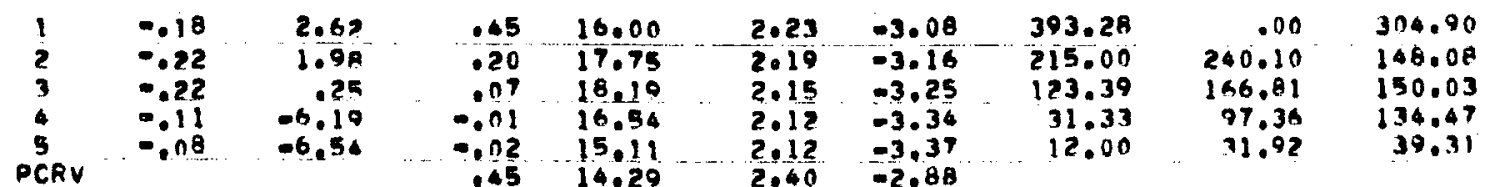

$2.40-2.80$

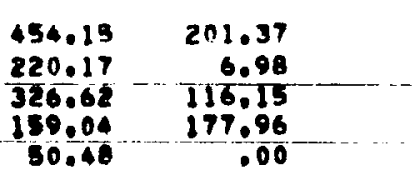

200.67
19.00
216.07
13.02

$\begin{array}{lrr}.00 & .00 & .00 \\ .00 & 0.73 & -115.02 \\ .00 & -14.34 & .00 \\ .00 & 7.38 & .00 \\ .00 & -22.68 & .00\end{array}$

62

$436.44 \quad .00$

270.16

154.14

221.69
134.35

41.61

95.37

.00
36.09

17.07

73.67

.

21.66

1.66
.00
.00

.00
.00

.00

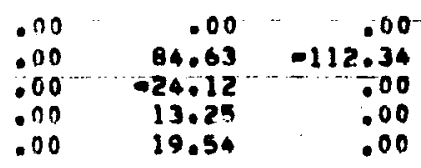

.20

97.00

$.00 \quad-25.25$

3.67

15.96
$.00 \quad .00$

10.58

28.15

$-114.04$

.00
.00
.00

D 
COCO SIMULATION

- 16.

SAMELE PROBLEM

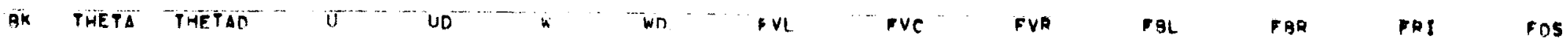

TIME $1.80000 \mathrm{LL} 1800$

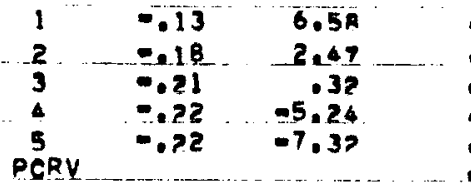

$55 \quad 17.58 \quad 2.14 \quad-5.87$

$14.18,31$

$\begin{array}{llll}1.27 & 14.51 & 2.01 & -7.69 \\ 172 & 12.44 & 2.33 & -4.75\end{array}$

\section{..}

39.73 0.60 24.90

IIME 1002000 LL 1820

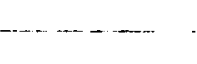

$\begin{array}{rr}.00 & 345.89 \\ 257.11 & 163.41 \\ 100.29 & 122.53 \\ 107.50 & 111.03 \\ 36.00 & 35.80\end{array}$

\begin{tabular}{|c|c|c|c|c|c|c|c|c|}
\hline $\begin{array}{l}1 \\
2 \\
3 \\
4 \\
\text { PCRV }\end{array}$ & $\begin{array}{r}103 \\
=.04 \\
=.23 \\
=.32 \\
-.34\end{array}$ & $\begin{array}{r}5.16 \\
12.20 \\
-1.30 \\
-4.69 \\
-4.17\end{array}$ & $\begin{array}{r}1.01 \\
.89 \\
.81 \\
.67 \\
.56 \\
.04\end{array}$ & $\begin{array}{r}9.55 \\
15.77 \\
18.61 \\
16.83 \\
14.89 \\
9.80\end{array}$ & $\begin{array}{l}2.00 \\
1.94 \\
1.89 \\
1.86 \\
1.88 \\
2.21\end{array}$ & $\begin{array}{l}-8.15 \\
-8.59 \\
-9.01 \\
-9.28 \\
-9.36 \\
-6.55\end{array}$ & $\begin{array}{r}124.29 \\
262.16 \\
224.67 \\
125.65 \\
10.95\end{array}$ & $\begin{array}{r}.00 \\
290.51 \\
209.40 \\
124.68 \\
41.79\end{array}$ \\
\hline
\end{tabular}

$\begin{array}{rrrrr}37.78 & .00 & 189.03 & .00 & .00 \\ 93.56 & .00 & .00 & 149.26 & -75.23 \\ 22.66 & .00 & .00 & -21.87 & .00 \\ 73.26 & .00 & .00 & -14.35 & .00 \\ 20.27 & .00 & .00 & -10.09 & .00\end{array}$

TIME $1.80000 \mathrm{LL} \quad 1840$

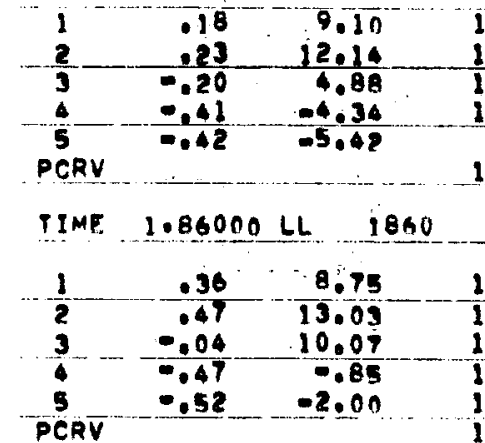

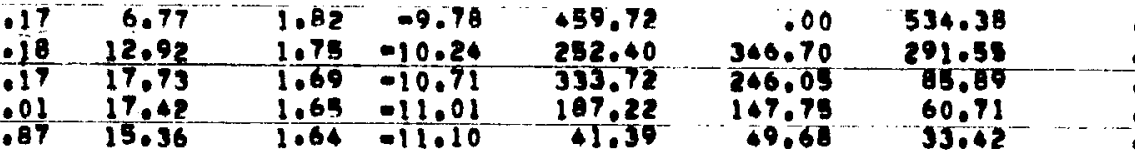
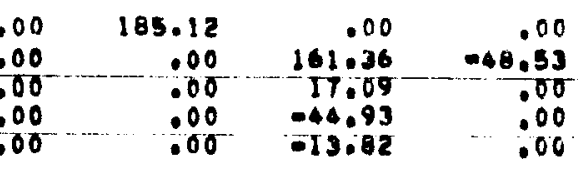

TIME $1.88000 \mathrm{LL} 1800^{\circ}$

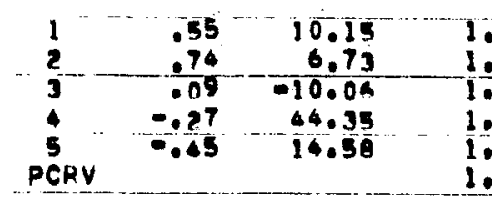

\begin{tabular}{|c|c|c|c|c|c|}
\hline $\begin{array}{lr}.28 & -1.46 \\
.55 & 5.04 \\
.77 & 4.45 \\
.71 & 13.74 \\
.93 & 18.18 \\
.22 & 0.07\end{array}$ & $\begin{array}{l}1.38 \\
1.30 \\
1.23 \\
1.19 \\
1.18 \\
1.68\end{array}$ & $\begin{array}{l}-11.80 \\
-11.95 \\
-11.66 \\
-12.21 \\
-11.31 \\
-11.21\end{array}$ & $\begin{array}{r}477.31 \\
220.05 \\
-451.65 \\
310.05 \\
123.23\end{array}$ & $\begin{array}{r}.00 \\
413.88 \\
289.63 \\
163.56 \\
62.51\end{array}$ & $\begin{array}{r}701.18 \\
427.61 \\
35.22 \\
.00 \\
21.99\end{array}$ \\
\hline
\end{tabular}

.00
.00
.00
.00
.00

269.19
151.92
1083.30
383.24
.00

.00
206.95

144.97

$-51.91$

$-35.14$

TIME $1.90000 \mathrm{LL} \quad 1900$

$\begin{array}{rrr}1 & .37 & -15.51 \\ 2 & 11 & -12.39 \\ 3 & .06 & 2.14 \\ 4 & .04 & -11.84 \\ 5 & 019 & -5.31\end{array}$

$\begin{array}{lllll}1.23 & -2.60 & 1.16 & -11.87 & 511.33 \\ 1.53 & -4.52 & 1.07 & -11.84 & 276.75 \\ 1.66 & -7.71 & .99 & -11.78 & 455.47 \\ 1.64 & -11.60 & .95 & -11.61 & 214.64 \\ 1.75 & -8.96 & .96 & -13.17 & 160.47 \\ 1.16 & -6.75 & 1.46 & -12.46 & \end{array}$

$\begin{array}{rr}.00 & 714.79 \\ 416.97 & 21.53 \\ 295.98 & 99.36 \\ 184.90 & 166.10 \\ 48.26 & 106.93\end{array}$

$\begin{array}{rr}.00 & 266.97 \\ .00 & 240.25 \\ .00 & 1171.46 \\ .00 & 1324.03 \\ .00 & 936.93\end{array}$

.00
228.49
-57.95
.55 .81
-36.56

.00
74.54
.000
.0 .00
-32.57 
COCO SIMILATION

BK THETA THETAO

TIME $1.92000 \mathrm{LL} 1920$

- 1 - $121-1.7$

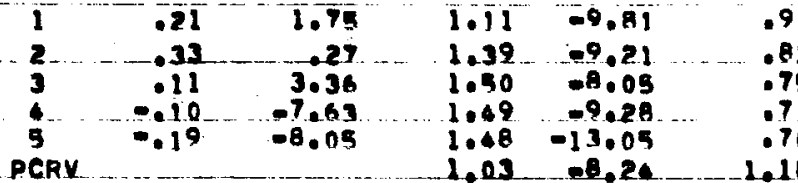

$.03=12.37$

$71-12.14$

$7 n-12.03$
$18-13.4 ?$

274.60

339.55

21 1.64

95.32

TIME $1.94000 \mathrm{LL} \quad 1940$

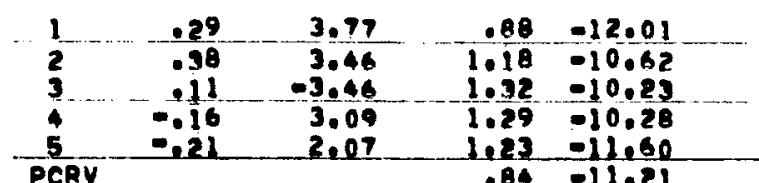

$\begin{array}{lll}.64 & -13.66 & 512.79 \\ .57 & -13.41 & 231.33 \\ .50-12.94 & 294.22 \\ .66 & -12.69 & 230.71 \\ .45 & -12.59 & 84.33\end{array}$

$\begin{array}{rr}.00 & 600.49 \\ 394.12 & 361.53 \\ 291.50 & 144.20 \\ 182.73 & 39.17 \\ 37.20 & 20.96\end{array}$

$\begin{array}{rl}.00 & 663.60 \\ 113.19 & 368.25 \\ 295.50 & 142.51 \\ 185.65 & 114.40 \\ 75.52 & 111.49\end{array}$

.00
.00
.00
.00
.00

339.64
.000
41.27
165.16
810.91

206.60

$-63.04$

$-37.76$

8.0 .05
.00

.00

TIME $1.96000 \mathrm{LL} \quad 1960^{\circ}$

\begin{tabular}{cccccccc}
1 & .33 & -1.14 & .64 & -12.52 & .36 & -14.33 & 466.63 \\
2 & .41 & -.31 & .95 & -12.50 & .29 & -14.20 & 219.97 \\
$-\frac{2}{3}$ & .05 & -1.29 & 1.09 & -12.78 & .23 & -14.12 & 310.10 \\
4 & -.05 & 7.02 & 1.08 & -11.23 & .19 & -14.07 & 210.56 \\
\hline 5 & -.08 & 0.62 & 1.01 & -10.33 & .18 & -14.06 & 61.76
\end{tabular}

TIME $1.98000 \mathrm{LL} \quad 1980$

\begin{tabular}{ccc}
1 & .28 & -1.77 \\
\hline 2 & .38 & -2.20 \\
3 & .06 & 1.17 \\
\hline 5 & .02 & -0.89 \\
\hline PCRV & .08 & -1.46 \\
\hline
\end{tabular}

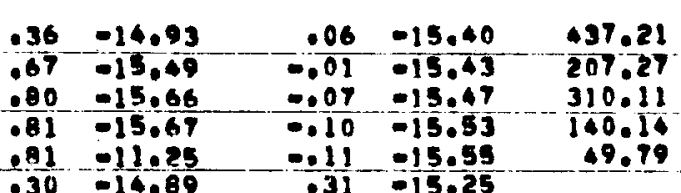

310.11

9.19

$\frac{.81}{.30}-\frac{11.25}{-14.89}-\frac{.11}{.31}-\frac{15.55}{15.25}$

TIME $2000000 \mathrm{LL} 2000$
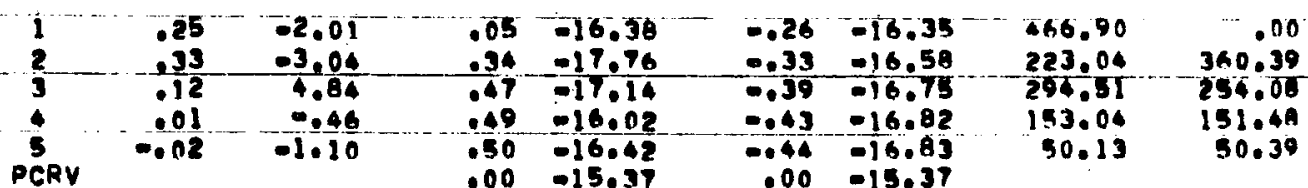

572.26
318.38
119.03
96.73
61.63

$\begin{array}{rr}.00 & 572.13 \\ 349.95 & 319.30 \\ 245.63 & 72.08 \\ 169.59 & 99.20 \\ 49.44 & 63.42\end{array}$

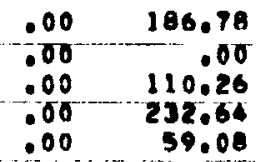

$\begin{array}{rr}.00 & .00 \\ 38.11 & 95.19 \\ 15.17 & .00 \\ 16.78 & .00 \\ 24.72 & -4.00\end{array}$

TIME 2000000 LL 2000

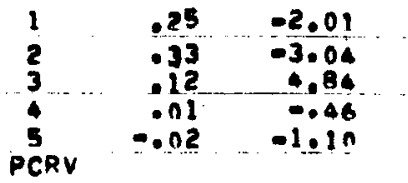

$.05-16.38$
$.34-17.76$
$.07-17.14$
$.09-16.02$
$.50-16.42$
$00-15.37$

$\begin{array}{ll}-.26 & -16.35 \quad 446.90\end{array}$

$\begin{array}{rrr}-163 & -16.82 & 153.04 \\ -.46 & -16.83 \quad 50.13\end{array}$

$.00 \quad 572.26$

$\begin{array}{ll}360.39 & 317.57 \\ 250.08 & 119.03\end{array}$

151.06

50.39

119.13
7.18

25.47

$\begin{array}{rrrr}.00 & 251.07 & .00 & .00 \\ .00 & .00 & 25.28 & 80.78 \\ .00 & 72.68 & 41.99 & .00 \\ .00 & 5.85 & 15.91 & .00 \\ .00 & .00 & -28.60 & .00\end{array}$


OUTPUT SUMMARY

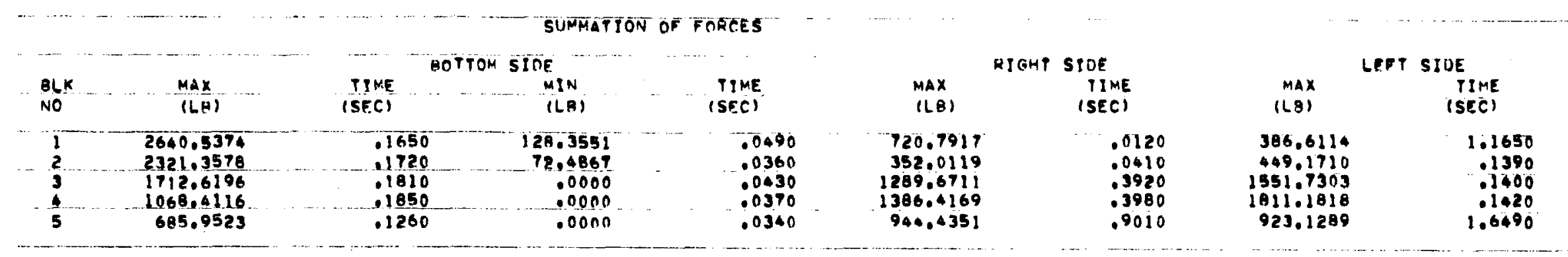

VEATICAL FORCES

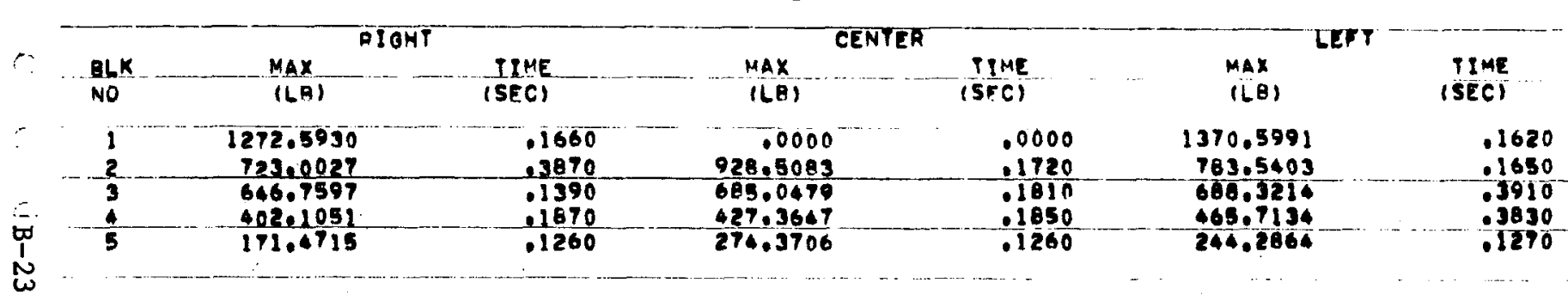




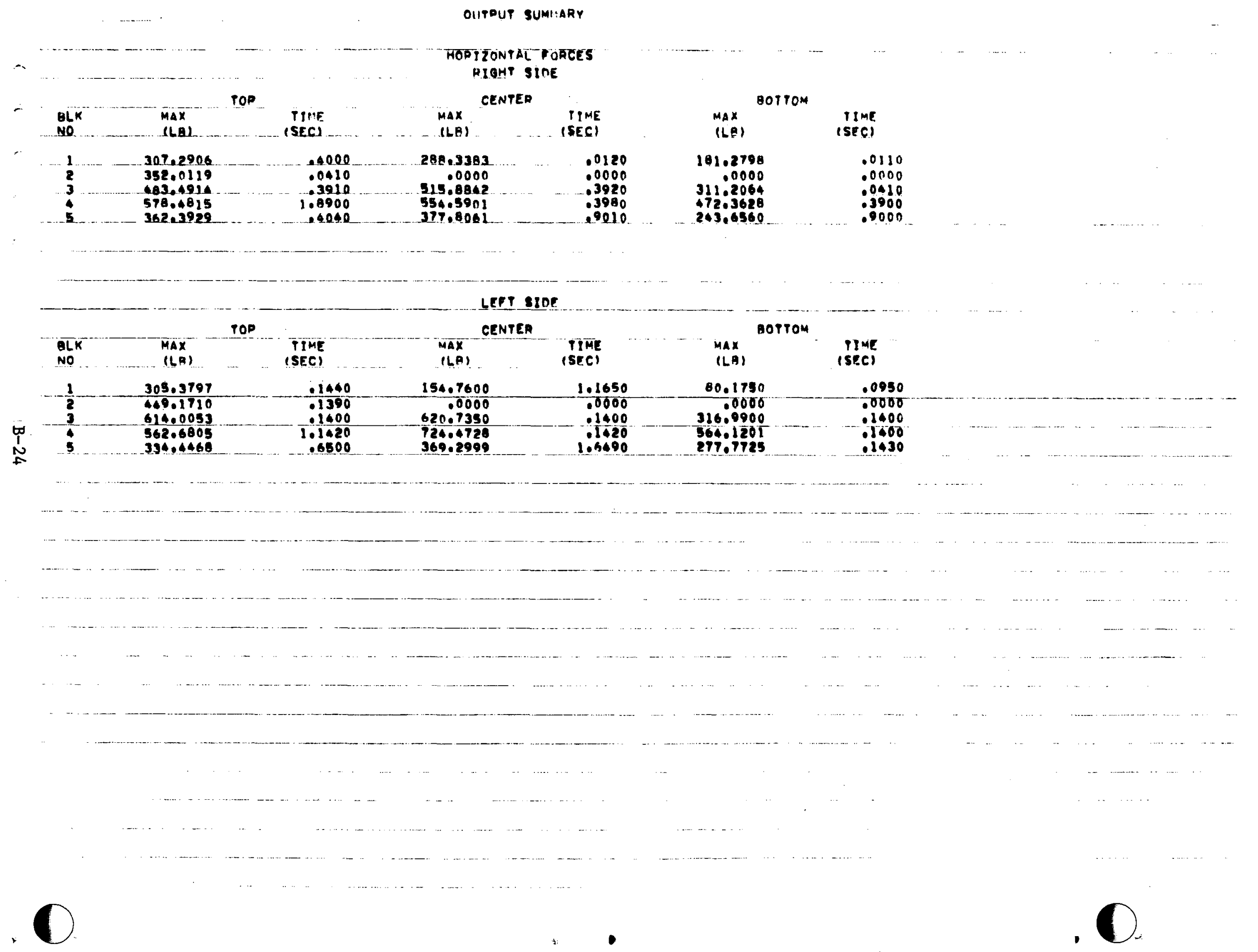


OUTPUT SUMMARY

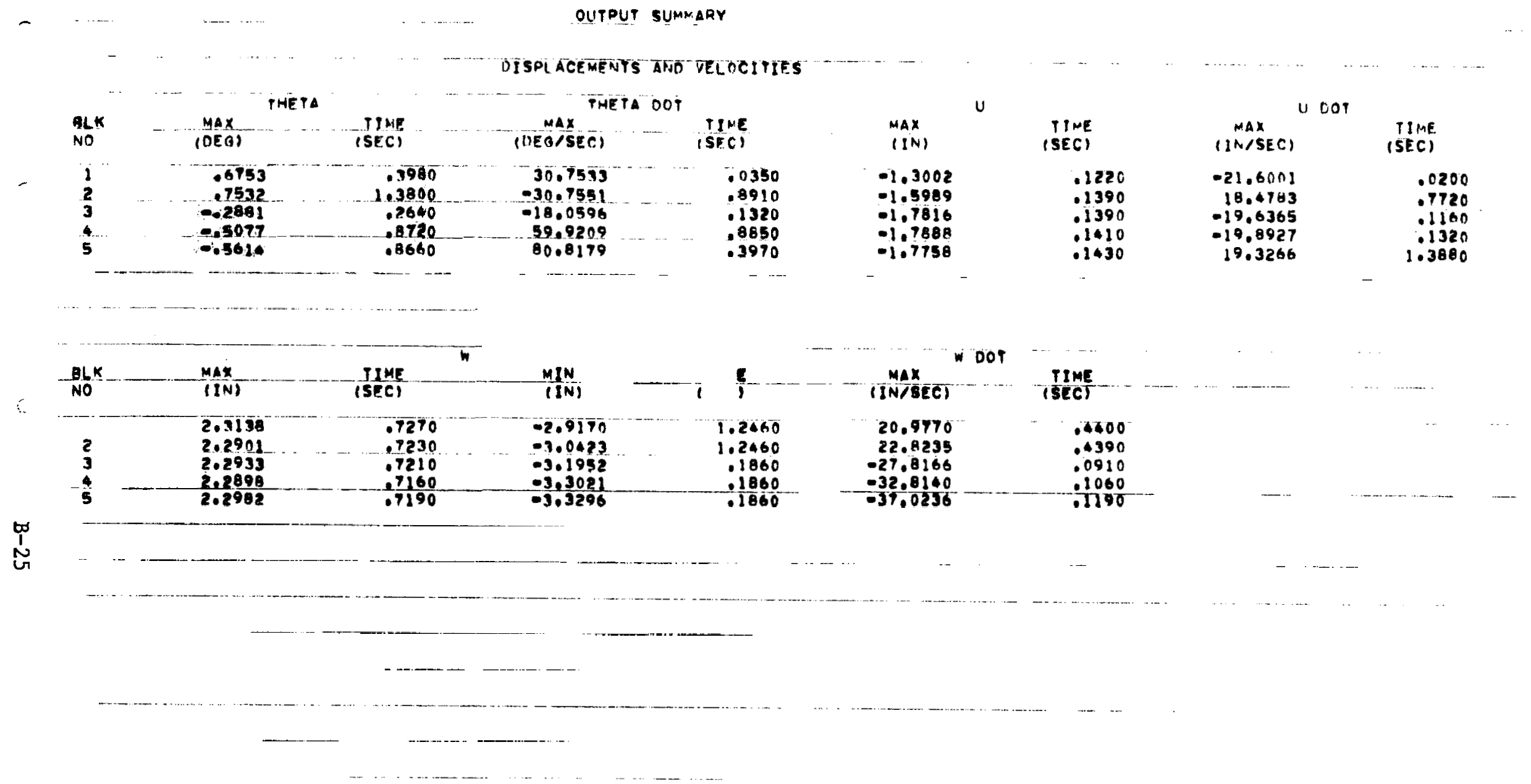


OUTPUT SUMMARY

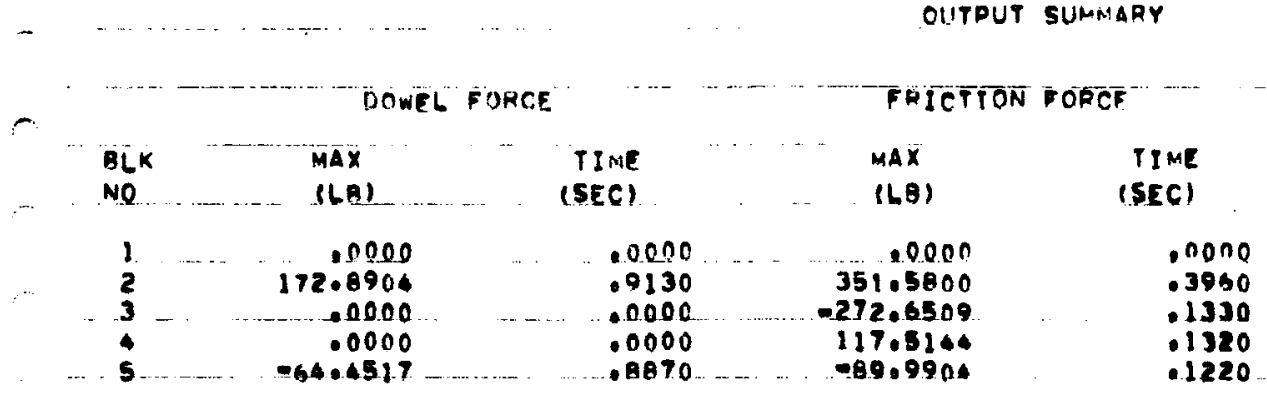

- OEXII LOCD.02z042

- F IN

Wo
1
$\alpha$ 